\title{
FASTER MIP SOLUTIONS VIA NEW NODE SELECTION RULES
}

\author{
Daniel T. Wojtaszek
}

A Thesis submitted to the Faculty of Graduate Studies and Research in partial fulfillment of the requirements for the degree of Doctor of Philosophy

May 21, 2008

Ottawa-Carleton Institute for Electrical and Computer Engineering Department of Systems and Computer Engineering

Carleton University

Ottawa, Ontario, Canada

(C) Daniel T. Wojtaszek, 2008 


$\begin{array}{ll}\begin{array}{l}\text { Library and } \\ \text { Archives Canada }\end{array} & \begin{array}{l}\text { Bibliothèque et } \\ \text { Archives Canada }\end{array} \\ \begin{array}{l}\text { Published Heritage } \\ \text { Branch }\end{array} & \begin{array}{l}\text { Direction du } \\ \text { Patrimoine de l'édition }\end{array} \\ \begin{array}{l}\text { 395 Wellington Street } \\ \text { Ottawa ON K1A 0N4 } \\ \text { Canada }\end{array} & \begin{array}{l}\text { 395, rue Wellington } \\ \text { Ottawa ON K1A 0N4 } \\ \text { Canada }\end{array}\end{array}$

Your file Votre référence

ISBN: 978-0-494-40542-0

Our file Notre référence

ISBN: 978-0-494-40542-0

NOTICE:

The author has granted a nonexclusive license allowing Library and Archives Canada to reproduce, publish, archive, preserve, conserve, communicate to the public by telecommunication or on the Internet, loan, distribute and sell theses worldwide, for commercial or noncommercial purposes, in microform, paper, electronic and/or any other formats.

The author retains copyright ownership and moral rights in this thesis. Neither the thesis nor substantial extracts from it may be printed or otherwise reproduced without the author's permission.
AVIS:

L'auteur a accordé une licence non exclusive permettant à la Bibliothèque et Archives Canada de reproduire, publier, archiver, sauvegarder, conserver, transmettre au public par télécommunication ou par l'Internet, prêter, distribuer et vendre des thèses partout dans le monde, à des fins commerciales ou autres, sur support microforme, papier, électronique et/ou autres formats.

L'auteur conserve la propriété du droit d'auteur et des droits moraux qui protège cette thèse. $\mathrm{Ni}$ la thèse ni des extraits substantiels de celle-ci ne doivent être imprimés ou autrement reproduits sans son autorisation.
In compliance with the Canadian Privacy Act some supporting forms may have been removed from this thesis.

While these forms may be included in the document page count, their removal does not represent any loss of content from the thesis.
Conformément à la loi canadienne sur la protection de la vie privée, quelques formulaires secondaires ont été enlevés de cette thèse.

Bien que ces formulaires aient inclus dans la pagination, il n'y aura aucun contenu manquant.

\section{Canada}




\section{Abstract}

When a branch and bound method is used to solve a linear mixed integer program (MIP), the order in which the nodes of the branch and bound tree are explored significantly affects how quickly the MIP is solved. When choosing a node to solve, it is desirable to either choose an ancestor of the MIP optimal node or to choose a node that is not superfluous to solving a MIP to optimality.

In this thesis, three new backtracking node selection and two backtrack triggering methods are proposed. A modification to the best-projection method is proposed that removes the requirement of finding an incumbent solution in order for this method to be used. This first proposed method is used as both a backtracking node selection method and as a method of backtrack triggering. A second proposed backtracking node selection method balances the goals of MIP feasibility and MIP optimality by using probability distributions to model these measures based on all nodes solved thus far. A third proposed backtracking node selection method determines when the cost of using a backtracking node selection method outweighs its benefit, in which case it is abandoned in favor of simple backtracking. Finally, a proposed backtrack triggering method estimates the MIP optimal objective value using the objective values of nodes at the estimated depth of a MIP optimal node.

Empirical results show that these proposed methods outperform the state of the art by a large margin with respect to speeding up the solution process of many MIP instances. 


\section{Acknowledgements}

I would like to thank my supervisor Dr. John Chinneck for giving me this opportunity to work with him. I benefited greatly from his guidance and I will continue to do so for the rest of my academic and professional career.

I would like to thank my parents and my sister for their support and encouragement. 


\section{Contents}

$\begin{array}{ll}\text { Abstract } & \text { ii }\end{array}$

Acknowledgements

List of Tables vii

List of Figures $\quad$ xi

List of Abbreviations $\quad$ xv

List of Symbols $\quad$ xvii

1 Introduction 1

2 A Review of Mixed Integer Programming 10

2.1 Definition of Mixed Integer Program . . . . . . . . . . 10

2.2 The Branch and Bound Method . . . . . . . . . . . . . 11

2.2 .1 Branching Variable Selection . . . . . . . . . . . . . 14

2.2 .2 Node Selection . . . . . . . . . . . . . . . 15

3 State of the Art in Heuristic Node Selection Methods $\quad 17$

3.1 Basic Node Selection Methods . . . . . . . . . . . . . . 17

3.2 Estimate based Node Selection . . . . . . . . . . . . . . . . . 19

3.2.1 Best-Projection Node Selection . . . . . . . . . . . 19 
3.2.2 Best-Estimate Node Selection . . . . . . . . . . . . . 20

3.3 Genetic Programming and Node Selection . . . . . . . . . . 22

3.4 Triggering Backtracking $\ldots \ldots \ldots \ldots \ldots \ldots$

3.5 Solver Specific Heuristic Node Selection Methods . . . . . . . . . 25

3.6 State of the Art in MIP Software . . . . . . . . . . . . 27

4 The Node Selection Problem 29

4.1 Performance of the State of the Art . . . . . . . . . . . . 29

4.1.1 Backtracking Node Selection . . . . . . . . . . . . . . 29

$4.1 .2 \quad$ Backtrack Triggering . . . . . . . . . . . . . . 30

5 New Methods for Backtrack Triggering and Node Selection 33

5.1 Modified Best Projection Node Selection and Aspiration Method . . . 34

5.1 .1 Algorithm . . . . . . . . . . . . . . . 37

5.2 Distribution Node Selection $\ldots \ldots \ldots \ldots$. . . . . . 41

5.2 .1 Algorithm . . . . . . . . . . . . . . . . . 44

5.3 Feasibility Depth Extrapolation Aspiration . . . . . . . . . . 46

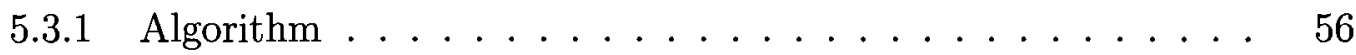

5.4 Active Node Search Threshold . . . . . . . . . . . . . . 58

5.4 .1 Algorithm . . . . . . . . . . . . . . . 62

6 Experimental Setup $\quad 65$

6.1 Hardware . . . . . . . . . . . . . . . . 65

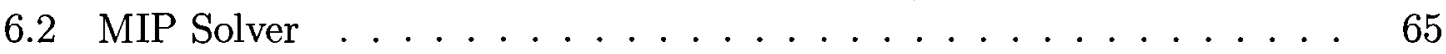

6.3 Performance Measures . . . . . . . . . . . . . . . 66

6.4 MIP Instances . . . . . . . . . . . . . . . . . . . . . 71

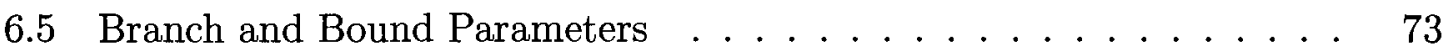

6.5.1 Branching Variable Selection Parameter . . . . . . . . 73

6.5.2 Root Node Cuts . . . . . . . . . . . . . . . . . 75

6.5.3 Node Selection Parameters . . . . . . . . . . . . . 76 
7 Experimental Results $\quad 77$

7.1 Identification of Promising Configurations . . . . . . . . . 81

7.2 Testing of Promising Configurations . . . . . . . . . . . . . . 95

7.3 Problem-specific Node Selection . . . . . . . . . . . . . 102

8 Conclusions $\quad 106$

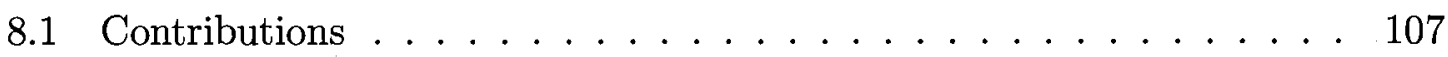

8.2 Future Research . . . . . . . . . . . . . . . 108

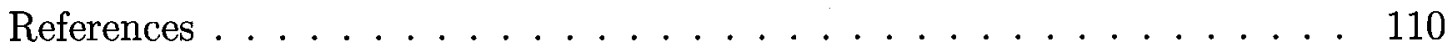

A MIP Instances $\quad 114$

B Experimental Data $\quad 126$

B.1 Branching Variable Selection Data . . . . . . . . . . . 126

B.2 Cuts Data . . . . . . . . . . . . . . . . . . 127

B.3 Identification of Promising Configurations Data . . . . . . . . . . 127

B.4 Testing of Promising Configurations Data . . . . . . . . . . 127

B.5 Proof of Concept: Perfect Aspiration Value Data . . . . . . . . . . 128 


\section{List of Tables}

3.1 MIP solver software and available standard backtracking node selection methods. . . . . . . . . . . . . . . . . .

4.1 Performance of perfect aspiration value versus the state of the art methods. The best values in each of the TOTTM and MRATE columns are in boldface. (see Page 68 for the definition of $F A I L$ ) . . . . . .

5.1 Comparing the solution time, number of nodes, and number of simplex iterations using best-projection (DEBP-NONA-NOAN) vs. depth-first (DEPF-NONA-NOAN) node selection. Also shown is $R_{t}$. The best values with respect to time in each of the rows are in bold as are the best values with respect to itns and nodes. . . . . . . . . . .

6.1 The minimum, average, and maximum values of the given formulation

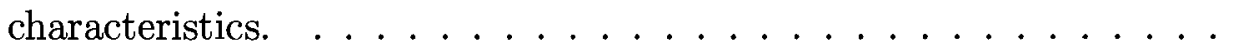

6.2 Performance of contending branching variable selection methods. The best values in each of the FAIL, TOTTM and MRATE columns are in bold. . . . . . . . . . . . . . . . . . .

6.3 Performance of root node cuts vs no cuts. The best values in each of the FAIL, TOTTM and MRATE columns are in bold. . . . . . . . .

7.1 List of all competitor node selection configurations. The configurations that are available in the original GLPK solver are in boldface. . . . . 
7.2 Performance of each configuration in Table 7.1 with respect to MRATE. Also shown is the variance of $r_{M I}$ over all MIP instances for each configuration. The configurations that are available in the original GLPK solver are in boldface. . . . . . . . . . . . . . . . . . .

7.3 The p-values of the analysis of variance (ANOVA) tests of MRATE between the best ranked state of the art configuration DEBP-DBPANOAN and each of the top seven ranked configurations. Smaller pvalues mean that the difference between the values of $M R A T E$ for considered methods is more significant. . . . . . . . . . . . . . .

7.4 Performance of each configuration in Table 7.1 with respect to TOTTM. The configurations that are available in the original GLPK solver are in boldface. . . . . . . . . . . . . . . . . .

7.5 Performance of each configuration in Table 7.1 and their rankings. The configurations that are available in the original GLPK solver are in boldface. The smallest values of $F A I L, T R$, and $R R$ are also in boldface. . . . . . . . . . . . . . . . .

7.6 Average performance of configurations with NOAN vs ANST. Configurations with BREF and DEPF are excluded. The best values of $F A I L$, TOTTM, and MRATE are in boldface. . . . . . . . . . .

7.7 Average performance of each backtrack triggering method. DEPFNONA-NOAN is excluded. The best values of FAIL, TOTTM, and $M R A T E$ are in boldface. . . . . . . . . . . . . .

7.8 Rank of configurations with the same backtracking node selection option. 92

7.9 List of promising node selection configurations for the stage 2 experiment along with their rank $\mathrm{R}$ determined in Section 7.1 and why they are included in this experiment. . . . . . . . . . . . 
7.10 The Performance of each configuration in Table 7.9: (a) over all MIP instances; (b) excluding MIP instances used in the experiment in Section 7.1. The smallest values of FAIL, TOTTM, MRATE, AVGRANK, and NINC are in boldface. The largest value of NFIRSTS is also in boldface. Also shown is the variance of $M R A T E, M V A R$, and the variance of $A V G R A N K, R V A R . \ldots \ldots \ldots \ldots$

7.11 The p-values of the ANOVA tests of MRATE between the best state of the art configuration and each of the proposed configurations. . . .

7.12 The performance of two state of the art configurations versus the MOBP-MPAS-ANST configuration with respect to time for solving FCTPs. Each FCTP is solved by at least one of these configurations. Solved $=1$ if a configuration solves a model within the time limit; Solved $=0$ if a configuration does not solve a model within the time limit. Diff time is the difference between the time to solve a model using MOBP-MPAS-ANST and the smaller of the times to solve this model using either of the state of the art configurations. . . . . . . 104

7.13 The performance of two state of the art configurations versus the DISTALLT-NOAN configuration with respect to the best incumbent solution found for FCTPs that were not solved within the time limit. $r z_{i n c}$ is the ratio of the best incumbent solution found using DIST-ALLTNOAN and the smallest of incumbent solutions found using either of the state of the art configurations. . . . . . . . . . . 105

A.1 List of MIP instances taken from MIPLIB 2003, MIPLIB 3.0, MIPLIB 2.0 and CORAL with primal LP and MIP formulation characteristics. 115

A.2 List of MIP instances taken from MIPLIB 2003, MIPLIB 3.0 and MIPLIB 2.0. These MIP instances are used in the experiments described in Sections 4.1.2, 6.5.1, and 6.5.2. . . . . . . . . . . . . 
A.3 List of MIP instances in Table A.1 which are solved within 30 minutes for the experiment in Section 7.1 using the default GLPK node selection

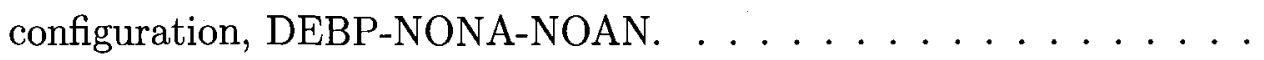

A.4 List of MIP instances in Table A.1 which are solved within 60 minutes for the experiment in Section 7.2 using at least one of the node selection configurations listed in Table 7.9. . . . . . . . . . . . . . . . . 123

A.5 List of MIP instances in Table A.1 which are not solved within 60 minutes for the experiment in Section 7.2 using any of the node selection configurations listed in Table 7.9. MIP instances for which no incumbent solution was found using any of these configurations are excluded. . . . . . . . . . . . . . . . . . . . 12

A.6 List of MIP instances in Table A.1 which are not solved and no incumbent solution was found within 60 minutes for the experiment in Section 7.2 using any of the node selection configurations listed in Table $7.9 \ldots \ldots \ldots \ldots \ldots \ldots \ldots$ 


\section{List of Figures}

1.1 Branch and bound tree of MIPEX when it is solved using $D F-B F-$ $A S P=11 \ldots \ldots \ldots \ldots \ldots \ldots \ldots \ldots \ldots \ldots \ldots$

1.2 Branch and bound tree of MIPEX when it is solved using $D F-D F . \quad 7$

4.1 A performance profile comparing the NONA, PERA, DBPA, and PCAS options for aspiration method. . . . . . . . . . . .

5.1 Plot of $z_{i}$ and $z^{m i n}(c)$ versus $c_{i}$ for all of the nodes in the tree at the completion of the branch and bound process for the dcmulti MIP instance. Also shown is the best fit line through $z^{\min }(c)$ for $10 \leq c \leq$ 30. The DEBP-NONA-NOAN node selection configuration is used (see Chapter 7 for the configuration definitions). . . . . . . . .

5.2 Plot of $z_{i}$ and $z^{m i n}(c)$ versus $c_{i}$ for all of the nodes in the tree at the completion of the branch and bound process for the fixnet/4 MIP instance. Also shown is the best fit line through $z^{\min }(c)$ for $5 \leq c \leq 15$. The DEBP-NONA-NOAN node selection configuration is used. . . .

5.3 Plot of $z_{i}$ and $z^{\min }(c)$ versus $c_{i}$ for all of the nodes in the tree at the time in the branch and bound process when $c_{\min }=23$ for the dcmulti MIP instance. Most of the points in the plot overlap because $z_{i}=z^{\min }(c)$ for most of the nodes at this time. Also shown is the line through $z^{m i n}(55)$ (the root node) and $z^{m i n}(23) \ldots \ldots \ldots \ldots$ 
5.4 A plot of the objective value versus depth for all nodes for the ran10x10a MIP instance. Also shown are lines representing the MIP optimal objective value and depth. The DIST-NONA-NOAN node selection configuration is used. . . . . . . . . . . . . .

5.5 A plot of the ratio of depth of a MIP optimal node to the minimum depth over all MIP feasible nodes for a set of MIP instances. The DEBP-NONA-NOAN node selection configuration is used. . . . . . .

5.6 A plot of the number of candidate variables versus depth for all ancestors of a MIP optimal node for the 10teams MIP instance. . . . . . .

5.7 A plot of the number of candidate variables versus depth for all ancestors of a MIP optimal node for the bell5 MIP instance. . . . . . . . .

5.8 A plot of the number of candidate variables versus depth for all ancestors of a MIP optimal node for the vpm2 MIP instance. . . . . . . .

5.9 A plot of the number of candidate variables versus depth for the earliest 20 ancestors of a MIP optimal node for the 10teams MIP instance along with the best fit line. . . . . . . . . . . . . . . . . . .

5.10 A plot of the number of candidate variables versus depth for the earliest 20 ancestors of a MIP optimal node for the bell5 MIP instance along with the best fit line. . . . . . . . . . . . . . . . . . .

5.11 A plot of the number of candidate variables versus depth for the earliest 20 ancestors of a MIP optimal node for the vpm2 MIP instance along with the best fit line. . . . . . . . . . . . . . . . .

7.1 A plot showing MRATE, $r_{p 10}$, and $r_{p 90}$ for each configuration. $r_{p 10}$ and $r_{p 90}$ are the 10th and 90th percentiles, respectively, of $r_{M I}$ (see Page 67 for definition) over all MIP instances for a given configuration. Each $\mathrm{x}$-axis value of $R R$ uniquely corresponds to a configuration as is shown in Table $7.2 \ldots \ldots \ldots \ldots \ldots \ldots \ldots \ldots$ 
7.2 A plot showing the average, minimum, and maximum value of $F A I L$ for each of the following backtrack triggering methods: method $1=$ NONA, method $2=$ ALLT, method $3=$ DBPA, method $4=$ PCAS,

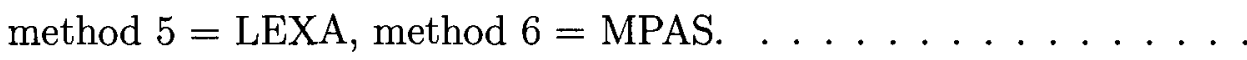

7.3 A plot showing the average, minimum, and maximum value of TOTTM for each of the following backtrack triggering methods: method $1=$ NONA, method $2=$ ALLT, method $3=$ DBPA, method $4=$ PCAS, method $5=$ LEXA, method $6=$ MPAS. . . . . . . . . .

7.4 A plot showing the average, minimum, and maximum value of MRATE for each of the following backtrack triggering methods: method $1=$ NONA, method $2=$ ALLT, method $3=$ DBPA, method $4=$ PCAS, method $5=$ LEXA, method $6=$ MPAS. . . . . . . . . . .

7.5 A plot showing the average, minimum, and maximum ranking for each of the backtracking node selection methods shown in Table 7.8; method 1: MOBP, method 2: DIST, method 3: BFBE, method 4: DEBP, method 5: BEES, method 6: BESF, method 7: BREF. . . . . . . .

7.6 A plot showing the average, minimum, and maximum value of $F A I L$ for each of the backtracking node selection methods shown in Table 7.8; method 1: MOBP, method 2: DIST, method 3: BFBE, method 4: DEBP, method 5: BEES, method 6: BESF, method 7: BREF. . . . .

7.7 A plot showing the average, minimum, and maximum value of TOTTM for each of the backtracking node selection methods shown in Table 7.8; method 1: MOBP, method 2: DIST, method 3: BFBE, method 4: DEBP, method 5: BEES, method 6: BESF, method 7: BREF. . . . .

7.8 A plot showing the average, minimum, and maximum value of $M R A T E$ for each of the backtracking node selection methods shown in Table 7.8; method 1: MOBP, method 2: DIST, method 3: BFBE, method 4: DEBP, method 5: BEES, method 6: BESF, method 7: BREF. . . . . 
7.9 A plot showing MRATE, $r_{p 10}$, and $r_{p 90}$ for the following configurations: method $1=$ MOBP-MPAS-ANST, method $2=$ MOBP-PCASANST, method $3=$ DIST-MPAS-ANST, method $4=$ MOBP-LEXAANST, method 5: DIST-ALLT-NOAN, method 6: MOBP-MPASNOAN, method 7: MOBP-PCAS-NOAN, method 8: DEBP-DBPANOAN, method 9: DEBP-NONA-NOAN. . . . . . . . . . . . 100

7.10 The performance profile comparing the two best proposed configurations with the two state of the art methods. Each point in the plot represents the fraction of MIP instances for which the ratio of time to solve them using the given configuration to best time to solve them over all the configurations in Table 7.9 is less than or equal to its value at that point. . . . . . . . . . . . . . . . . . . 101 


\section{List of Abbreviations}

ALLT Trigger backtracking after every node solution................. 78

ANST Active Node Search Threshold $\ldots \ldots \ldots \ldots \ldots \ldots \ldots \ldots \ldots \ldots \ldots, 8$

BDRTO Branching variable selection method by Driebeek [14] and Tomlin [38] 73

BEES Best-estimate node selection $\ldots \ldots \ldots \ldots \ldots \ldots \ldots \ldots \ldots \ldots \ldots \ldots, 77$

BESF Best-first node selection $\ldots \ldots \ldots \ldots \ldots \ldots \ldots \ldots \ldots \ldots \ldots \ldots \ldots, 77$

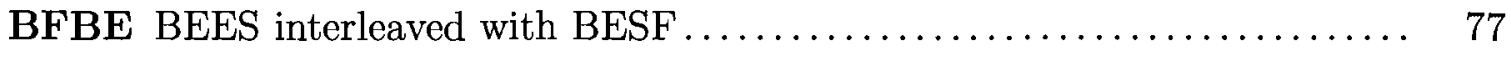

BFIRST Branch on the first candidate variable .................... 73

BLAST Branch on the last candidate variable ...................... 73

BMFRAC Branch on the most fractional candidate variable............ 73

BREF Breadth-first node selection $\ldots \ldots \ldots \ldots \ldots \ldots \ldots \ldots \ldots \ldots \ldots \ldots, 77$

CUTS root node cuts................................... 75

DBPA Default best-projection aspiration $\ldots \ldots \ldots \ldots \ldots \ldots \ldots \ldots \ldots \ldots \ldots, 78$

DEBP Default best-projection node selection $\ldots \ldots \ldots \ldots \ldots \ldots \ldots \ldots \ldots, 77$

DEPF Depth-first node selection............................. 77

DIST Distribution node selection $\ldots \ldots \ldots \ldots \ldots \ldots \ldots \ldots \ldots \ldots \ldots \ldots, 78$ 
LEXA Linear feasibility depth extrapolation aspiration $\ldots \ldots \ldots \ldots \ldots \ldots \ldots$

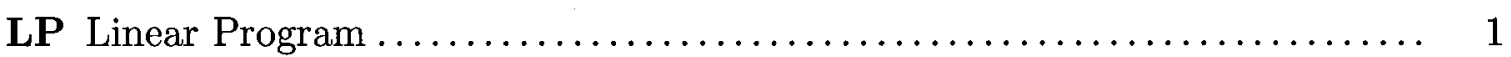

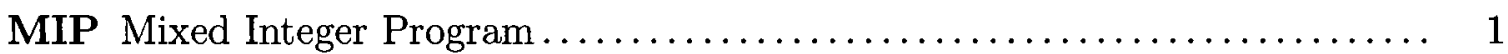

MOBP Modified best-projection node selection $\ldots \ldots \ldots \ldots \ldots \ldots \ldots \ldots \ldots$

MPAS Modified best-projection aspiration..................... 78

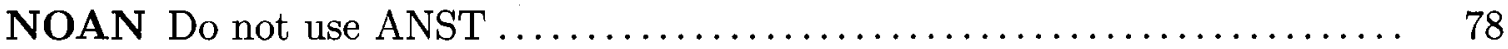

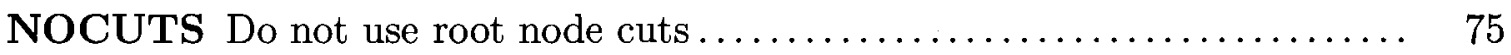

NONA Non-aspiration backtack triggering ..................... 78

PCAS Pseudo-cost (best-estimate) aspiration $\ldots \ldots \ldots \ldots \ldots \ldots \ldots \ldots \ldots$

PERA Use optimal objective value to trigger backtracking $\ldots \ldots \ldots \ldots \ldots \ldots .31$ 


\section{List of Symbols}

$z \quad$ The objective value of an $L P \ldots \ldots \ldots \ldots \ldots \ldots \ldots \ldots \ldots \ldots \ldots \ldots \ldots \ldots, \quad 3$

$z_{i n c}$ The objective value of an incumbent solution $\ldots \ldots \ldots \ldots \ldots \ldots \ldots \ldots$

$z^{*} \quad$ The MIP optimal objective value............................. 4

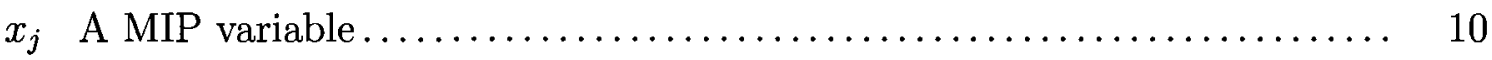

$L_{u} \quad$ The list of unexplored nodes............................... 13

$s_{i}$ The sum of infeasibilities................................. 18

$f_{j}$ The fractional part of a variable $\ldots \ldots \ldots \ldots \ldots \ldots \ldots \ldots \ldots \ldots \ldots \ldots \ldots \ldots \ldots$

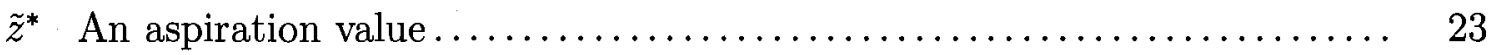

$c$ The number of candidate variables $\ldots \ldots \ldots \ldots \ldots \ldots \ldots \ldots \ldots \ldots \ldots, 34$

$z^{\text {min }}(c)$ The minimum objective over all nodes with $c$ candidate variables... 34

$c_{\text {min }}$ The minimum number of candidate variables over all nodes $\ldots \ldots \ldots \ldots . \quad 37$

$\sigma_{z}$ The standard deviation of $z \ldots \ldots \ldots \ldots \ldots \ldots \ldots \ldots \ldots \ldots \ldots \ldots \ldots \ldots \ldots$

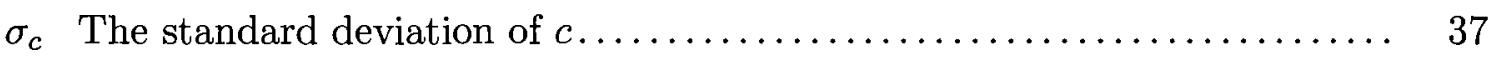

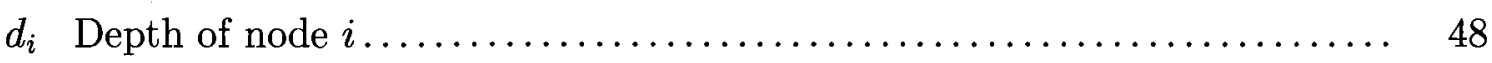

$\tilde{d}^{*} \quad$ An estimate of depth of an optimal node..................... 53 
$r_{M I}($ time to solve a MIP $M I) /($ best time to solve $M I) \ldots \ldots \ldots \ldots \ldots \ldots$. 67

$F A I L \quad$ The number of unsolved MIP instances $\ldots \ldots \ldots \ldots \ldots \ldots \ldots \ldots \ldots$

TOTTM The total time to solve a set of MIP instances ............. 68

MRATE The mean ratio to best time to solve a set of MIP instances..... 69

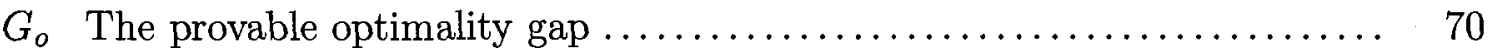

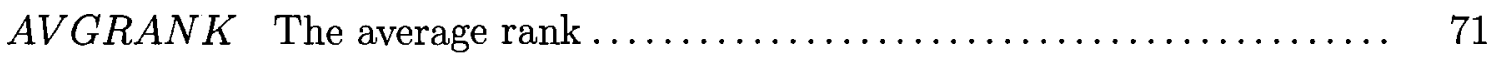

NFIRSTS The number of times that a method is ranked first.......... 71

NINC The number of times that no incumbent solution is found........ 71

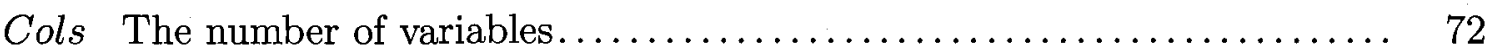

Real The number of real variables $\ldots \ldots \ldots \ldots \ldots \ldots \ldots \ldots \ldots \ldots \ldots \ldots \ldots \ldots \ldots \ldots$

Int The number of non-binary integer variables................... 72

Bin The number of binary variables $\ldots \ldots \ldots \ldots \ldots \ldots \ldots \ldots \ldots \ldots \ldots \ldots \ldots \ldots \ldots \ldots$

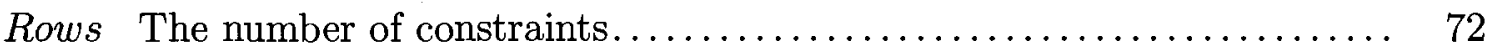

Ineq The number of inequality constraints $\ldots \ldots \ldots \ldots \ldots \ldots \ldots \ldots \ldots \ldots \ldots$

$E q$ The number of equality constraints........................ 72

$N Z$ The number of non-zero constraint and objective function coefficients... 72

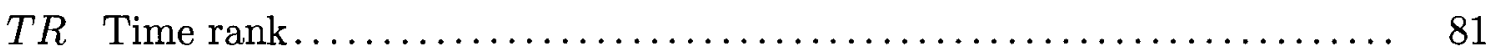

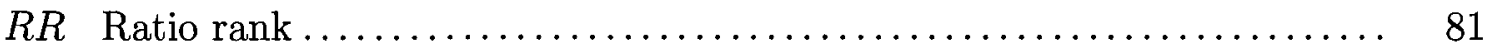

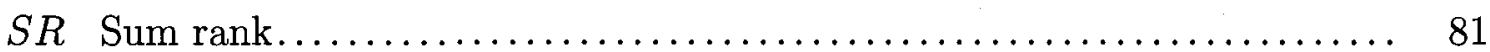

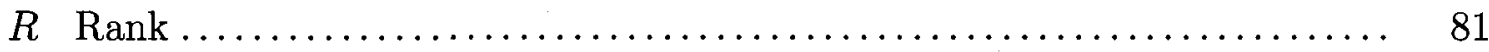

xviii 


\section{Chapter 1}

\section{Introduction}

Linear Mixed Integer Programs (MIPs) are used to model many problems which involve optimization such as spacecraft trajectory planning [36], hardware/software codesign [31], and scheduling [23].

A MIP, described in Section 2.1, is a Linear Program ( $L P)$ in which one or more of the variables are constrained to be integer valued. The most commonly used method of solving MIPs to proven optimality is called branch and bound, described in Section 2.2. A difficulty with branch and bound is that it can take an impractical amount of time to solve many MIPs even when using the most sophisticated computers.

The chosen node selection method is one aspect of branch and bound that greatly affects how quickly it can solve MIPs by determining the manner in which a branch and bound tree is explored.

To demonstrate how node selection can affect the performance of the branch and bound method, the following MIP instance MIPEX is solved using the branch and bound method with different node selection methods and the resulting branch and bound trees are shown. 


\begin{tabular}{ll} 
& $M I P E X$ \\
\hline $\min$ & $z=x+3 y$ \\
st. & $4 x+5 y \leq 41$ \\
& $13 x-8 y \geq 12$ \\
& $7 x+33 y \geq 96$ \\
& $0 \leq x \leq 10$ \\
& $0 \leq y \leq 10$ \\
& $x, y \in \mathbb{Z}$
\end{tabular}

A solution of MIPEX is a point $(x, y)$ that: yields the minimum value of $z$, satisfies all of the linear constraints, and has integer values for both $x$ and $y$. The branch and bound method starts by solving $M I P E X$ without the integer constraints on $x$ and $y$, called the LP-relaxation. MIPEX without the integer constraints is the root node of the branch and bound tree. The solution of the root node is

$$
\begin{aligned}
& z=9.6 \\
& x=2.4 \\
& y=2.4
\end{aligned}
$$

This solution is LP feasible since all of the linear constraints are satisfied. The value of $y$ in the solution to the root node is fractional so two child nodes are created by changing the bounds on $y$ so that its value is forced away from this fractional value. In one child node, the upper bound on the value of $y$ is changed to $0 \leq y \leq 2$, called the down branch. In the other child node, the lower bound on the value of $y$ is changed to $3 \leq y \leq 10$, called the up branch. In this case, $y$ is the branching variable. It should be noted that $x$ can be chosen as the branching variable instead of $y$ and that this will result in a different branch and bound tree. At this point a node selection method is employed to choose which of these child nodes will be solved next. If the solution of a node is LP feasible and has integer values for both $x$ and $y$ then this node is MIP feasible. The process of solving a node, choosing 
a branching variable, creating child nodes, and selecting the next unsolved node to solve is repeated until (i) a MIP feasible node is found that has an objective value $z$ that is less than or equal to the value of $z$ for all of the nodes that have at least one unsolved child node, or (ii) there are no unsolved nodes left in the tree. The MIP feasible node with the smallest objective value over all MIP feasible nodes is the MIP optimum. The optimal solution of $M I P E X$ is

$$
\begin{aligned}
& z=11 \\
& x=5 \\
& y=2 .
\end{aligned}
$$

There are many possible node selection methods that can be used to solve MIPEX, each of which may result in a different branch and bound tree. Two possible node selection methods that can be used to solve MIPEX are described below. These methods are chosen arbitrarily to demonstrate how using different node selection methods can affect the branch and bound tree.

$D F-B F-A S P=11:$ Depth-first with best-first backtracking node selection and aspiration value of 11 chooses the next node to solve according to the following rules. If the most recently solved node is LP feasible but not MIP feasible, then choose a child of this node if $z \leq 11$ for this node. Backtracking occurs if the most recently solved node

- is not LP feasible, or

- is MIP feasible, or

- has an objective value that is greater than or equal to the smallest objective value $z_{i n c}$ over all MIP feasible solutions found so far, or

- has an LP-relaxation objective value that is greater than 11 .

Best-first backtracking chooses a child of the node that has the smallest value of $z$. 
$D F-D F:$ Depth-first with depth-first backtracking node selection chooses the next node to solve according to the following rules. If the most recently solved node is LP feasible but not MIP feasible, then choose a child of this node. If the most recently solved node is a leaf node, meaning that it is either MIP feasible, not LP feasible, or has a value of $z \geq z_{i n c}$, then backtrack by choosing a child of the most recently solved LP feasible node.

If an LP feasible node has two unsolved child nodes then choose the up branch. For example, the root node child that corresponds with the bounds on $y$ being $3 \leq y \leq 10$ is solved before its sibling.

The aspiration value used in $D F-B F-A S P=11$ is based on an a priori estimate of the MIP optimal objective value $z^{*}$ to demonstrate how such knowledge can be used to avoid the exploration of superfluous nodes. Superfluous nodes are nodes for which $z>z^{*}$. It is not necessary to explore these nodes in order to prove that a MIP feasible solution is optimal. Knowledge of the value of $z^{*}$ for a given MIP instance is usually not available until the MIP instance has been solved, so its value must be estimated in order for it to be used to avoid the exploration of superfluous nodes.

Figures 1.1 and 1.2 show the solved nodes in the branch and bound trees when using the $D F-B F-A S P=11$ and the $D F-D F$ node selection methods respectively to solve MIPEX, along with the order in which each node is solved.

During the branch and bound process of solving $M I P E X$ using $D F-B F-$ $A S P=11$, the second node that is solved has a value of $z>11$ so backtracking is triggered. Best-first backtracking then chooses node 2 which is an ancestor of the MIP optimal node. Depth-first node selection then proceeds to choose node3, the remaining ancestor of the MIP optimal node, followed by node4, the MIP optimal node. At this point node 2 and node 3 both have an unsolved child with $z<z_{\text {inc }}$ that are each chosen in turn using best-first backtracking.

During the branch and bound process of solving $M I P E X$ using $D F-D F$, the third node that is solved, node2, is MIP feasible so this method requires less effort to 
find a MIP feasible solution than $D F-B F-A S P=11$, which solves 5 nodes before finding a MIP feasible solution. Depth-first backtracking then chooses node3 to be solved next. Node3 is a leaf, so depth-first backtracking then chooses node4 which is an ancestor of the MIP optimal node. Depth-first node selection then proceeds to choose node5, the remaining ancestor of the MIP optimal node, followed by node6, the MIP optimal node. At this point node 5 and node4 both have an unsolved child which are each chosen in turn using depth-first backtracking.

In this example, solving $M I P E X$ using $D F-B F-A S P=11$ resulted in over $25 \%$ fewer solved nodes than when using $D F-D F$. Solving $M I P E X$ using $D F-D F$, on the other hand, resulted in a MIP feasible solution being found in over $65 \%$ fewer solved nodes. Therefore, if the goal is to find a MIP feasible solution of MIPEX as quickly as possible, then $D F-D F$ should be used rather than $D F-B F-A S P=11$. If the goal is to solve MIPEX as quickly as possible, then $D F-B F-A S P=11$ should be used rather then $D F-D F$.

In order to quickly solve a MIP, node selection methods try to order the exploration of nodes in such a way that the MIP optimal node is quickly found by exploring the ancestors of the MIP optimal node before all of the other nodes. Whether a node is an ancestor of a MIP optimal node is generally unknown, therefore node selection methods employ heuristic rules in order to quickly find a MIP optimal solution. A commonly used framework for node selection is depth-first with backtracking. This framework uses depth-first node selection except when it is determined that backtracking is necessary and a different node selection method is employed. Two aspects of depth-first with backtracking are (i) the backtracking node selection and (ii) backtrack triggering methods. Backtracking node selection methods rank all available nodes according to some criteria and choose the next node to solve accordingly. Backtrack triggering methods classify the most recently solved node as either desirable or undesirable. If this node is undesirable then a backtracking node selection method is used to choose the next node to solve. Backtracking node selection is also used if the most recently solved node is a leaf. 


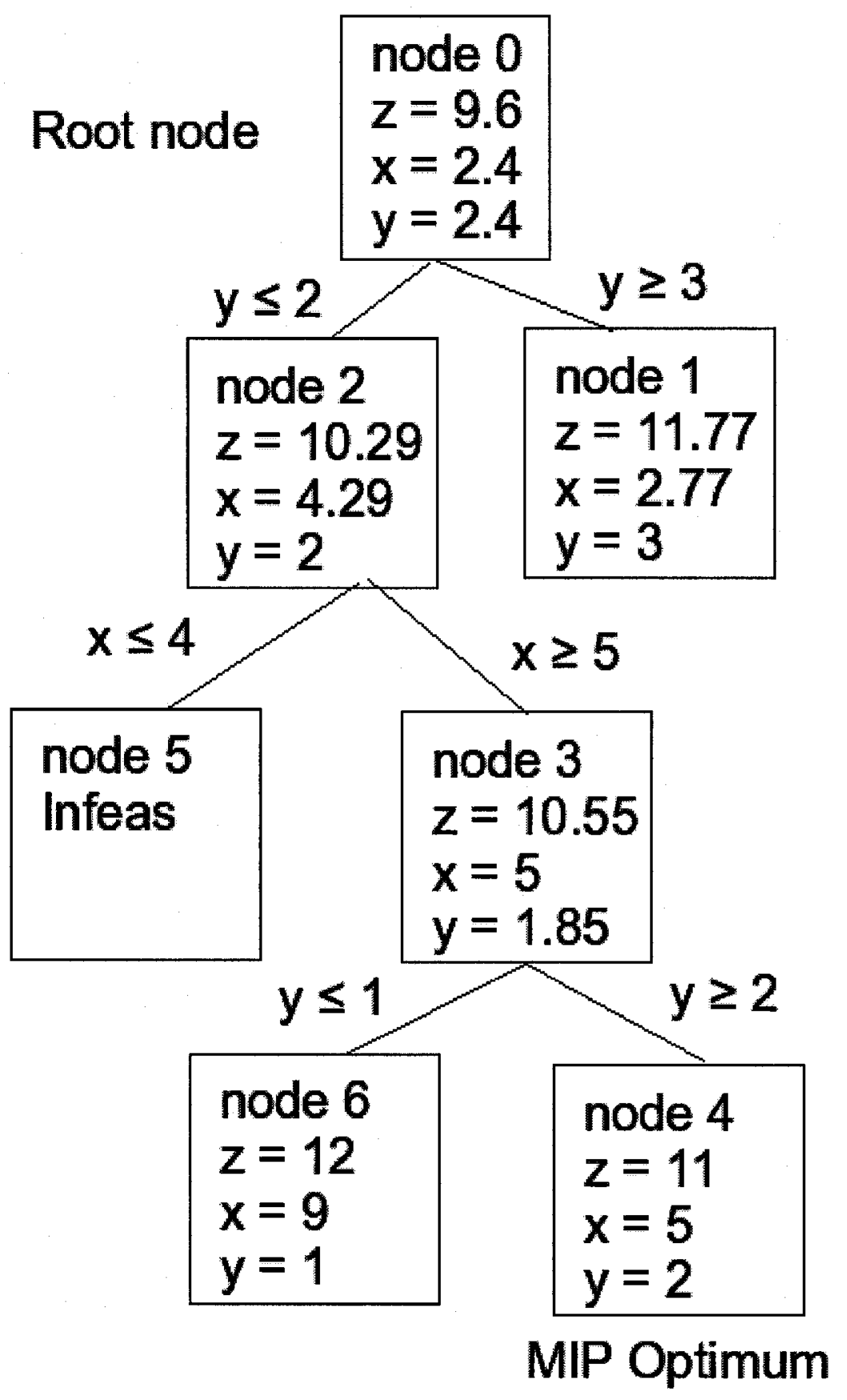

Figure 1.1: Branch and bound tree of MIPEX when it is solved using $D F-B F-A S P=$ 11. 


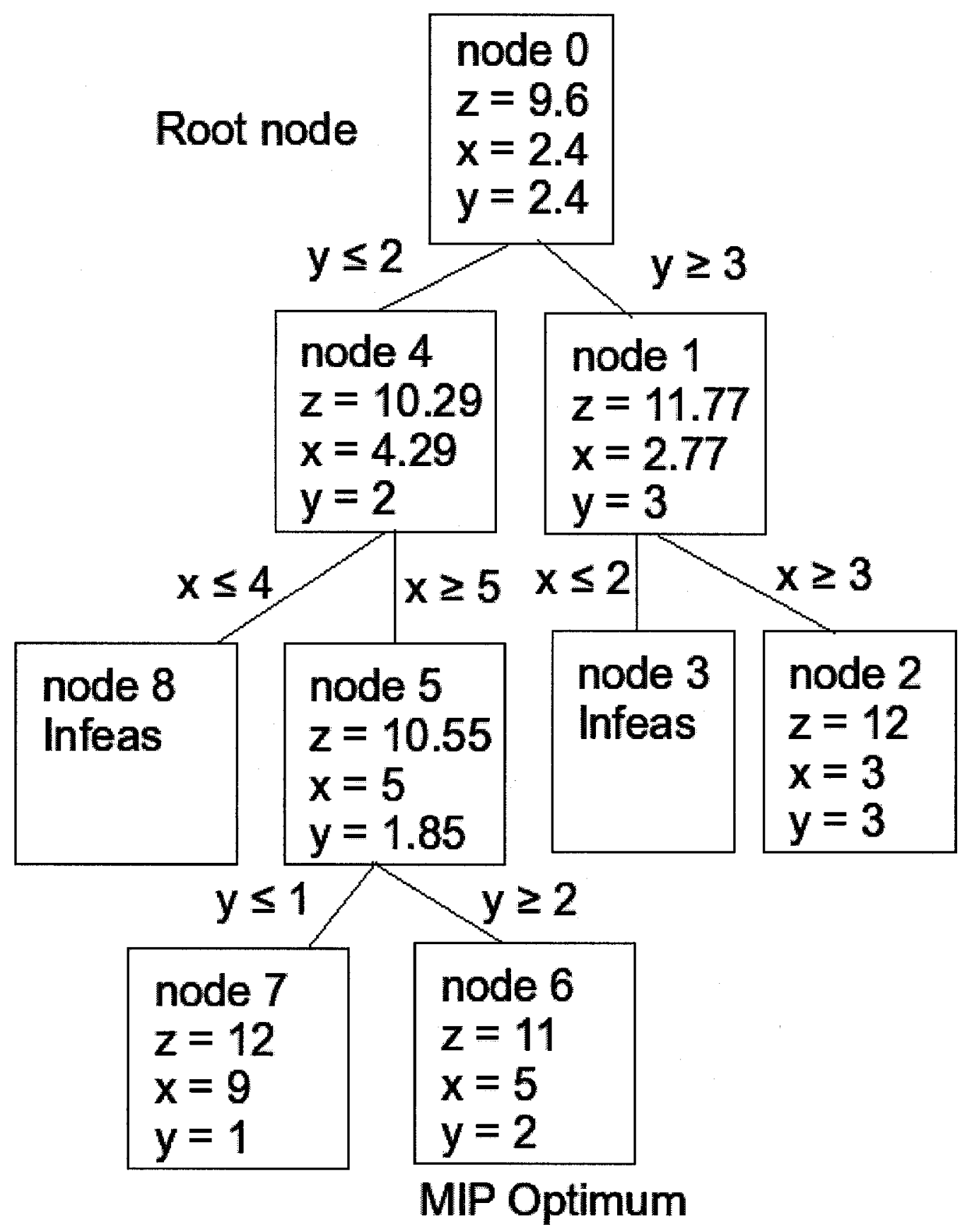

Figure 1.2: Branch and bound tree of MIPEX when it is solved using $D F-D F$. 
In this thesis, three backtracking node selection and two backtrack triggering methods are proposed.

A modification to the best-projection method is proposed in Section 5.1 that estimates the objective value of the best MIP feasible solution attainable from any node using nodes that are not MIP feasible, thus eliminating the requirement of finding an incumbent solution before computing an estimate. This method can be used for backtracking node selection and for backtrack triggering.

A backtracking node selection method is proposed in Section 5.2 that balances the goals of reaching MIP feasibility and MIP optimality by using probability distributions to model these measures based on data from all nodes solved so far. This method dynamically adjusts the weights of the feasibility and optimality goals so that neither can dominate the other for too long, and to favour choosing nodes with lower objective values and lower MIP infeasibility.

The active node search threshold (ANST) proposed in Section 5.4 determines when the cost of using a backtracking node selection method outweighs its benefit. If the cost of a method does outweigh its benefit, then a simpler node selection method is used instead.

A backtrack triggering method is proposed in Section 5.3 that estimates the value of $z^{*}$ based on the objective values of nodes at the estimated depth of a MIP optimal node. The depth of a MIP optimal node is estimated using linear extrapolation of MIP infeasibility along each path of the branch and bound tree.

Empirical results of the experiments are presented in Chapter 7. These results show that the ANST, the modified best-projection method, and the distribution backtracking node selection method proposed in this work greatly speed up branch and bound for solving MIPs. The distribution node selection method outperforms all of the other node selection methods with respect to either finding good MIP feasible solutions or reducing the provable optimality gap for very difficult MIPs. The modified best-projection and the feasibility depth extrapolation backtrack triggering methods speed up the MIP solution process when used with either the modified best-projection 
or the distribution backtracking node selection methods. 


\section{Chapter 2}

\section{A Review of Mixed Integer Programming}

\subsection{Definition of Mixed Integer Program}

Linear Mixed Integer Programs all conform to the following generic formulation. The objective function is defined as

$$
\operatorname{minimize} z=\sum_{j \in I} o_{j} x_{j}+\sum_{i \in C} o_{i} x_{i}
$$

The constraints are defined as

$$
\begin{gathered}
\sum_{j \in I} a_{k j} x_{j}+\sum_{i \in C} a_{k i} x_{i}\{\leq,=, \geq\} v_{k}, \quad k=1, \ldots, k_{\max } \\
l_{j} \leq x_{j} \leq u_{j} \quad \forall j \in I \cup C \\
x_{j} \in \mathbb{Z} \quad \forall j \in I \\
x_{i} \in \mathbb{R} \quad \forall i \in C
\end{gathered}
$$

$z$ : the objective value

$x_{j}$ : a variable 
$o_{j}:$ the objective value coefficient for $x_{j}$

$a_{k j}:$ the constraint coefficient for $x_{j}$ in constraint $k$

$v_{k}$ : the bound on constraint $k$

$l_{j}:$ the lower bound on the value of $x_{j}$

$u_{j}:$ the upper bound on the value of $x_{j}$

$I$ : If $j \in I$ then in order for a solution to be MIP feasible, the value of $x_{j}$ is required to be an integer.

$C:$ If $j \in C$ then the value of $x_{j}$ can be any Real number.

Any maximization problem can be transformed into a minimization problem by multiplying the objective function by -1 .

\subsection{The Branch and Bound Method}

Many methods for solving MIPs employ a branch and bound methodology that involves relaxing the integer constraints to get a lower bound on the solution. This means solving the problem as if it is a purely continuous linear program (LP), commonly called the initial LP-relaxation. If all variables that are constrained to be integer valued in the MIP are integer valued in the solution to the initial LP-relaxation, then this solution is the MIP optimum. Otherwise, the integer constraints are incorporated into the LP formulation by recursively subdividing the feasible region and solving each subdivision until a MIP feasible solution is found. The LP-relaxations are commonly solved using the simplex method since the simplex solution information of any LP-relaxation can be used to speed up the solution of LP-relaxations with small differences in formulation.

The most commonly used method for subdividing the feasible region to solve MIPs proceeds by selecting a candidate variable, $x_{j}$, and then creating two subproblems 
which differ from the initial LP-relaxation only in the bounds on this variable. A candidate variable of an LP-relaxation is a variable that is constrained to be integer valued (referred to as an integer variable) in the MIP but has a fractional value in the solution to the LP-relaxation. In one subproblem the lower bound of $x_{j}$ is set to the nearest integer value that is greater than the LP-relaxation solution value of $x_{j}$. In the other subproblem the upper bound is set to the nearest integer that is less than the value of $x_{j}$. These are referred to as the $u p$ and down branches respectively. This is called branching on variable $x_{j}$. One of the subproblems is then chosen to be solved. If the subproblem solution is LP feasible but not MIP feasible then a candidate variable is chosen and branched on as before.

This process is repeated to create a binary tree where the root node is the initial LP-relaxation and each child node represents a subproblem created by branching on a chosen variable. This method of branching ensures that the feasible region of a node excludes the solution to the parent node and therefore the value of $z$ at a node, commonly referred to as the lower bound of a node, will be greater than or equal to the lower bound of its parent. At an intermediate point in the MIP solution, the MIP feasible solution with the currently lowest value of $z$ is commonly called the incumbent solution and its value of $z$ is the upper bound of the MIP objective value. This means that a node need not be explored if its lower bound is greater than that of the incumbent solution. An incumbent solution is optimal when all nodes with a lower bound less than that of the incumbent have been explored. The branch and bound solution method is summarized in Algorithm 1. 
Algorithm 1 (Branch and Bound)

Input:

MIP instance. Incumbent solution, $X_{\text {inc }}=\phi$.

Objective value of incumbent solution, $z\left(X_{i n c}\right)=\infty$. List of unexplored nodes, $L_{u}=\phi$.

Procedure:

1. Add the initial LP-relaxation to $L_{u}$ as the root node. current Node $=$ root node.

2. Solve the LP-relaxation of the root node.

- If the LP-relaxation is infeasible then exit with MIP infeasible outcome.

- If the LP-relaxation is integer-feasible then exit with relaxation solution as optimum.

3. Choose a candidate variable in current Node for branching.

4. Create two child nodes from currentNode by branching on the selected variable and add these new nodes to $L_{u}$. Remove current Node from $L_{u}$.

5. Choose a node from $L_{u}$ for exploration and label it currentNode.

6. Solve the LP-relaxation for currentNode.

- If LP-relaxation is infeasible or it is feasible with a lower bound that is greater than $z\left(X_{i n c}\right)$ then discard currentNode and go to Step 8.

- If LP-relaxation is MIP feasible then:

- If LP-relaxation objective function value is less than $z\left(X_{i n c}\right)$ then replace $X_{\text {inc }}$ with this solution, else discard currentNode.

- Go to Step 8

7. Go to Step 3

8. If list of unexplored nodes is empty then:

7.1 If $X_{i n c}=\phi$, then exit with infeasible outcome.

7.2 Optimum is $X_{i n c}$ : exit with optimal outcome.

9. Go to Step 5 


\subsubsection{Branching Variable Selection}

If the solution to the initial LP-relaxation has more than one candidate variable then selecting which one to branch on may have a significant impact on the performance of the branch and bound method. Therefore, it is important to make a good choice. A prevalent paradigm in branching variable selection methods is to pick the variable which will lead to the largest change in the value of $z$ between the current node and one of its children [26]. Towards this end, various methods have been developed to estimate the impact that branching on a given variable has on the value of $z$.

Two simple methods for branching variable selection are to

- choose the candidate variable that corresponds to the largest objective coeffcient value, or

- choose the candidate variable that is furthest from having an integer value.

A very computationally expensive way to measure the impact of branching variable choice on the value of $z$ is to try branching on each candidate variable and choosing the subproblem with the largest value of $z$. To reduce the computational cost of this strategy only a promising subset of candidate variables are branched on and the solution process of the subproblems is interrupted after a given number of simplex iterations [26, 6]. This method is referred to as strong branching.

An observation made by Bènichou et al. [7] is that branching on a given variable had a similar effect on the change in the value of $z$ at different nodes. This observation led to what is called pseudo-cost branching in which the effect that branching on a variable at a given node has on the value of $z$ is used to estimate this effect at a different node.

Driebeek [14] proposed a method, which was later improved upon by Tomlin [38], in which the minimum change in objective value between a parent and child node when branching on a given candidate variable is computed using its dual cost and a constraint coefficient in the simplex tableau for the candidate variable. 
A class of methods developed by Patel and Chinneck [33] focus on choosing the branching variable that has the largest impact on the active constraints at the LPrelaxation solution for the current node. The goal of these methods is to decrease the amount of time required to find a MIP feasible solution. These methods involve analyzing the values of $a_{i j}$ corresponding to candidate variables and the active constraints. Active constraints are those at which the current solution satisfies

$$
\sum_{j \in I} a_{k j} x_{j}+\sum_{i \in C} a_{k i} x_{i}=v_{k}
$$

\subsubsection{Node Selection}

If there are two or more unexplored (active) nodes then the method of choosing which node to explore next can have a significant impact on the performance of the branch and bound method. When selecting nodes to explore, the goal is to choose nodes that are ancestors of a MIP optimal node. No method has been discovered, yet, that accurately determines whether or not an unexplored node is an ancestor of a MIP optimal node. Many node selection heuristic methods have been proposed to achieve this goal, or that use some other strategy of developing the branch and bound tree to achieve a different goal.

When choosing a node to explore, it is most efficient to avoid choosing one whose lower bound is greater than the (unknown) MIP optimal objective value, $z^{*}$. Such a node is commonly called a superfluous node. Since the LP-relaxation of an unexplored node has not been solved yet, the lower bound of each unexplored node is initially set equal to that of its parent node. This is also the case for any other LP solution characteristics of a node such as the number of candidate variables.

Many implementations of the branch and bound method use an estimate of the value of $z^{*}$ to avoid the exploration of superfluous nodes. An estimate of $z^{*}$ that is used for the purpose of avoiding the exploration of superfluous nodes is referred to as an aspiration value. The aspiration value is typically set by the user before the branch and bound process begins. 
The state of the art in heuristic node selection methods is covered in depth in Chapter 3. 


\section{Chapter 3}

\section{State of the Art in Heuristic Node Selection Methods}

\subsection{Basic Node Selection Methods}

The best-first, or best-bound, node selection method avoids the exploration of superfluous nodes by choosing the unexplored node whose lower bound is the lowest of all the unexplored nodes. The best-first method causes the tree to be searched in such a way that nodes that are closer in the tree to the root node are more likely to be chosen (because the lower bounds are generally worse deeper in the tree). Since MIP feasible solutions usually occur at nodes that are far from the root node, the best-first search method is not a good choice for quickly finding a MIP feasible solution. This is also true of the breadth-first node selection method, which chooses the earliest created active node.

If the goal is to find a MIP feasible solution as quickly as possible then it is probably better to use depth-first node selection. After a node is solved and a branching variable is chosen, depth-first chooses one of that node's children to solve next. If both children are unsolved, then some other criteria is used to determine which child to explore first. Some common methods for choosing a branch direction are to choose the up branch, the down branch, the branch of the nearest integer value for the 
branching variable, or the branch that forces the value of the branching variable away from this variable's value at the root node [28]. If the last solved node is either LP infeasible, MIP feasible, or worse than the incumbent solution, then the last created active node is explored.

In depth-first node selection the most recently created unexplored node is explored first, so the difference in formulation between successive nodes solved is usually very small. Best-first node selection, as well as most other methods, cause the tree to be searched in such a way that the formulations of successive nodes solved are likely to be much different. A consequence of this is that the average number of simplex iterations to solve each node will be significantly higher using best-first versus depthfirst node selection $[6,12,26]$. This assumes that the simplex basis of only the most recently solved node is available which, due to memory limitations of the computers on which they are executed, is true for most MIP solvers. Another consequence of using the best-first search is that the number of unexplored nodes will likely increase much more quickly than if depth-first search is used and, therefore, is more likely to become too large for the amount of available memory. Some MIP solvers, such as SCIP [4], use a predefined threshold of memory usage to ensure that the size of the branch and bound tree does not exceed the available memory by switching to depth-first node selection if this threshold is exceeded.

Another node selection method that is used to find incumbent solutions quickly is the most-feasible method. This method chooses the node with the smallest sum of integer infeasibilities, $s_{i}$. The value of $s_{i}$ is computed using Equation 3.2 where

$$
\begin{gathered}
f_{j}=x_{j}-\left\lfloor x_{j}\right\rfloor \\
s_{i}=\sum_{j \in I} \min \left\{f_{j}, 1-f_{j}\right\}
\end{gathered}
$$

Many node selection schemes have been proposed to combine the benefits of bestfirst and depth-first methods. One such scheme uses depth-first search until a MIP 
feasible solution is found and then switches back and forth between best-first and depth-first strategies [6].

Backtracking node selection uses depth-first search until the current node is either: MIP feasible, LP infeasible, has a lower bound that is greater than the value of $z$ of the incumbent solution, or is considered undesirable to explore, at which point backtracking occurs. When backtracking occurs, a backtracking node selection method is used to select the next node to explore. Typically either the best-first, best-projection, or best-estimate criterion is used to select the next node to be explored [26].

\subsection{Estimate based Node Selection}

Knowing the value of $z$ of the best possible MIP feasible solution attainable at a descendent of a given node would be useful in selecting which node to explore. Methods which estimate this value, called estimate based methods, include the best-projection method $[19,29]$ and the best-estimate method $[7,15]$. In these methods the active node with the smallest such estimate is explored next.

\subsubsection{Best-Projection Node Selection}

The best-projection method $[19,29]$ uses the change in the value of $z$ between the root node and the incumbent solution as well as the change in the sum of integer infeasibilities between the root node and the node for which the estimate is being computed. The best projection estimate at node $i$ is computed using Equation 3.3 where $z_{i}$ is the lower bound of node $i, z_{i n c}$ is the value of $z$ for the incumbent solution, $z_{0}$ is the lower bound of the root node, $s_{0}$ (see Equation 3.2) is the sum of integer infeasibilities at the root node, and $s_{i}$ is the sum of integer infeasibilities at node $i$.

$$
E_{i}=z_{i}+\left(\frac{z_{i n c}-z_{0}}{s_{0}}\right) s_{i}
$$

The rationale for this method is that the change in the value of $s_{i}$ between a 
parent and child node is proportional to the change in the value of $z$ between these nodes. This influence is approximated using the incumbent solution and is used to determine how much the value of $z_{i}$ will have to change in order for the value of $s_{i}$ to be reduced to 0 if this node is explored. If no incumbent solution has been found, then a backtracking node selection method that emphasizes finding MIP feasible solutions quickly, such as the depth-first method or the most-feasible method, is first used until an initial incumbent is found.

\subsubsection{Best-Estimate Node Selection}

The best-estimate method $[7,15]$ uses pseudo-cost estimates. Each integer variable has two pseudo-cost values associated with it, one for the up branch, $P_{j}^{U}$, and the other for the down branch, $P_{j}^{D}$. When a variable is branched on, the change in the lower bound between the parent node and the down child node is $\Delta z_{j}^{D}=z_{j}^{D}-z_{j}^{P}$ where $z_{j}^{D}$ is the lower bound for the down child node and $z_{j}^{P}$ is the lower bound for the parent node. Likewise for the up child node $\Delta z_{j}^{U}=z_{j}^{U}-z_{j}^{P}$. The pseudo-cost value for the down branch of $x_{j}$ is

$$
P_{j}^{D}=\Delta z_{j}^{D} / f_{j}
$$

and similarly for the up branch

$$
P_{j}^{U}=\Delta z_{j}^{U} /\left(1-f_{j}\right)
$$

The value of $f_{j}$ is defined in Equation 3.1.

Updating the pseudo-costs for $x_{j}$ is typically done by averaging the values from every instance that $x_{j}$ was branched on. The estimate of the best integer feasible solution attainable from a node $i$ is calculated using Equation 3.4.

$$
E_{i}=z_{i}+\sum_{j \in I} \min \left\{P_{j}^{D} f_{j}, P_{j}^{U}\left(1-f_{j}\right)\right\}
$$


If there is no up pseudo-cost estimate for a candidate variable then the up pseudocost estimate for this variable is set to the average of the up pseudo-costs of all of the variables with at least one up pseudo-cost estimate. If there are no pseudo-cost estimates for all other variables then set the up pseudo-cost to 1 . This is also done for the down pseudo-cost [3].

Forrest et al. [15] proposed a method that uses best-estimate node selection until a MIP feasible solution is found and then chooses nodes according to the percentage error criterion computed for a node $i$ using Equation 3.5.

$$
P E_{i}=100 \times \frac{E_{i}-z_{i n c}}{z_{i n c}-z_{i}}
$$

The percentage error method chooses the node with the smallest value of $P E_{i}$. The idea behind this method is to determine how much error there is in the estimate $E_{i}$ in order for the current incumbent solution to not be optimal. If, for all nodes, $E_{i}<z_{i n c}$, then $P E_{i}<0$ and nodes with larger $z_{i}$ are favored. If $E_{i} \geq z_{i n c}$ for all nodes, then nodes with smaller $z_{i}$ are favored.

Achterberg [3] proposed interleaving best-estimate with best-first node selection by performing best-first node selection once for every $b_{f q}$ best-estimate backtracking node selections. It is suggested that $b_{f q}=9$ should be used.

Linderoth and Savelsbergh [26] considered the pseudo-cost estimates to be optimistic in that they assume that rounding the value of each candidate variable such that the value of $E_{i}$ is minimized will result in feasible solutions. They propose an alternative to Equation 3.4 in which there is a probability, $q_{j}$, associated to each candidate variable indicating the likelihood that the variable will be rounded to the nearest integer in an integer feasible solution. The adjusted pseudo-cost is computed using Equation 3.6. 


$$
\begin{aligned}
E_{i}= & z_{i}+\sum_{j \in I: f_{j} \leq 0.5} f_{j} P_{j}^{D} q_{j}+\left(1-f_{j}\right) P_{j}^{U}\left(1-q_{j}\right) \\
& +\sum_{j \in I: f_{j}>0.5} f_{j} P_{j}^{D}\left(1-q_{j}\right)+\left(1-f_{j}\right) P_{j}^{U} q_{j}
\end{aligned}
$$

The value of each $q_{j}$ is computed by performing an LP-based successive rounding heuristic algorithm starting at an LP-relaxation solution $\mathbf{x}$ and recording how often the value of each integer infeasible variable was rounded to an integer value whose difference is greater than 0.5 from its initial value, to create a MIP feasible solution $\hat{\mathbf{x}}$. This flip percentage, $\zeta$, is

$$
\zeta=\frac{\left|\left\{j \in I:\left|\hat{x}_{j}-x_{j}\right|>0.5\right\}\right|}{|I|} .
$$

The value of each $q_{j}$ is then calculated as

$$
q_{j}=1-\zeta
$$

The value of $q_{j}$ is modified to reflect the likelihood that a variable that is very close to integer valued will be rounded to the nearest integer value in a MIP feasible solution. Another modification to $q_{j}$ is based on the frequency at which $x_{j}$ takes on a given value over all of the MIP feasible solutions.

\subsection{Genetic Programming and Node Selection}

Kostikas and Fragakis [21, 22] have experimented with using genetic programming (GP) to create customized node selection rules for a given MIP instance during the branch and bound solution process of this instance. The method proceeds by running branch and bound using a standard node selection method until a set of criteria are met. GP is then run using some characteristics of the current branch and bound tree 
as a training set, and a predefined function set, to evolve a node selection rule that is then used for the remainder of the branch and bound process. Node characteristics such as depth in the tree and the number of candidate variables are examples of the branch and bound tree characteristics used in the training set. The function set contains several arithmetic and logical operations such as "add", "subtract" and "or".

\subsection{Triggering Backtracking}

If an estimate $\tilde{z}^{*}$ of the MIP optimal objective value for a problem is computed early during the branch and bound process then backtracking node selection can be initiated when the lower bound of a node exceeds this estimate. A value that is used to trigger backtracking in this way is called an aspiration value. The purpose of using an aspiration value is to avoid exploring superfluous nodes while exploring the tree more efficiently than when using best-first node selection. The aspiration value is typically set by the user before the beginning of the branch and bound process.

It should be noted that active nodes with $z>\tilde{z}^{*}$ are not removed from the tree because it is not guaranteed that $\tilde{z}^{*} \geq z^{*}$ using heuristic methods of estimating $z^{*}$, so all of these active nodes are eligible to be explored. If $\tilde{z}^{*}>z^{*}$ and $\tilde{z}^{*}$ is not too large, then hopeless dives will be stopped and the amount of effort required to solve a MIP will likely be smaller than if no aspiration value is used. If $\tilde{z}^{*}$ is too large, then backtracking is triggered only when a leaf node is solved so the amount of effort required to solve a MIP is greater than not using an aspiration value due to the cost of estimating $z^{*}$. If $\tilde{z}^{*}$ is too small, then backtracking is triggered after each node is solved. For some MIP instances and backtracking node selection methods, triggering backtracking after every node is solved increases the amount of effort required to solve these instances relative to not using an aspiration method, whereas the opposite is true for others.

Various methods have been proposed to compute $\tilde{z}^{*}$ including best-projection estimates [26] as well as some estimation methods based on pseudo-costs $[17,26]$. 
The idea of using an estimate of the objective value of the best integer feasible solution attainable from a given node as an aspiration value was first proposed by Gauthier and Ribière [17]. They used a pseudo-cost based estimate similar to the one in the best-estimate node selection method described in Section 3.2.2. Linderoth and Savelsbergh [26] revisited the idea by experimenting with using the best-projection and pseudo-cost estimates as well as proposing a modification to the pseudo-cost method.

If $E_{i}$ is the estimate of the best integer feasible solution attainable from node $i$, then the estimate $\tilde{z}^{*}$ of $z^{*}$ is taken as the smallest $E_{i}$ over all of the active nodes in the tree as shown in Equation 3.7.

$$
\tilde{z}^{*}=\min _{i \in L_{u}}\left\{E_{i}\right\}
$$

Gauthier and Ribière [17] proposed calculating an upper bound, $Q$, for $z$ using $\tilde{z}^{*}$ as follows:

$$
Q=\tilde{z}^{*}+\rho\left(\tilde{z}^{*}-z_{0}\right)
$$

where $\rho$ is a parameter which is usually set to 0.5 .

Many backtrack triggering methods that do not involve computing an estimate of $z^{*}$ have also been implemented in many of the currently available MIP solvers.

A backtrack triggering method used in CPLEX [6] has a parameter whose value affects how frequently backtracking occurs [6]. Backtracking occurs when the difference between the objective value of the most recently solved node and the current lower bound on the MIP optimal objective value $z_{l b}$ exceeds the difference between the incumbent objective value and $z_{l b}$ multiplied by this parameter.

Xpress-MP [6] has a method that triggers backtracking after every solved node until a user-defined number of backtracks have occurred. Once enough backtracks have been performed, the backtracking trigger is changed so that backtracking occurs when the most recently solved node is a leaf.

The idea of switching between triggering backtracking after every node is solved 
and triggering backtracking after a leaf node is solved is also used in ABACUS [1] and $B c p$ [35]. These methods are described in Section 3.5.

The SCIP [4] MIP solver triggers backtracking if at least one of the following conditions is satisfied:

- the number of nodes solved during the current dive exceeds a minimum value that is set to 0.1 times the maximum depth of all solved nodes, and $\gamma_{i}>\gamma_{\max }$ where $\gamma_{i}$ is computed using Equation 3.8.

$$
\gamma_{i}=\frac{z_{i}-z_{l b}}{z_{i n c}-z_{l b}}
$$

- the number of nodes solved during the current dive exceeds a maximum value that is set to 0.5 times the maximum depth of all solved nodes.

Symphony [34] has three options available to the user for determining when to backtrack from a dive. The first option is to not backtrack until the last solved node has no child nodes. The second option is to backtrack if the difference between the average of the objective values of the best $k$ solved nodes and the objective value of the most recently solved node is greater than a threshold that is computed using a user-defined parameter. The last option is to backtrack if the difference between the average of the lower bounds of the best $k$ solved nodes and the objective value of the most recently solved node is greater than a given fraction of the gap between this average and the upper bound of the MIP optimal objective value.

\subsection{Solver Specific Heuristic Node Selection Meth- ods}

In addition to the standard methods, some solvers have their own customized heuristic backtracking node selection and backtrack triggering methods. Some solvers also have seldom used heuristic node selection methods not included in the previous sections. 
In some solvers such as CPLEX and Xpress-MP, the details of some of these methods are not given in the literature.

$C P L E X$ [6] has a custom backtracking node selection method based on pseudocosts as well as a method that probes both children of the most recently solved node.

Xpress-MP [6] uses best-first backtracking node selection for a given number of times, and then the backtracking node selection method used is changed to a method chosen by the user.

$S C I P$ [4] has two custom backtracking node selection methods. One method orders nodes according to a weighted sum of the best-estimate value of $E_{i}$ (Equation 3.4 ) and the lower bound of a node. The other uses depth-first node selection and periodically selects a node according to the best-first method. A user-defined parameter determines how often best-first search is used.

$C b c$ [16] has a node selection method that switches between depth-first, least number of candidate variables first, best-first, and a weighted sum of the objective value with the number of candidate variables. The choice of method depends on a user-defined parameter value as well as the number of MIP feasible solutions found, the smallest lower bound over all of the active nodes, and the number of nodes in the tree.

MINTO [25] has a hybrid method that combines the depth-first and best-estimate methods.

$L p \_s o l v e$ [25] has a two-phase method that switches between depth-first and breadthfirst node selection.

Symphony [34] uses depth-first dives at random intervals that are controlled by a user-defined parameter that determines the approximate frequency of these dives.

Lindo [6] has a node selection option that chooses the node with the worst objective value, worst-first. Another option alternates between the depth-first, best-first, worst-first, and best-estimate node selection methods.

$A B A C U S$ [1] has a two-phase node selection method that uses depth-first backtracking until a MIP feasible solution is found, then it uses best-first node selection 
with backtracking triggered after every node is solved.

$B c p$ [35] uses best-first backtracking node selection with backtracking triggered after every node is solved except if the number of candidate variables of the most recently solved node is small or this node is determined to be "close" to the best node according to a user-defined parameter value. If this exception occurs, then backtracking is triggered when the most recently solved node is a leaf.

BonsaiG [18] has an option for ordering node selection according to the weighted sum of the best-projection estimate with the sum of infeasibilities defined by Equation 3.2. Backtracking is triggered after every node is solved.

\subsection{State of the Art in MIP Software}

This section summarizes the available software for solving MIPs, and the node selection methods that are provided in each solver. The available MIP solvers are those examined in $[25,30,6]$. These solvers are shown in Table 3.1 along with the corresponding options available for the node selection methods described below.

DF: Depth-first (Section 3.1).

BR: Breadth-first (Section 3.1).

BP: Best-projection (Section 3.2.1).

BF: Best-first (Section 3.1).

BE: Best-estimate (Section 3.2.2).

BT: Custom heuristic backtrack triggering methods (Sections 3.4 and 3.5)

UA: User defined fixed aspiration value (set to infinity by default)

OC: Other custom heuristic node selection methods (Section 3.5) 
Table 3.1: MIP solver software and available standard backtracking node selection methods.

\begin{tabular}{|l|c|c|c|c|c|c|c|c|}
\hline Solver & DF & BR & BP & BF & BE & BT & UA & OC \\
\hline \hline CPLEX 11.0 [6] & $\mathrm{x}$ & & & $\mathrm{x}$ & $\mathrm{x}$ & $\mathrm{x}$ & $\mathrm{x}$ & $\mathrm{x}$ \\
\hline Xpress-MP [6] & $\mathrm{x}$ & & & $\mathrm{x}$ & $\mathrm{x}$ & $\mathrm{x}$ & $\mathrm{x}$ & \\
\hline SCIP [4] & $\mathrm{x}$ & & & $\mathrm{x}$ & $\mathrm{x}$ & $\mathrm{x}$ & & $\mathrm{x}$ \\
\hline Cbc [16] & $\mathrm{x}$ & & & $\mathrm{x}$ & $\mathrm{x}$ & & & $\mathrm{x}$ \\
\hline MINTO [37] & $\mathrm{x}$ & & $\mathrm{x}$ & $\mathrm{x}$ & $\mathrm{x}$ & & & $\mathrm{x}$ \\
\hline GLPK [27] & $\mathrm{x}$ & $\mathrm{x}$ & $\mathrm{x}$ & $\mathrm{x}$ & & & & \\
\hline lp_solve [20] & $\mathrm{x}$ & & & $\mathrm{x}$ & & & $\mathrm{x}$ & $\mathrm{x}$ \\
\hline Symphony [34] & $\mathrm{x}$ & $\mathrm{x}$ & & $\mathrm{x}$ & & $\mathrm{x}$ & $\mathrm{x}$ & $\mathrm{x}$ \\
\hline Lindo [6] & $\mathrm{x}$ & & & $\mathrm{x}$ & $\mathrm{x}$ & & & $\mathrm{x}$ \\
\hline ABACUS [1] & $\mathrm{x}$ & $\mathrm{x}$ & & $\mathrm{x}$ & & & & $\mathrm{x}$ \\
\hline Bcp [35] & $\mathrm{x}$ & & & $\mathrm{x}$ & & $\mathrm{x}$ & & \\
\hline BonsaiG [18] & & & $\mathrm{x}$ & & & & & $\mathrm{x}$ \\
\hline
\end{tabular}




\section{Chapter 4}

\section{The Node Selection Problem}

The goal of this thesis is to develop new node selection methods that reduce the amount of effort required to solve mixed-integer programs. This is a desirable outcome because some MIP instances require an impractical amount of time to solve. Towards this end, this work develops methods to order the nodes during the node selection process in branch and bound so as to quickly find good incumbent solutions and to avoid the exploration of superfluous nodes. Backtracking node selection is used as the framework for the development of node selection methods in this thesis. Specifically, the focus of this work is on developing node selection methods for use during backtracking, and methods of determining when to backtrack.

\subsection{Performance of the State of the Art}

\subsubsection{Backtracking Node Selection}

There are no documented empirical studies that show that any one of the state of the art backtracking node selection methods described in Chapter 3 greatly outperforms all of the other methods. The empirical study presented in [26] shows that, over all of the tested MIP instances, the average ranking of the best node selection method is 5.42 out of the 13 tested node selection methods. The empirical study presented in [22] 
shows that, over all of the tested MIP instances, the average ranking of the best node selection method is 2.46 out of the 6 tested node selection methods. The empirical study presented in [3] shows that the best 4 out of the 7 tested node selection methods are within $4 \%$ of each other with respect to a time based performance measure.

The survey of the state of the art in node selection presented in Chapter 3 shows that none of the currently available node selection methods exploit correlation or distribution characteristics of the branch and bound tree. There is also no documented consideration given to the cost versus the benefit of using any of the state of the art backtracking node selection methods. Therefore, there is an opportunity to exploit these ideas to develop better backtracking node selection methods.

\subsubsection{Backtrack Triggering}

The survey of the state of the art MIP software presented in Section 3.6 shows that no MIP software uses a method for estimating the MIP optimal objective value for triggering backtracking. This survey also shows that all of the backtrack triggering methods available in the state of the art MIP software rely on user set parameter values. Another deficiency is that there is very little documentation giving details of how these methods work and there are no documented empirical results that demonstrate that these methods work well. Some MIP software allows the user to set the aspiration value, which indicates that such a value may be useful in reducing the amount of effort required to solve a MIP; however, an empirical study presented in [26] shows that the state of art methods for estimating the optimal objective value do not significantly reduce the amount of effort required to solve most MIPs.

A proof-of-concept experiment was performed to demonstrate the potential value of using a good aspiration value on the performance of branch and bound. The experiment demonstrates how using a priori knowledge of the MIP optimal objective value to trigger backtracking results in a reduction of the effort required to solve MIPs relative to the state of the art backtrack triggering methods. In this experiment each MIP instance is solved using NONA backtrack triggering (see Page 78 for the 
definition of NONA) to determine its optimal objective value. Each MIP instance is then solved using its optimal objective value to trigger backtracking (PERA). Finally, each MIP instance is solved 2 more times, each time using the following state of the art backtrack triggering methods: DBPA and PCAS (see Page 78 for the definitions of DBPA and PCAS).

The details of the experimental setup are given in Chapter 6. The branch and bound options used in this experiment are given below.

- Branching variable selection is performed using the method by Driebeek [14] and Tomlin [38]. This is the best method according to the experiment described in Section 6.5.1.

- Backtracking node selection is performed using the best-projection method (the default method in GLPK) described in Section 3.2.1.

- Gomory's mixed integer cuts are used at the root node. This is the best option according to the experiment described in Section 6.5.2.

In this experiment the solution process for each MIP instance is halted after 1 hour. The 50 MIP instances used for this experiment are listed in Table A.2, excluding instances that are (i) not solved within 1 hour using NONA, or (ii) MIP feasible at the root node.

Table 4.1 shows that using an aspiration value that is equal to the MIP optimal objective value reduced TOTTM (see Page 68 for the definition of TOTTM) by $15 \%$ relative to the second best aspiration method. It also reduced the value of $M R A T E$ (see Page 69 for the definition of $M R A T E$ ) by $48 \%$ relative to the second best aspiration method. This result demonstrates that it is worthwhile developing better ways of estimating the MIP optimal objective value for use in triggering backtracking. The performance profile in Figure 4.1 shows that using a perfect aspiration value solves most of the MIP instances more quickly than the state of the art aspiration methods. 
Table 4.1: Performance of perfect aspiration value versus the state of the art methods. The best values in each of the TOTTM and MRATE columns are in boldface. (see Page 68 for the definition of $F A I L$ )

\begin{tabular}{|l|r|r|r|}
\hline Contender & FAIL & TOTTM & MRATE \\
\hline NONA & 0 & 5670.57 & 1.52 \\
PERA & 0 & $\mathbf{4 7 5 8 . 8 4}$ & $\mathbf{1 . 0 1}$ \\
DBPA & 0 & 5487.83 & 1.5 \\
PCAS & 0 & 5727.81 & 1.59 \\
\hline
\end{tabular}

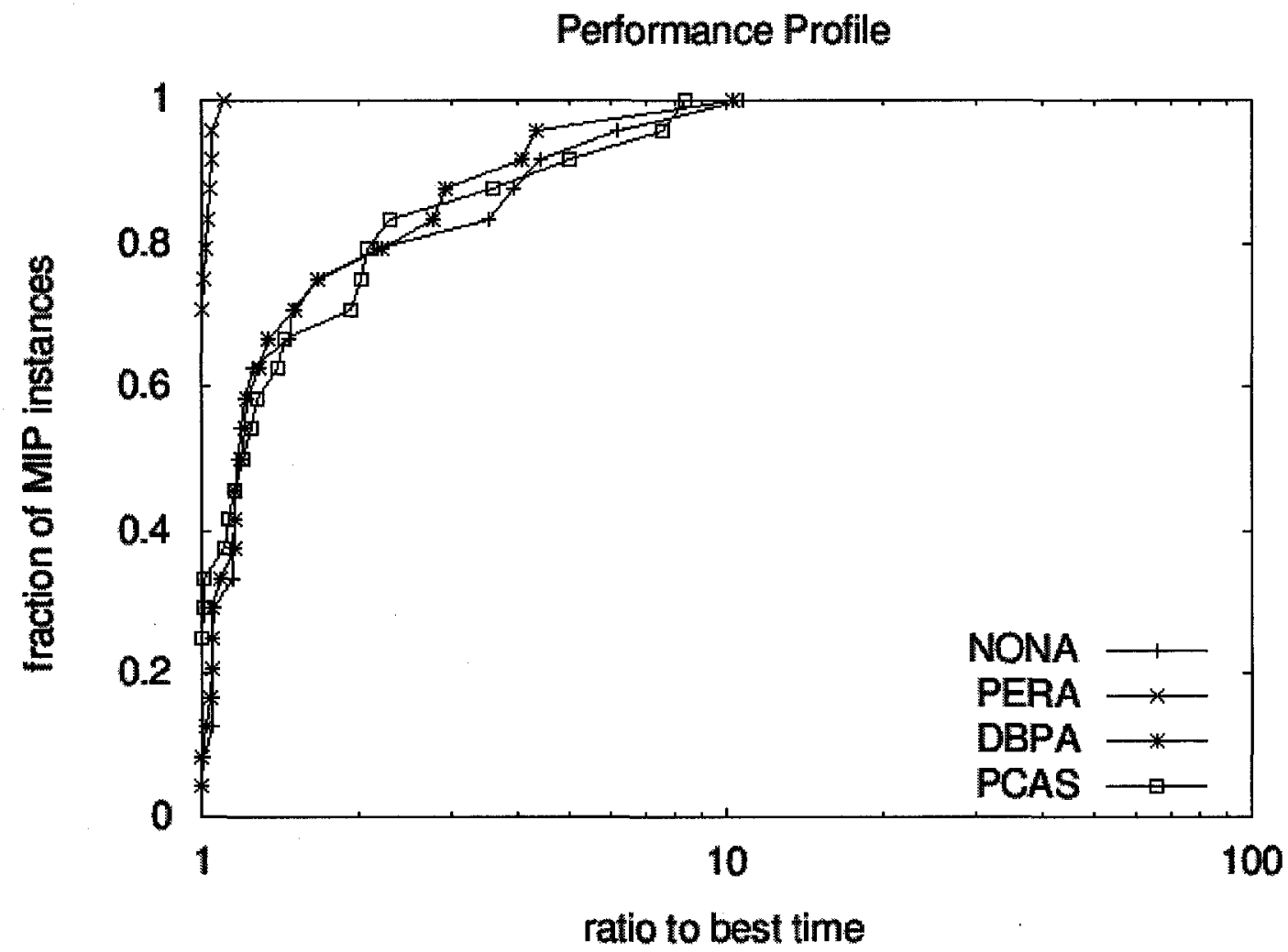

Figure 4.1: A performance profile comparing the NONA, PERA, DBPA, and PCAS options for aspiration method. 


\section{Chapter 5}

\section{New Methods for Backtrack \\ Triggering and Node Selection}

The main contributions of this work on improving the branch and bound method of solving MIPs are:

- the modified best-projection backtracking node selection and aspiration methods;

- the distribution backtracking node selection method;

- the feasibility depth extrapolation aspiration method;

- and the active node search threshold.

This chapter presents the ideas and observations that underlie these methods, and their algorithmic details. 


\subsection{Modified Best Projection Node Selection and Aspiration Method}

A modification to the best-projection method is proposed that can estimate the objective value of the best MIP feasible solution attainable from any node without using an incumbent solution. It is important to be able to compute an estimate without requiring an incumbent solution because an incumbent solution can be very difficult to find in some MIP instances. The modified best-projection method is used as a backtracking node selection method as well as a backtrack triggering method.

This method is based on Observation 1.

Observation 1 (Node Infeasibility versus Optimality) The approximately linear correlation between $z^{\text {min }}(c)$ and $c$ for many MIPs indicates that a reasonably accurate estimate of the MIP optimal objective value may be computed early in the branch and bound process. Note that when a MIP solution is completed successfully, $z^{\text {min }}(0)$ is the MIP optimal objective value.

$c_{i}$ is the number of candidate variables at node $i . c_{0}$ is the number of candidate variables at the root node.

$z_{i}$ is the objective value at node $i . z_{0}$ is the objective value at the root node.

$z^{\min }(c)$ is the smallest $z_{i}$ over all nodes with $c$ candidate variables:

$$
z^{\min }(c)=\min _{i}\left\{z_{i}: c_{i}=c\right\}
$$

For many MIP instances, plots of $z^{\text {min }}(c)$ versus $c$ show a trend that indicates the possibility of estimating the value of $z^{*}$ early in the branch and bound process by simple linear extrapolation. For the dcmulti MIP instance, the trend can be seen in the plot of $z^{\text {min }}(c)$ shown in Figure 5.1 for $10 \leq c \leq 30$. For the fixnet/4 MIP instance, the trend is shown in Figure 5.2 for $5 \leq c \leq 15$. 


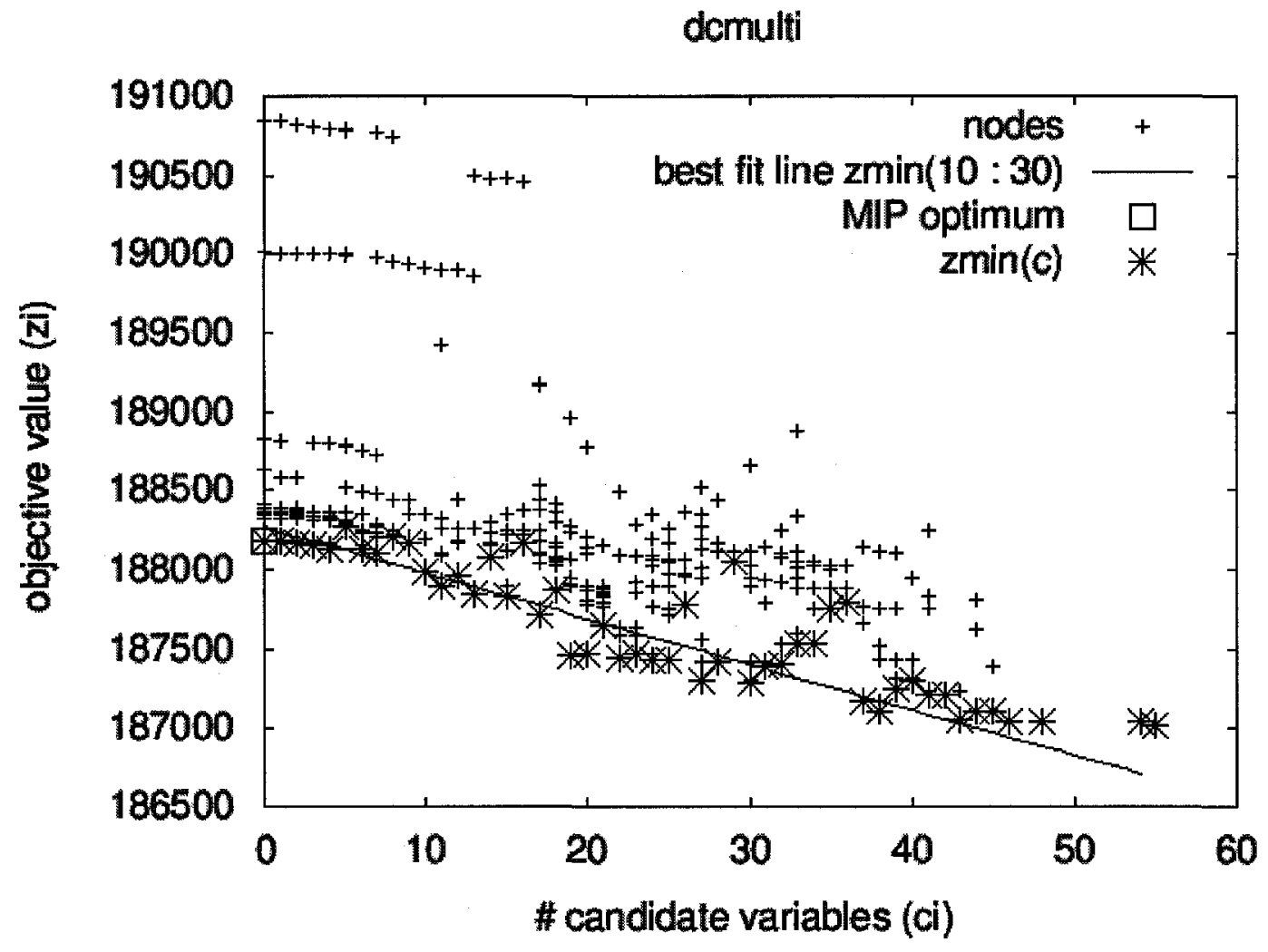

Figure 5.1: Plot of $z_{i}$ and $z^{m i n}(c)$ versus $c_{i}$ for all of the nodes in the tree at the completion of the branch and bound process for the dcmulti MIP instance. Also shown is the best fit line through $z^{\min }(c)$ for $10 \leq c \leq 30$. The DEBP-NONANOAN node selection configuration is used (see Chapter 7 for the configuration definitions). 


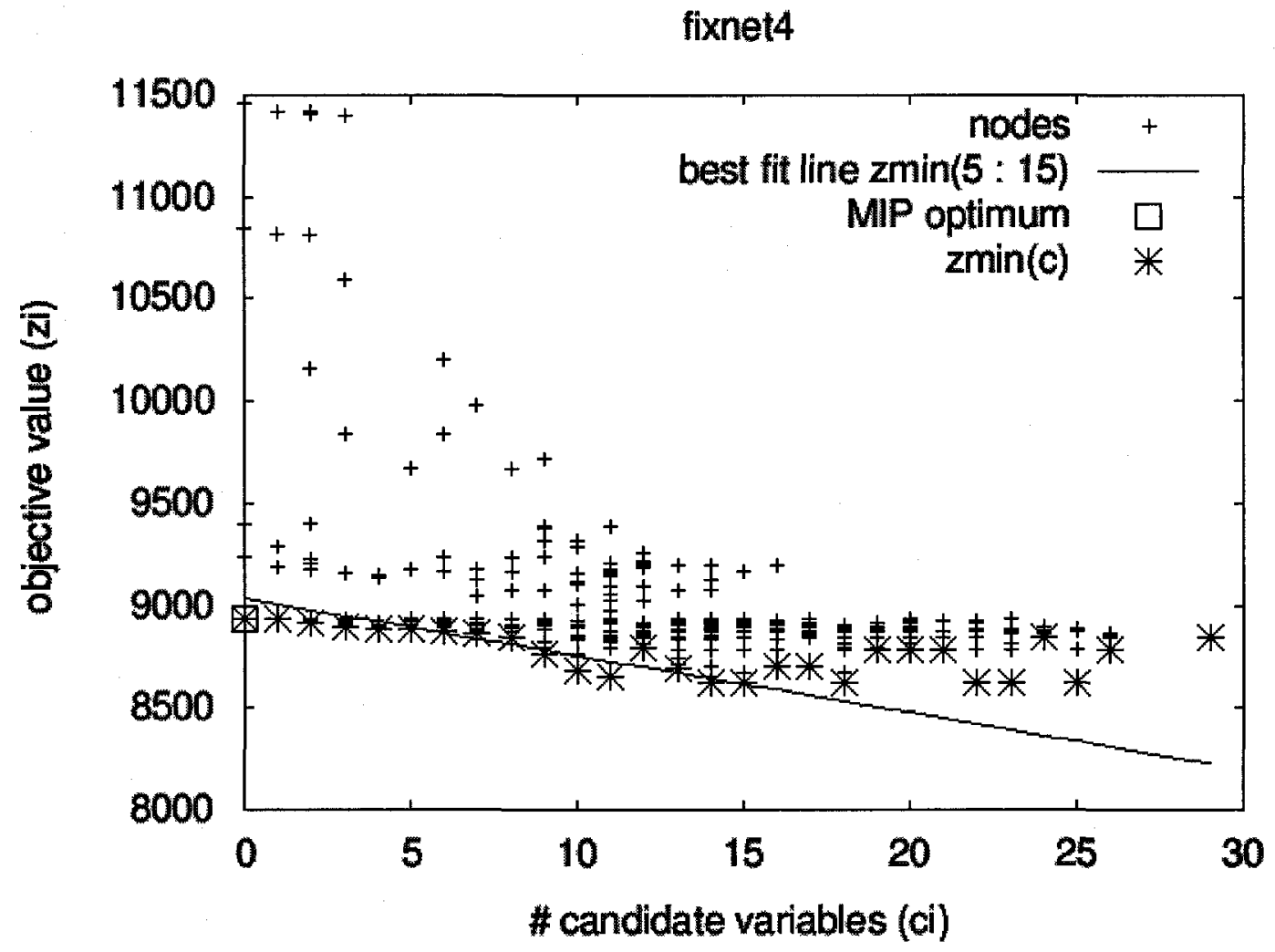

Figure 5.2: Plot of $z_{i}$ and $z^{\min }(c)$ versus $c_{i}$ for all of the nodes in the tree at the completion of the branch and bound process for the fixnet4 MIP instance. Also shown is the best fit line through $z^{\min }(c)$ for $5 \leq c \leq 15$. The DEBP-NONA-NOAN node selection configuration is used. 
Observation 1 reveals a potential improvement to the best-projection method described in Section 3.2.1. Recall that in the best-projection method an incumbent solution is required to compute an estimate of the degradation in objective value per unit MIP infeasibility (Equation 3.3). At any time during the branch and bound process, the incumbent solution objective value is $z^{m i n}(0)$ which is the minimum objective value found at a node with 0 candidate variables, i.e. a MIP feasible node. According to Observation 1, a useful estimate of the degradation in objective value per unit MIP infeasibility can be made using $z^{m i n}(c)$ for $c>0$ thus allowing modified best-projection node selection to proceed without an incumbent solution.

\subsubsection{Algorithm}

The following are some definitions needed for this algorithm.

$c_{\min }$ is the minimum number of candidate variables over all nodes solved so far. This value is initially equal to the number of candidate variables at the root node and is updated after each node is solved.

$\sigma_{z}$ is the standard deviation of $z_{i}$ over all nodes solved so far.

$\sigma_{c}$ is the standard deviation of $c_{i}$ over all nodes solved so far.

The estimate of degradation of the objective value per unit change in MIP infeasibility, $m$, is computed using Equation 5.2.

$$
m=\frac{z^{\min }\left(c_{\min }\right)-z_{0}}{c_{0}-c_{\min }}
$$

The estimate of the objective value of the best MIP feasible solution attainable from a node $i, \tilde{z}_{i}^{*}$, is computed using Equation 5.3.

$$
\tilde{z}_{i}^{*}=c_{i} * m+z_{i}
$$

The value of $-m$ is the slope of the line through $\left(c_{\min }, z^{\min }\left(c_{\min }\right)\right)$ and $\left(c_{0}, z_{0}\right)$. Figure 5.3 shows an example of this line at a time in the branch and bound process 


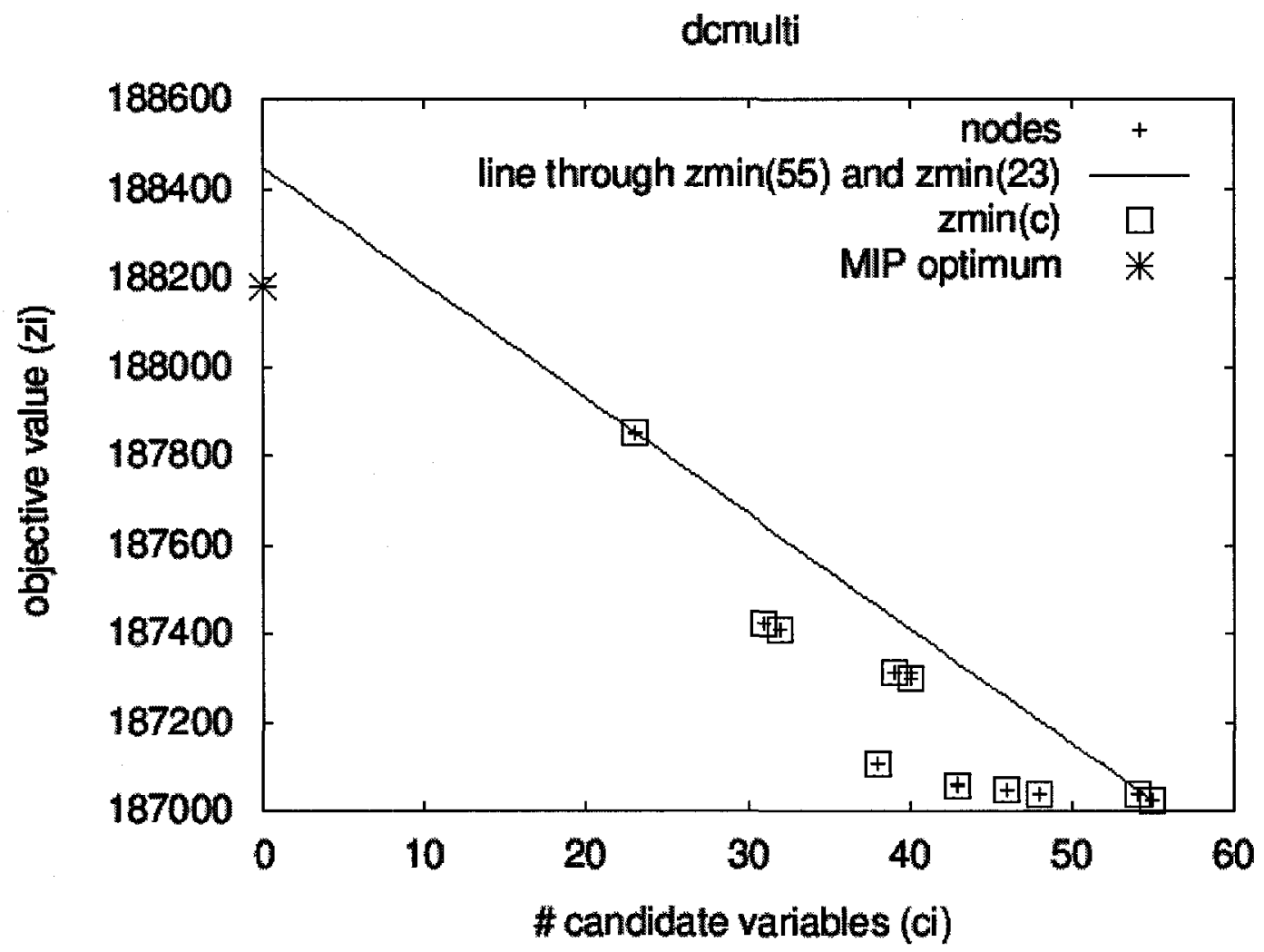

Figure 5.3: Plot of $z_{i}$ and $z^{\min }(c)$ versus $c_{i}$ for all of the nodes in the tree at the time in the branch and bound process when $c_{\min }=23$ for the $d c m u l t i$ MIP instance. Most of the points in the plot overlap because $z_{i}=z^{m i n}(c)$ for most of the nodes at this time. Also shown is the line through $z^{\min }(55)$ (the root node) and $z^{\min }(23)$.

when $c_{\min }=23, c_{0}=55$ (the number of candidate variables at the root node), $z^{\min }\left(c_{\min }\right)=187851.53$, and $z_{0}=187022.36$ (the objective value at the root node) . The slope of the line through the points $\left(c_{\min }, z^{\min }\left(c_{\min }\right)\right)$ and $\left(c_{0}, z_{0}\right)$ is $-m=-25.91$. At this time the value of $z_{i}=z^{\text {min }}\left(c_{\min }\right)$ for the most recently solved node $i$. Likewise, the value of $c_{i}=c_{\text {min }}$. An estimate of the objective value of the best MIP feasible solution attainable from node $i$, is $\tilde{z}_{i}^{*}=188447.5$. This estimate is the extrapolated value of $z^{\min }(0)$ using the line through $\left(c_{i}, z_{i}\right)$ with a slope equal to $-m$. 
If at least one of the following conditions is satisfied then this method does not work well.

- $c_{\text {min }}=c_{0}$ : In this case the denominator in Equation 5.2 is 0 .

- $\sigma_{c}<\sigma_{c}^{\min }:$ In this case most nodes have very similar values of $c_{i}$. This causes the value of $m$ to have little effect on node selection. $\sigma_{c}^{m i n}=3$.

- $\sigma_{z}<\sigma_{z}^{\min }:$ In this case there is very little change in $z_{i}$ over all nodes. This causes the value of $m$ to be almost 0 which then results in $c_{i}$ having little effect on node selection. $\sigma_{z}^{\min }=0.001$.

The thresholds used for the last two conditions were determined empirically. Some of the MIP instances for which the modified best-projection did not work well satisfied at least one of these conditions whereas this was not the case for MIP instances for which it did work well.

The modified best-projection backtracking node selection method is summarized in Algorithm 2. This algorithm is performed each time that backtracking is triggered.

Algorithm 2 (Modified best-projection backtracking node selection)

Input:

A list of active nodes $L_{u}$ in the branch and bound tree.

$\sigma_{c}^{\min }=3$.

$\sigma_{z}^{\min }=0.001$

Procedure (performed each time that backtracking is triggered):

1. If $c_{\text {min }}=c_{0}$ or $\sigma_{c}<\sigma_{c}^{\text {min }}$ or $\sigma_{z}<\sigma_{z}^{\text {min }}$ then use the best-projection backtracking node selection method described in Section 3.2.1.

2. Otherwise:

(a) For every node $i$ in $L_{u}$, compute $\tilde{z}_{i}^{*}$ using Equation 5.3.

(b) Select the node $i$ with the smallest value of $\tilde{z}_{i}^{*}$ as the node to be explored next. 


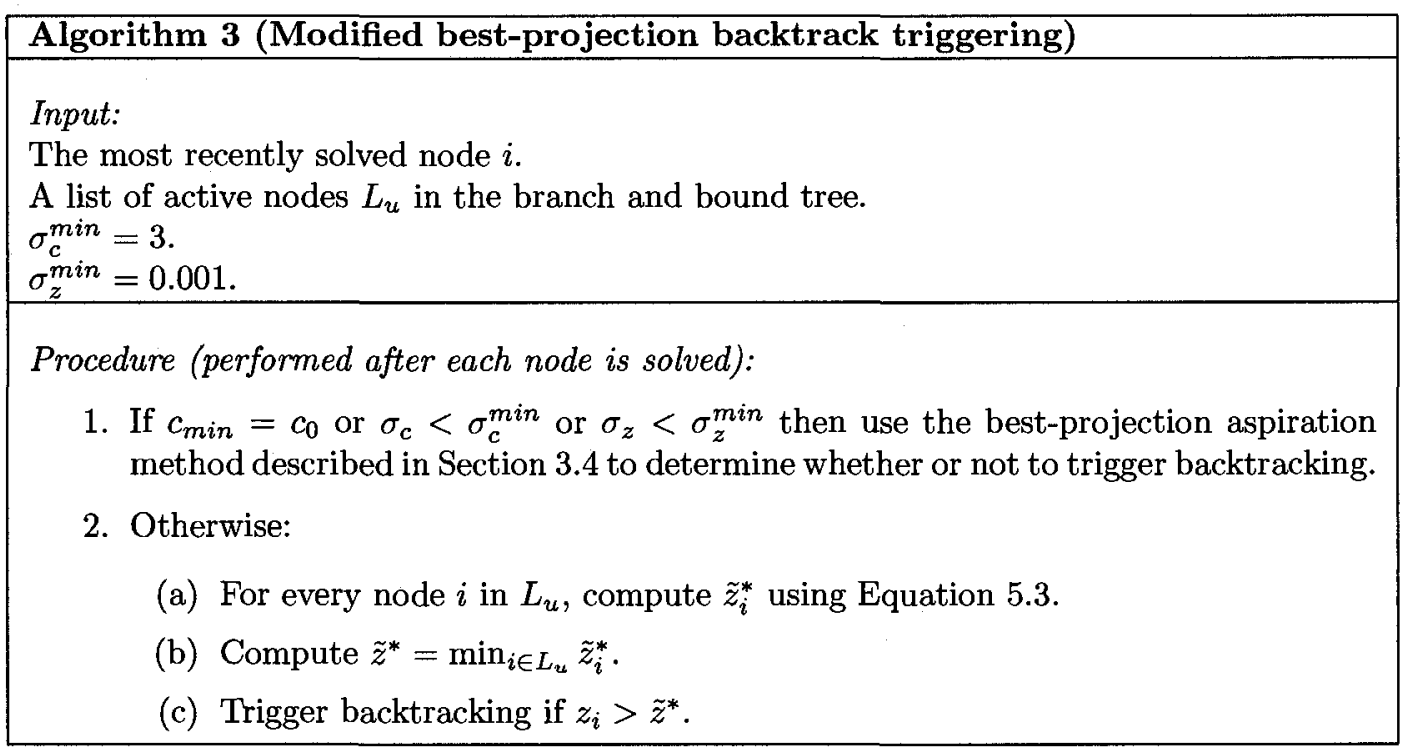

The modified best-projection backtrack triggering method is summarized in Algorithm 3. This algorithm is performed after each node is solved. 


\subsection{Distribution Node Selection}

This backtracking node selection method balances the goals of MIP feasibility and MIP optimality by using probability distributions to model these measures using all nodes solved so far in the solution process. This method weights the MIP feasibility and optimality goals dynamically so that neither can dominate the other for too long. It favours choosing nodes with lower objective values and lower MIP infeasibility.

The most commonly used measure of a node's closeness to optimality is its value of $z$. The measure of MIP infeasibility used in this method is the number of candidate variables $c$. The number of candidate variables is used instead of the sum of the fractional components of the integer variables because a candidate variable will likely become integer valued if that variable is branched on regardless of what its fractional value is. For example, suppose one node has one candidate variable with a fractional value of 0.5 whereas another node has five candidate variables each with a fractional value of 0.09. If all other integer variables are fixed for both of these nodes, then choosing the first node will likely lead to a MIP feasible node more quickly than choosing the second node, which has a lower sum of integer infeasibilities.

Observation 2 (Balancing MIP Feasibility and Optimality) A characteristic that is typical of many branch and bound trees is that there is a negative correlation between the objective value and the number of candidate variables of a node; i.e. $z_{i}$ tends to increase with depth while $c_{i}$ tends to decrease with depth.

A consequence of Observation 2 is that when trying to balance MIP feasibility and MIP optimality during node selection, if greater weight is being given to $z_{i}$ than to $c_{i}$, then the fraction of nodes chosen with small $z_{i}$ will likely be large relative to the fraction of nodes chosen with small $c_{i}$. The opposite is likely true if greater weight is given to $c_{i}$ than to $z_{i}$. Note that MIP feasibility is reached when $c_{i}=0$.

Since it is desireable to choose nodes with both a small $z_{i}$ and a small $c_{i}$, these values need to be combined into one value in order to rank all of the active nodes. This combined value should be directly proportional to both $z_{i}$ and $c_{i}$. Combining 
$z_{i}$ and $c_{i}$ in such a way that neither value dominates the other can be difficult due to the difference between the range of $z_{i}$ and the range of $c_{i}$ across all of the nodes. For example, Figure 5.1 shows that the range of $z_{i}$ for dcmulti is over 3000 , whereas the range of $c_{i}$ is less than 60 . This problem is solved by assembling the probability distributions of $z_{i}$ and $c_{i}$, respectively, as data becomes available during the solution process. Note that the range of a probability distribution function is $[0,1]$ and that each distribution is a monotonically increasing function of its independent variable.

The choice of the distribution function that is used in this method is suggested by empirical experiments which showed that using the Normal distribution function solved MIPs more quickly than the other standard distribution functions that were tested: the Uniform and Rayleigh distributions. The Normal distribution is well suited for balancing the pursuit of MIP feasibility with optimality since (i) it requires very little extra work to compute than the Uniform distribution, (ii) it estimates the values of $c_{i}$ and $z_{i}$ that most frequently appear in the tree using their respective means, and (iii) it uses information on the ranges of $c_{i}$ and $z_{i}$ via their respective standard deviations.

The means and standard deviations used to compute the Normal distributions of $z_{i}$ and $c_{i}$ are defined below.

$m_{z}=$ the arithmetic mean of $z_{i}$ over all solved nodes.

$m_{c}=$ the arithmetic mean of $c_{i}$ over all solved nodes.

$\sigma_{z}=$ the biased maximum likelihood estimate of the standard deviation of $z_{i}$ over all solved nodes.

$\sigma_{c}=$ the biased maximum likelihood estimate of the standard deviation of $c_{i}$ over all solved nodes.

Let $F_{Z}(z)=P(Z \leq z)$ and $F_{C}(c)=P(C \leq c)$ be estimates of the Normal cumulative distribution functions for $z_{i}$ and $c_{i}$, respectively, of all of the nodes solved so far. The value of $F_{Z}\left(z_{i}\right)$ is the estimated fraction of nodes with a value of $z \leq z_{i}$. 
Similarly, the value of $F_{C}\left(c_{i}\right)$ is the estimated fraction of nodes with a value of $c \leq c_{i}$. The distribution backtracking node selection method chooses the active node with the smallest value of $B_{z c}\left(z_{i}, c_{i}\right)=F_{Z}\left(z_{i}\right) \times F_{C}\left(c_{i}\right)$ to be explored next. $B_{z c}\left(z_{i}, c_{i}\right)$ is used as the comparison criterion because its value is directly proportional to both $F_{Z}\left(z_{i}\right)$ and $F_{C}\left(c_{i}\right)$, and $B_{z c}\left(z_{i}, c_{i}\right) \leq \min \left(F_{Z}\left(z_{i}\right), F_{C}\left(c_{i}\right)\right)$ (recall that $0 \leq F_{Z}\left(z_{i}\right) \leq 1$, and $\left.0 \leq F_{C}\left(c_{i}\right) \leq 1\right)$ which causes this node selection method to behave a little like a combination of the best-first and the most-feasible node selection methods.

The following example demonstrates how the pursuit of MIP feasibility and optimality can be balanced using Normal distributions. Suppose that during backtracking node selection there are 4 LP feasible nodes in the tree, two of which have one unsolved child each. The values of $z_{i}$ and $c_{i}$ for each LP feasible node are given below.

\begin{tabular}{c|c|c|c}
$i$ & $z_{i}$ & $c_{i}$ & child \\
\hline 0 & 1 & 100 & $\mathrm{n} 1$ \\
1 & 100 & 1 & \\
2 & 100 & 1 & \\
3 & 100 & 1 & $\mathrm{n} 2$
\end{tabular}

In this example, one of the candidate nodes is an unsolved child $n 1$ of node 0 , the other an unsolved child $n 2$ of node 3 . Recall that the value of $z_{i}$ and $c_{i}$ at an unsolved node is initially set to its parent's value of $z_{i}$ and $c_{i}$ respectively. The choice of which node to solve next is between node $n 1$ which has a small value of $z_{i}$ and a large value of $c_{i}$, and node $n 2$ which has a large value of $z_{i}$ and a small value of $c_{i}$. In order to achieve the goals of MIP feasibility and optimality, we would like to choose the node $i$ that has the smallest fraction of nodes with $z \leq z_{i}$ on the one hand, and the smallest fraction of nodes with $c \leq c_{i}$ on the other hand. Estimates of these fractions are computed for our example using $F_{Z}\left(z_{i}\right)$ and $F_{C}\left(c_{i}\right)$, and are given below as well as the product of these fractions $B_{z c}\left(z_{i}, c_{i}\right)$ for each candidate node.

\begin{tabular}{c|c|c|c}
$i$ & $F_{Z}\left(z_{i}\right)$ & $F_{C}\left(c_{i}\right)$ & $B_{z c}\left(z_{i}, c_{i}\right)$ \\
\hline$n 1$ & 0.067 & 0.933 & 0.062 \\
$n 2$ & 0.691 & 0.309 & 0.213
\end{tabular}


The node $n 1$ has the smallest value of $B_{z c}\left(z_{i}, c_{i}\right)$ so it is chosen to be solved next. Node $n 1$ is likely the better choice because three of the above nodes have a large value of $z_{i}$ and a small value of $c_{i}$ whereas one of the above nodes has a small value of $z_{i}$ and a large value of $c_{i}$. This indicates that more emphasis has been placed on the pursuit of MIP feasibility than the pursuit of optimality so the emphasis should be shifted towards the pursuit of optimality by selecting node $n 1$ to be solved next.

\subsubsection{Algorithm}

The distribution backtracking node selection method proceeds by computing $B_{z c}\left(z_{i}, c_{i}\right)$ for each active node $i$. The node $n$ that will be explored next is chosen using Equation 5.4 .

$$
n=\arg \min _{i} B_{z c}\left(z_{i}, c_{i}\right)
$$

If there is very little variation in the value of $z_{i}$, or if $c_{i}$ does not change significantly in proportion to the depth of the nodes in the tree, then this method does not work well since either of these measures will be given too much weight in node selection. This is summarized in the following two conditions where $d$ is the depth of the latest solved node before backtracking node selection.

- $\sigma_{c} / d<\sigma_{c / d}^{\min }:$ In this case most nodes have very similar values of $c_{i}$ over a large range of depths in the tree. $\sigma_{c / d}^{\min }=0.1$.

- $\sigma_{z}<\sigma_{z}^{\min }$ : In this case there is very little change in $z_{i}$ over all nodes. $\sigma_{z}^{\min }=$ 0.001 .

The thresholds used for these conditions were determined empirically. Some MIP instances for which distribution node selection did not work well satisfied either of these conditions whereas this was not the case for MIP instances for which it did work well.

The distribution backtracking node selection method is summarized in Algorithm 4. This algorithm is performed each time that backtracking is triggered. 
Algorithm 4 (Distribution backtracking node selection)

Input:

The most recently solved node.

A list of active nodes $L_{u}$ in the branch and bound tree.

$\sigma_{c / d}^{\min }=0.1$.

$\sigma_{z}^{m i n}=0.001$

Procedure (performed each time that backtracking is triggered):

1. If $\sigma_{c} / d<\sigma_{c / d}^{m i n}$ or $\sigma_{z}<\sigma_{z}^{m i n}$ then use the best-projection backtracking node selection method described in Section 3.2.1.

2. Otherwise:

(a) For every node $i$ in $L_{u}$, compute $B_{z c}\left(z_{i}, c_{i}\right)$.

(b) Select the node $i$ with the smallest value of $B_{z c}\left(z_{i}, c_{i}\right)$ as the node to be explored next. 


\subsection{Feasibility Depth Extrapolation Aspiration}

This method of estimating the MIP optimal objective value computes its estimate using the objective values of nodes at the estimated depth of a MIP optimal node. The depth of a MIP optimal node is estimated using linear extrapolation of the number of candidate variables along the ancestors of each node in the branch and bound tree. This method is based on Observations 3, 4 and 5 .

Observation 3 (MIP Optimal Depth and Objective Value) The largest objective value over all nodes at the depth of a MIP optimal node in the branch and bound tree is greater than or equal to the MIP optimal objective value.

Observation 4 (Relative Depth of MIP Optimal Node) The depth of a MIP optimal node in the branch and bound tree tends to be closer to the root node than the depth of most other MIP feasible nodes.

Observation 5 (Node Infeasibility versus Depth) Extrapolating the rate of decrease of the number of candidate variables along a dive in the branch and bound tree provides a reasonable estimate of the depth of the first MIP feasible solution for the dive.

Figure 5.4 shows an example that demonstrates Observation 3. In this example, the largest objective value over all nodes at a MIP optimal depth is less than the objective values of a significant number of nodes in the tree for the ran10x10a MIP instance. Therefore, if the largest objective value over all nodes at the MIP optimal depth was known and was used as an aspiration value, then the amount of effort required to solve this MIP instance would be reduced. Figure 5.4 also shows that nodes that are close to a MIP optimal depth tend to have objective values that are relatively close to the MIP optimal objective value. This suggests a method of estimating a suitable aspiration value. Finally, Figure 5.4 shows that in some instances the estimate of the depth of the optimal node does not have to be very accurate in order to get a good aspiration value. 


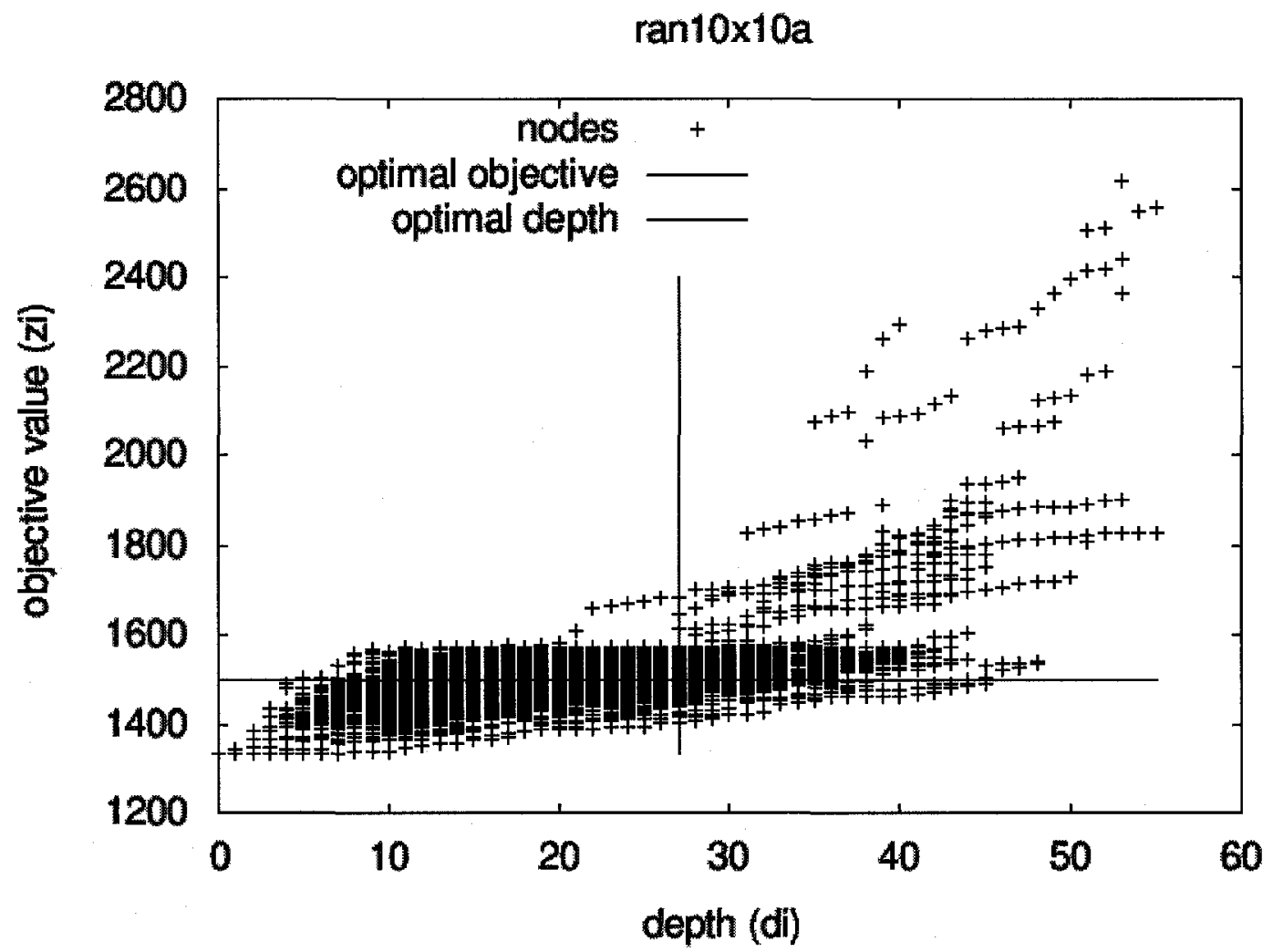

Figure 5.4: A plot of the objective value versus depth for all nodes for the ran10x10a MIP instance. Also shown are lines representing the MIP optimal objective value and depth. The DIST-NONA-NOAN node selection configuration is used. 
To demonstrate Observation 4, all MIP instances listed in Table A.1 were solved to optimality, using default GLPK with a 1 hour time limit, recording the depth of the optimal node as well as the depth of the closest MIP feasible node to the root node. Figure 5.5 shows that for a majority of MIP instances, the optimal node is close to the shallowest MIP feasible node in the branch and bound tree.

This tendency is because fewer changes in variable bounds between the initial LP relaxation and a MIP feasible solution usually lead to less change in objective values between the two solutions. Hence a shallower MIP feasible solution tends to have a better objective function value, so the shallowest MIP feasible solution is often the optimum. The rate of increase of MIP feasibility with depth tends to be greater for the ancestors of the optimal node than for other MIP feasible nodes.

If the depth $d_{i}^{*}$ of the shallowest MIP feasible node attainable by exploring the descendants of node $i$ is known, then the shallowest of these depths over all nodes in the tree is likely a MIP optimal depth. According to Observation 5, an estimate of the value of $d_{i}^{*}, \tilde{d}_{i}^{*}$, may be computed using the correlation between the number of candidate variables $c_{i}$ and the depth $d_{i}$.

Figures 5.6, 5.7 and 5.8 show examples in which changes in the number of candidate variables as one progresses along a path deeper into the tree give some indication of the depth of a MIP feasible node at the end of this path. Any node in the branch and bound tree is likely more MIP feasible than its parent because branching on a candidate variable in the parent node almost always results in that variable being integer valued in the resulting child node. Thus, it is expected that MIP infeasibility should decrease as one moves along a path deeper into the tree.

For most MIP instances, plots of $c_{i}$ versus $d_{i}$ of all nodes on a given path show some linearity. Figures 5.9, 5.10 and 5.11 show three examples of these plots for the 10teams, bell5, and vpm2 MIP instances respectively. These plots also show that extrapolating a linear best-fit line on $c_{i}$ for $0 \leq d_{i}<d_{i}^{*}$ can result in a good value of $\tilde{d}_{i}^{*}$. It should be noted that this pattern does not hold for all MIP instances so this method may not work well in some cases. Linear extrapolation is used to estimate 


\section{MIP Feasible Depth Ratios}

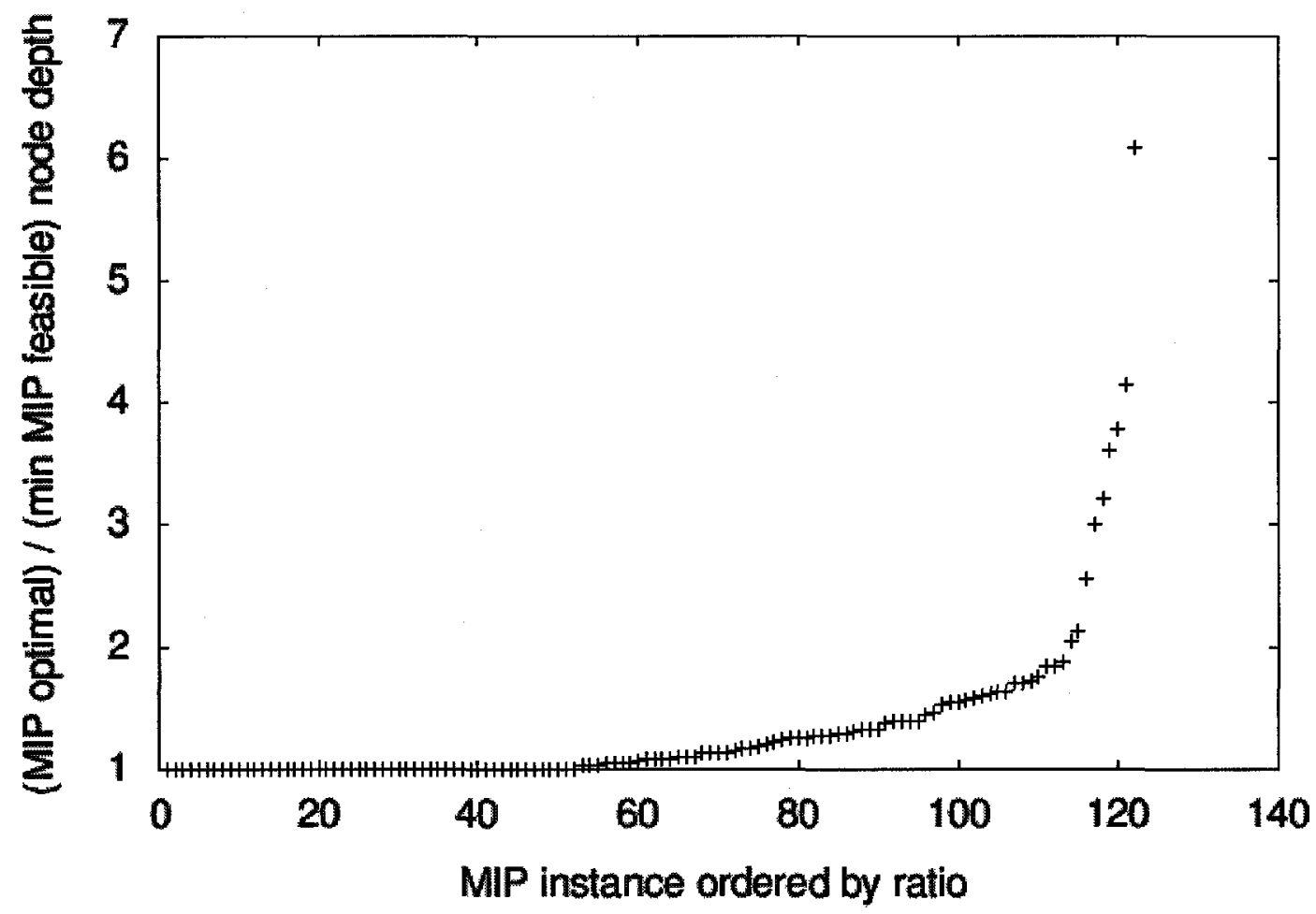

Figure 5.5: A plot of the ratio of depth of a MIP optimal node to the minimum depth over all MIP feasible nodes for a set of MIP instances. The DEBP-NONA-NOAN node selection configuration is used. 


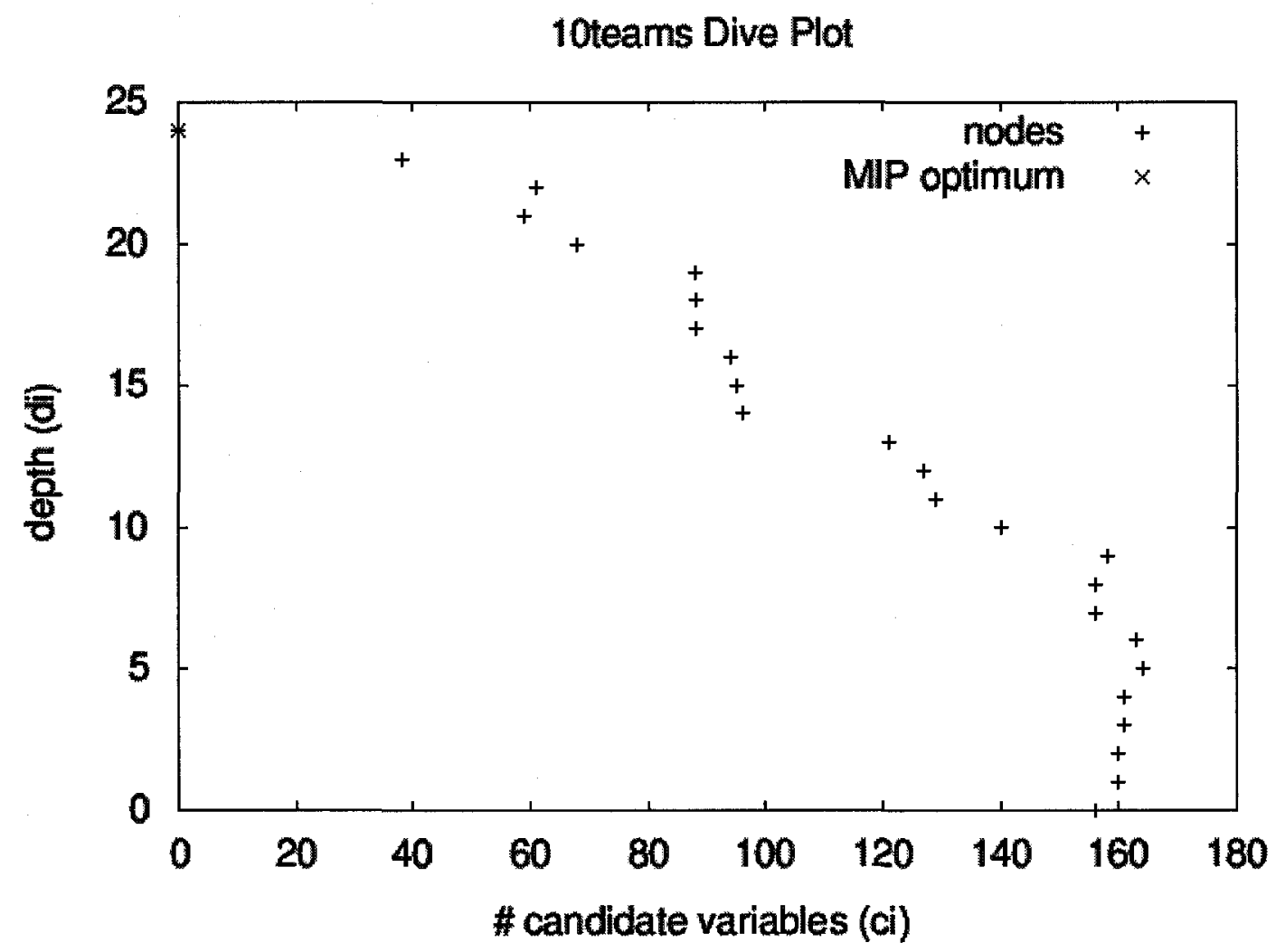

Figure 5.6: A plot of the number of candidate variables versus depth for all ancestors of a MIP optimal node for the 10teams MIP instance. 


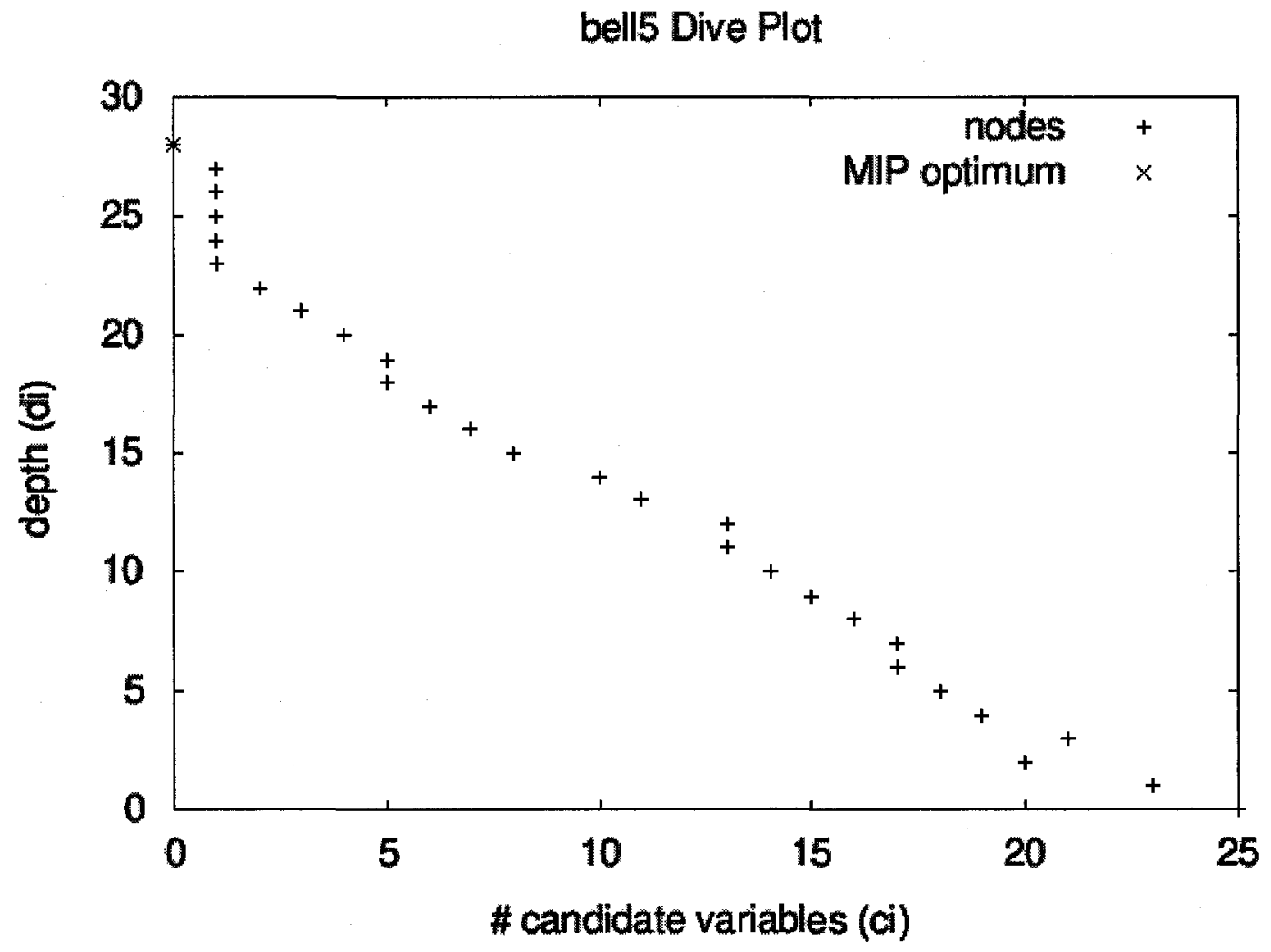

Figure 5.7: A plot of the number of candidate variables versus depth for all ancestors of a MIP optimal node for the bell5 MIP instance. 


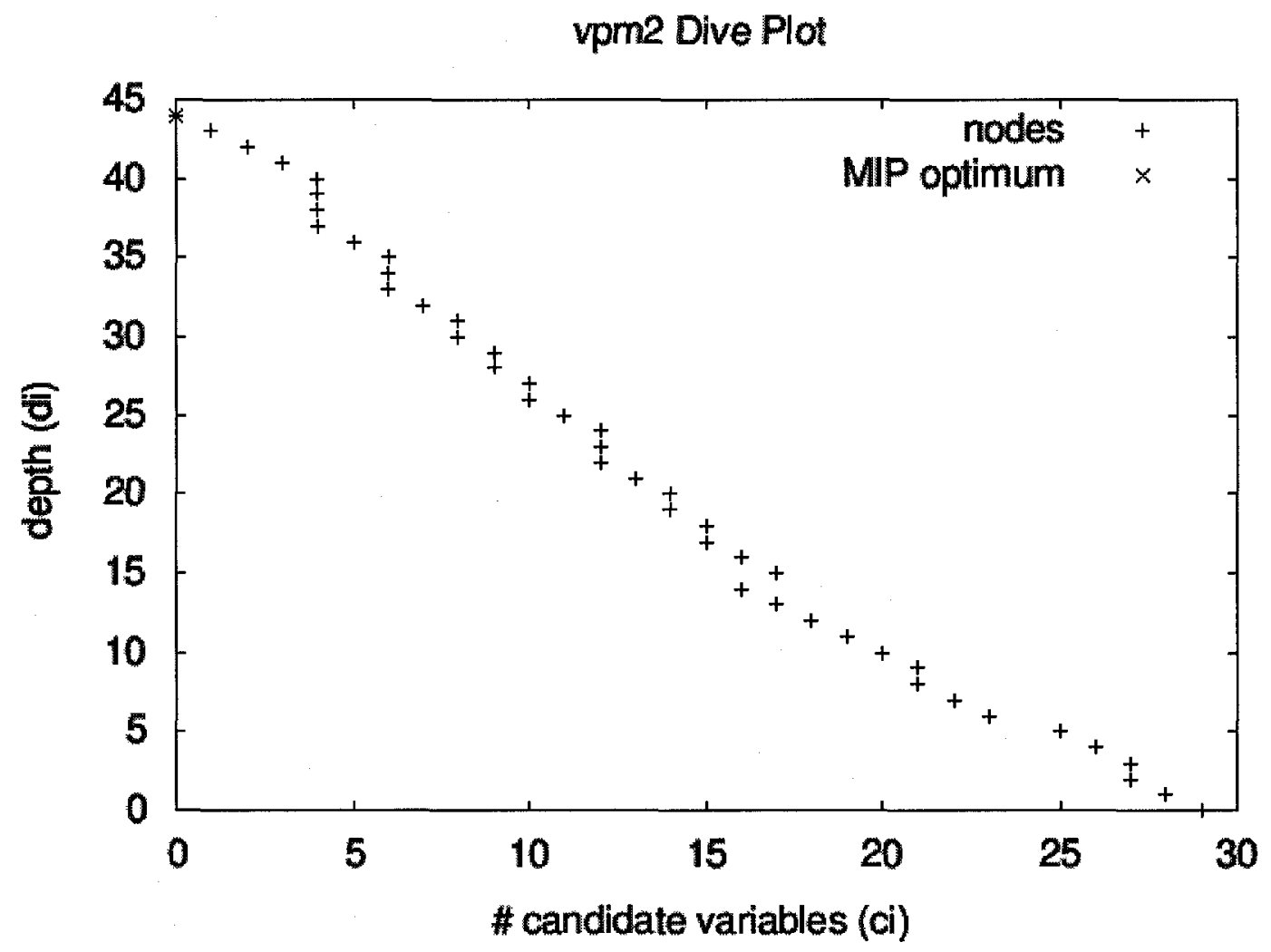

Figure 5.8: A plot of the number of candidate variables versus depth for all ancestors of a MIP optimal node for the vpm2 MIP instance. 
the value of $\tilde{d}_{i}^{*}$ of a given node $i$, i.e. the depth at which the number of candidate variables reaches zero. Figure 5.10 shows that $d_{i}^{*}=28$ and $\tilde{d}_{i}^{*}-d_{i}^{*}=-4$ for the node at $d_{i}=20$. Figure 5.11 shows that $d_{i}^{*}=44$ and $\tilde{d}_{i}^{*}-d_{i}^{*}=-8$ for the node at $d_{i}=20$. Figure 5.9, on the other hand, shows how the value of $d_{i}^{*}$ can be much too large. In this case $d_{i}^{*}=24$ and $\tilde{d}_{i}^{*}-d_{i}^{*}=12$ for the node at $d_{i}=20$. An estimate of the depth of the optimal solution $\tilde{d}^{*}$, according to Observation 4 , is then taken as the smallest $\tilde{d}_{i}^{*}$ over all solved nodes in the tree.

The aspiration value $\tilde{z}^{*}$, according to Observation 3 is set to the maximum objective value over all solved nodes at $\tilde{d}^{*}$.

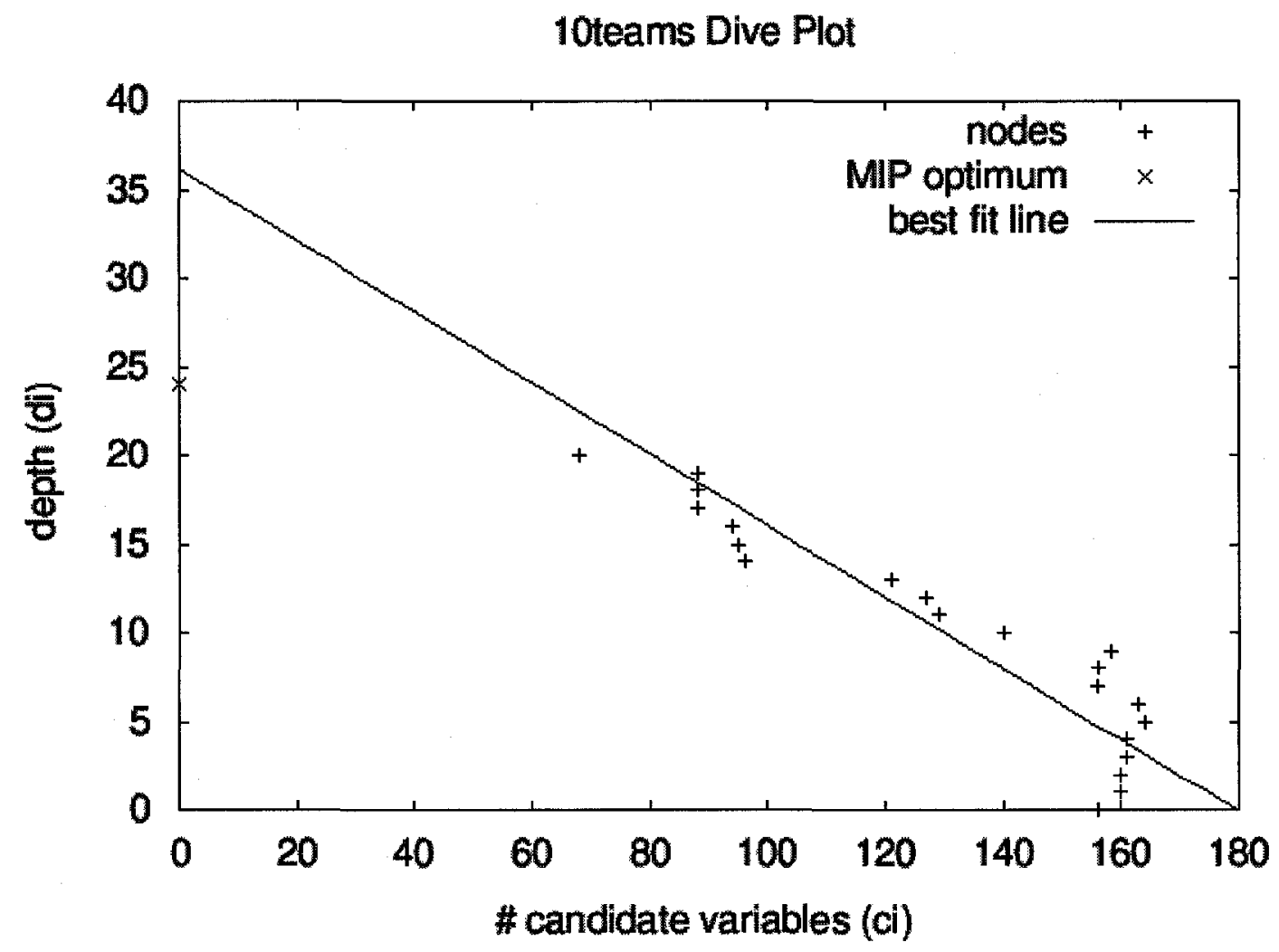

Figure 5.9: A plot of the number of candidate variables versus depth for the earliest 20 ancestors of a MIP optimal node for the 10teams MIP instance along with the best fit line. 


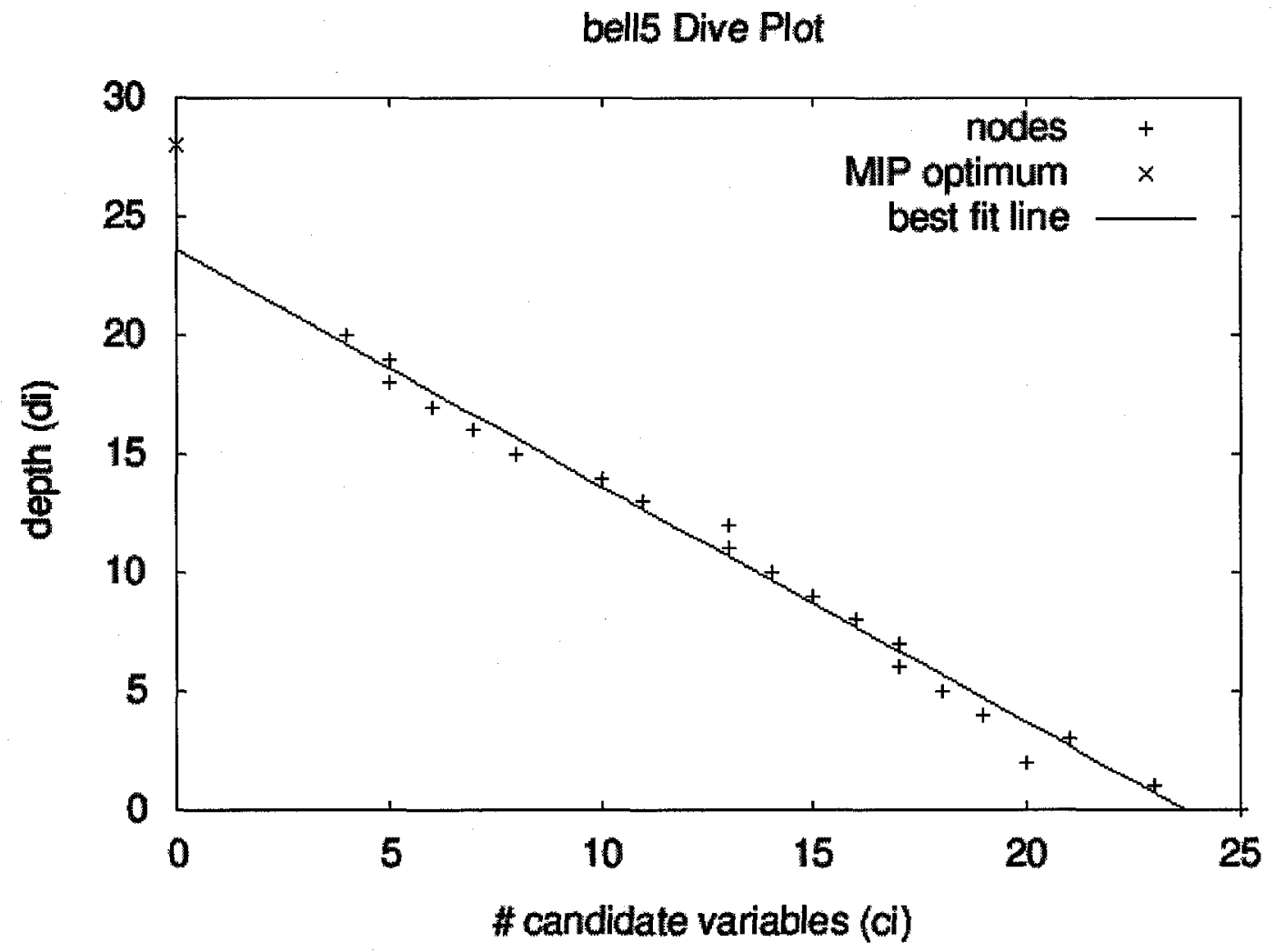

Figure 5.10: A plot of the number of candidate variables versus depth for the earliest 20 ancestors of a MIP optimal node for the bell5 MIP instance along with the best fit line. 


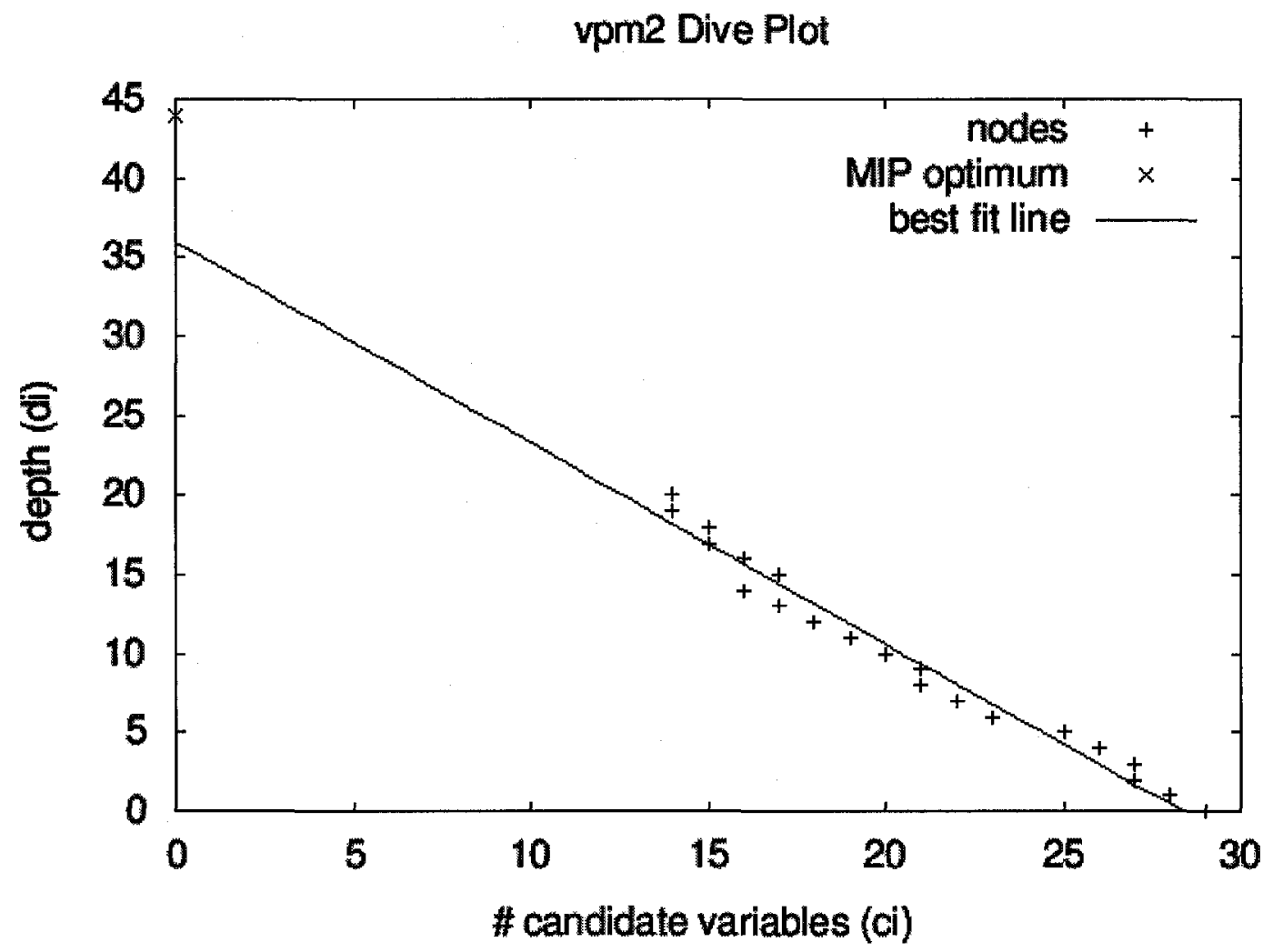

Figure 5.11: A plot of the number of candidate variables versus depth for the earliest 20 ancestors of a MIP optimal node for the vpm2 MIP instance along with the best fit line. 


\subsubsection{Algorithm}

The following are some definitions needed for this algorithm.

$m_{i}$ is the slope of the least squares best fit line for $\left(c_{i}, d_{i}\right)$ over all ancestors of node $i$.

$b_{i}$ is the $d$-intercept of the least squares best fit line $\left(d=m_{i} \cdot c+b_{i}\right)$ over all ancestors of node $i$.

$S$ is the set of solved nodes in the current branch and bound tree that are LP feasible but not MIP feasible.

The algorithm proceeds by computing the least squares best fit line of the ancestors for each solved node with at least 20 ancestors. The condition that a node have at least 20 ancestors is imposed to ensure enough information is present to compute a reasonably accurate best fit line while still being able to compute an aspiration value early in the branch and bound process. An estimate of the depth of the first MIP feasible solution attainable from a node $i$ is computed as

$$
\tilde{d}_{i}^{*}=\left[b_{i}\right]
$$

where $\left[b_{i}\right]$ represents the rounding of $b_{i}$ to the nearest integer value.

The estimate of the optimal solution depth, $\tilde{d}^{*}$, is computed as

$$
\tilde{d}^{*}=\min _{\left\{i \in S: d_{i} \geq 20, \tilde{d}_{i}^{*}>0\right\}} \tilde{d}_{i}^{*}
$$

The aspiration value $\tilde{z}^{*}$, according to Observation 3 , is the maximum value of $z_{i}$ over all solved nodes with $d_{i}=\tilde{d}^{*}$.

$$
\tilde{z}^{*}=\max _{i \in S: d_{i}=\tilde{d}^{*}} z_{i}
$$

The condition that $\tilde{d}_{i}^{*}>0$ is imposed in Equation 5.6 because nonpositive values would result in unreasonable values of $\tilde{d}^{*}$. A value of $\tilde{d}^{*}=0$ is unreasonable since the 
root node is not MIP feasible. A value of $\tilde{d}^{*}<0$ is unreasonable since there are no nodes with $d_{i}<0$.

The feasibility depth extrapolation backtrack triggering method is summarized in Algorithm 5. This algorithm is performed after each node is solved.

Algorithm 5 (Feasibility depth extrapolation backtrack triggering)

Input:

The most recently solved node $i$.

The set $S$ of solved nodes that are LP feasible but not MIP feasible in the current branch and bound tree.

The threshold on the minimum node depth $d^{\text {min }}=20$.

The current value of $\tilde{d}^{*}$.

The current value of $\tilde{z}^{*}$.

Procedure (performed after each node is solved):

1. If $d_{i}=0$ then the current node is the root node so set $\tilde{d}^{*}=\infty$ and $\tilde{z}^{*}=\infty$.

2. If $d_{i} \geq d^{\min }$ :

(a) Compute $\tilde{d}_{i}^{*}$ using Equation 5.5 .

(b) If $\tilde{d}_{i}^{*}>0$ and $\tilde{d}_{i}^{*}<\tilde{d}^{*}$ then set $\tilde{d}^{*}=\tilde{d}_{i}^{*}$;

3. If $\tilde{d}^{*}<\infty$ then update the value of $\tilde{z}^{*}$ using Equation 5.7 .

4. Trigger backtracking if $z_{i}>\tilde{z}^{*}$. 


\subsection{Active Node Search Threshold}

Some backtracking node selection methods can be computationally expensive. The active node search threshold (ANST) estimates whether or not the cost of using a backtracking node selection method outweighs its benefit. If the cost of a method does outweigh its benefit, then a simpler node selection method should be used instead. This method is based on Observation 6 .

Observation 6 (Cost of Node Selection Methods) For some MIPs, the amount of time required to perform a given backtracking node selection method can become a significant fraction of the overall amount of time required to solve it. This occurs when the number of active nodes in the tree becomes very large.

The MIP instance mas 76 , for example, is solved in 20609 seconds, requiring 3186117 simplex iterations and 1177063 nodes, using best-projection backtracking node selection. The same MIP instance is solved in 1306 seconds, requiring 3314480 simplex iterations and 1229699 nodes using depth-first node selection. This MIP is solved in significantly less time but requiring more simplex iterations and nodes using depth-first versus best-projection.

To verify that the extra time needed to solve MIPs such as mas76 using bestprojection versus depth-first backtracking is due to the node selection method, the maximum ratio, $R_{t}$, of total time spent performing all of the best-projection backtracking node selections to the total time spent performing all of the other branch and bound operations was recorded for the experiments reported in this thesis. For a small set of MIP instances, the amount of effort needed to solve each MIP instance is shown in Table 5.1 along with the corresponding value of $R_{t}$. The value of $R_{t}$ for MIPs that are solved in less time but more simplex iterations using depth-first versus best-projection are much larger than the values of $R_{t}$ for the remaining MIPs.

For the $\operatorname{mas} 76, p k 1$, and $\operatorname{ran} 10 x 10 c$ MIP instances, the cost of using best-projection node selection outweighs the benefit because this method searches the entire set of active nodes and the number of active nodes in the tree becomes very large. The 
cost of using a simple backtracking node selection method does not increase with the number of active nodes in the tree. In GLPK [27], the depth-first backtracking node selection method is simple and requires very little computation because it chooses the active node that was last created and resides at the end of the list of active nodes. A backtracking node selection method that searches through the entire set of active nodes is not simple because the amount of effort required to search through a set increases as the number of nodes in the set increases. Examples of backtracking node selection methods that are not simple include the best-projection, best-first, and best-estimate methods. 


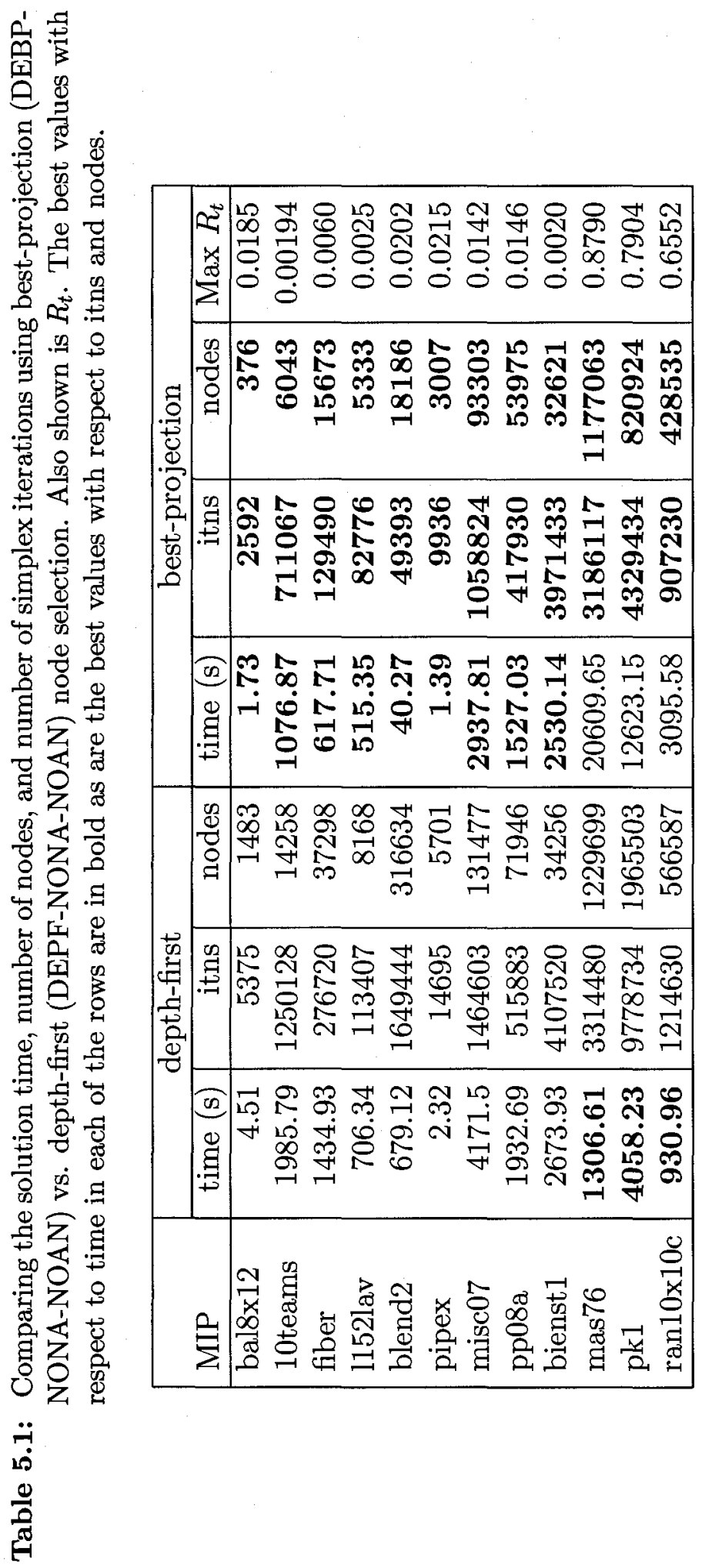


An alternative to putting the last created active node at the end of the list is used by Achterberg [3, 4] in the implementation of SCIP for solving MIPs and constraint integer programs. In SCIP, each node is placed in a list according to its priority with respect to the node selection criteria. A drawback to this method is that the relative priority of nodes already in the list may change depending on the node selection criteria used. In the best-estimate node selection method described in Section 3.2.2, for example, the pseudo-cost estimates are updated after every node is solved. So to ensure that the nodes in the list have the correct order, the best-estimate computation of each node in the list affected by the change in pseudo-cost estimates should be performed again and then all of the active nodes should be sorted.

In SCIP, the amount of computation required to place each node in the list according to its node selection priority increases with the number of nodes already in the list since a search of these nodes needs to be performed every time a new node is placed in the list. Therefore it is possible for the cost of node selection methods of this type to become very large. This issue is noted by Achterberg [3].

The cost of a backtracking node selection method is mostly affected by the number of active nodes in the tree, the amount of computation required per active node during the backtracking search, and by how frequently backtracking node-selection is performed. If an aspiration backtrack triggering method is used then backtracking frequency is affected by the aspiration value. Therefore, if the cost of backtracking node selection is perceived to outweigh its benefit for a given MIP, and an aspiration method is being used, then it may be better to first reduce the backtracking frequency by increasing or removing the aspiration value to see if backtracking continues to cost too much. If the backtracking node selection method continues to cost too much, then it should be changed to a simple method. This is one way in which an aspiration value that is too small may be detected. 


\subsubsection{Algorithm}

The following are some definitions needed for this algorithm.

$t_{N S}$ is the total amount of time spent performing all backtracking node selections.

$t_{B B}$ is the total amount of time spent performing all of the operations in the branch and bound method.

$q$ is the threshold that determines when to switch to a simple node selection method.

$$
q=0.1
$$

$d q$ is an increment that is used to determine the effect of the currently used aspiration backtrack triggering method on the cost of backtracking node selection. $d q=$ 0.01

The active node search threshold algorithm proceeds by computing the value of $R_{t}$, using Equation 5.8, after each time that backtracking node selection is performed.

$$
R_{t}=\frac{t_{N S}}{t_{B B}-t_{N S}}
$$

If the value of $R_{t} \geq q$ then either backtracking is being performed too frequently because of an aspiration backtrack triggering method, or the cost of backtracking outweighs its benefit. If no aspiration backtrack triggering method is currently being used, then the current backtracking node selection method is too costly, and depthfirst backtracking node selection replaces the current node selection method. If an aspiration backtrack triggering method is currently being used, then the use of the aspiration value is discontinued, the current backtracking node selection method continues, and the value of $q$ is set to the current value of $R_{t}+d q$ so that now $R_{t}<q$. If the value of $R_{t}$ continues to increase and exceeds this new value of $q$ then the current backtracking node selection method is too costly and the depth-first backtracking node selection method is then substituted. 
Depth-first is used as the simple backtracking node selection method because of its low cost and its good efficiency in exploring the branch and bound tree as discussed in Section 2.2.2.

Table 5.1 shows that setting $q=0.1$ allows a given node selection method to continue to be used when it is not too costly but quickly forces a change to the depth-first method when its cost becomes too great. Although the values of $R_{t}$ for a small number of MIP instances were used to determine the value of $q$, the chosen value of $q=0.1$ is over 4 times larger than the value of $R_{t}$ that is closest to $q=0.1$. The value of $d q$ is set to a small value that, if an aspiration value is used, is added to $R_{t}$ to make a new threshold that allows the current backtracking node selection method to continue to be used without an aspiration value. The value of $d q$ is set to $10 \%$ of the value of $q$ to allow the current backtracking node selection method to continue to be used a sufficient number of times without an aspiration backtrack triggering method so that the backtracking node selection method is not mistakenly determined to be too costly.

The active node search threshold (ANST) method is summarized in Algorithm 6. 
Algorithm 6 (Active Node Search Threshold)

Input:

A MIP instance using a backtracking node selection method other than depth-first.

The total amount of time spent performing all backtracking node selections, $t_{N S}=0$.

The total amount of time spent performing branch and bound, $t_{B B}=0$.

The threshold value, $q=0.1$.

The threshold increment value, $d q=0.01$.

Procedure:

1. Perform branch and bound until backtracking is triggered.

2. Perform backtracking node selection.

3. If depth-first backtracking node selection is being used then go to Step 1.

4. Add the time required to perform Steps 1 and 2 to $t_{B B}$. Add the time required to perform Step 2 to $t_{N S}$.

5. Compute the ratio of time spent backtracking to the time spent on branch and bound, $R_{t}$, using Equation 5.8.

6. If $R_{t}<q$ then go to Step 1 .

7. If no aspiration value is in use then

(a) change backtracking node selection method to depth-first.

(b) Go to Step 1.

8. $q=R_{t}+d q$.

9. Stop using aspiration value.

10. Go to Step 1. 


\section{Chapter 6}

\section{Experimental Setup}

This chapter presents details of the hardware, the software, the MIP solver parameters, the MIP instances, and the metrics that are used in the experiments that evaluate the performance of various configurations of backtracking node selection methods, aspiration methods, and active node search thresholds.

\subsection{Hardware}

The following hardware and software was used in all experiments.

CPU: Intel Core $26600 @ 2.4 \mathrm{GHz}$

RAM: 4 GB

OS: Linux 2.6.18

Although the CPU has two processing cores, each MIP instance is solved using only 1 core.

\subsection{MIP Solver}

The GLPK 4.9 [27] MIP solver was used in all experiments because: 
- it is a free open source software that has excellent documentation;

- it uses the depth-first with backtracking framework;

- it is easy to add new backtracking node selection methods, aspiration methods, and active node search thresholds to it;

- as shown in Table 3.1, it has most of the standard node selection methods;

- and it is capable of solving reasonably difficult MIP instances within a reasonable time limit [25].

The GLPK solver has many parameters that do not affect the branch and bound solution method. Unless otherwise stated, all of these parameters are set to their default values except for those described below.

LPX_K_TMLIM: The time limit of the MIP solver in seconds.

LPX_K_MSGLEV: The type of messages the solver outputs to the console.

For all experiments the value of LPX_K_MSGLEV is set to 1 so that messages to the console are limited to error messages in order to reduce the corresponding overhead and thus reduce the solution time for all MIP instances.

\subsection{Performance Measures}

Solution time $t_{M I}$ is the most important measure of the amount of effort required to solve a MIP instance $M I$ and is frequently used to evaluate the performance of a branch and bound method $[3,8,9,2,26]$. The number of nodes solved and the number of simplex iterations are also commonly used to measure the amount of effort required to solve a MIP instance [3, 8, 14, 33, 2]. Solution time is used for the experiments in this thesis because the time to solve a MIP instance includes the overhead computing time that is not reflected in the number of nodes and the 
number of simplex iterations required to solve this MIP instance. Section 5.4 shows an example of how the time spent performing node selection may become large relative to that spent performing the other aspects of branch and bound. Table 5.1 shows that although best-projection node selection solves the mas76, pk1 and ran10x10c MIP instances in fewer nodes and simplex iterations than depth-first node selection, these MIP instances are solved in significantly less time with depth-first versus bestprojection node selection. Therefore the number of nodes and simplex iterations do not always accurately reflect the amount of effort required to solve a MIP instance.

In order to evaluate the relative performance of a set of branch and bound methods, a set of many MIP instances should be solved using each method because the heuristic nature of these methods does not guarantee that the relative performance of each method will be consistent for every possible MIP instance. A difficulty in using a set of many MIP instances to determine the relative performance of each method lies in how to combine the solution times for all of the MIP instances into a single performance measure.

A simple method of combining the solution times of all of the MIP instances is simply to compute their total using equation 6.1 .

$$
T O T T M=\sum_{M I} t_{M I}
$$

A drawback of using TOTTM is that its value can be dominated by a small number of very difficult MIP instances. The impact of a particularly difficult MIP instance on the overall performance of a method can be eliminated by using the best solution time $t_{M I B}$ for $M I$ over all of the contending methods to normalize $t_{M I}$ using Equation 6.2.

$$
r_{M I}=t_{M I} / t_{M I B}
$$

For a given method, $r_{M I}-1$ is the percentage of extra time required to solve $M I$ relative to the best method for solving $M I$. The geometric mean defined in Definition 1 is used to combine the $r_{M I}$ for every $M I$ into a single measure MRATE. The 
geometric mean is preferable to the arithmetic mean because the geometric mean is less sensitive to outlying values of $r_{M I}$ that may occur with large sets of MIP instances. For example, the arithmetic means of each of the sets $A=1,2,3,4,5$ and $B=1,1,1,1,11$ are both equal to 3 whereas the geometric mean of each set is 2.61 and 1.62 respectively. The outlying member in $B, 11$, has less impact on the geometric mean than on the arithmetic mean.

Definition 1 (Geometric Mean) Let $r_{1}, \ldots, r_{N}$ be a set of real numbers with values greater than 0. The geometric mean of this set is computed using Equation 6.3.

$$
\ddot{r}=\sqrt[N]{\prod_{j=1}^{N} r_{j}}
$$

If a time limit is imposed on the branch and bound method, then not all MIP instances will be solved in an experiment. The number of MIP instances FAIL that are not solved to optimality is a commonly used performance measure for a branch and bound method [26, 9$]$.

The details of computing F AIL, TOTTM and MRATE for a set of MIP instances are given below.

FAIL: The number of MIP instances used in an experiment that are not solved to optimality within the time limit. If a MIP instance is not solved using any contending method, then this MIP instance is excluded from $F A I L$. Methods with a smaller value of $F A I L$ are better.

TOTTM: The total amount of time required to solve the set of MIP instances. Any MIP instance that is not solved using any contending method is excluded from this total. If a MIP instance is not solved using a given contending method but is solved using at least one other contender, then the time limit is added to this total for this instance. TOTTM is, therefore, a lower bound on the total time required to solve the set of MIP instances if a method fails on at least one MIP 
instance (it is exact if the method solves all instances). Methods with a smaller value of TOTTM are better.

MRATE: The geometric mean, see Definition 1, of $t_{M I} / t_{M I B}$ over all solved MIP instances for a given contending method. $t_{M I}$ is the time required to solve a MIP instance $M I . t_{M I B}$ is the best time to solve MIP instance $M I$ over all contending methods. Any MIP instance that is not solved using any contending method is excluded from this mean. If a MIP instance is not solved using a given contending method but is solved using at least one other contender, then $t_{M I}=$ the time limit. MRATE is, therefore, a lower bound if a method fails on at least one MIP instance (it is exact if the method solves all instances). If $t_{M I}$ is smaller than 10 seconds for every contending method then $M I$ is excluded from MRATE to reduce the effects of timing error on this measure. Methods with a smaller value of MRATE are better.

Another method of combining the solution times of several MIP instances in order to evaluate the relative performance of contending methods is to use a performance profile [13]. A performance profile for a contending method is a plot that displays a point representing each $M I$ that is solved. For a given MIP instance $M I 1$ and contending method, the $\mathrm{x}$-axis represents the value of $r_{M I 1}$ and the $\mathrm{y}$-axis represents the fraction $f_{M I 1}$ of MIP instances in the set that have a value of $r_{M I} \leq r_{M I 1}$ for a particular method. Figure 7.10 shows an example of a performance profile. In this example, $r_{M I} \leq 1.5$ for a little more than $80 \%$ of MIP instances for the MOBPMPAS-ANST contender. In general, contending methods with points located nearest the top-left of the performance profile are considered to be best. By looking for points where $r_{M I}=1$, the performance profile shows the fraction of MIP instances that a contending method performs best. The largest value of $f_{M I}$ over all of the MIP instances for a contending method is the fraction of MIP instances that are solved using this method. If $t_{M I}$ is smaller than 10 seconds for every contending method then $M I$ is excluded to reduce the effects of timing error on the performance profile. 
Commonly used performance measures for unsolved MIP instances are the best incumbent solution found and proven optimality gap at the termination of the branch and bound method [26, 9]. In order to determine how the competitor methods performed on the more difficult MIP instances, the following scheme is used to determine the relative ranking of two methods for a given MIP instance. Ties are allowed.

- If both methods find a MIP feasible solution then the better method is the one that finds the solution with the best value of the objective function.

- If both methods find equally good MIP feasible solutions then the method with the smaller provable optimality gap (See Definition 2) is better.

- A method is better if it finds a MIP feasible solution whereas the other method does not.

Definition 2 (Provable optimality gap) The provable optimality gap $G_{o}$ represents the proximity of the upper and lower bounds on the MIP optimal objective value. The value of $G_{o}$ is computed as follows.

$$
G_{o}=\frac{\left|z_{i n c}-z_{g}^{m i n}\right|}{\left|z_{i n c}\right|+\epsilon}
$$

where

$z_{g}^{m i n}=$ the minimum objective value over all active nodes;

$z_{i n c}=$ the objective value of the incumbent solution;

$\epsilon=10^{-9}$ (used to avoid dividing by 0 )

$A M I P$ is solved if $G_{o}=0$.

The following measures are based on the above ranking scheme. 
AVGRANK is the average rank of a given method over all MIP instances that are not solved using any contending method. MIP instances for which no incumbent solution is found by any contending method are also excluded. Methods with a smaller value of $A V G R A N K$ are better.

NFIRSTS is the number of MIP instances for which a given method is ranked first. MIP instances that are solved by at least 1 contending method are excluded. MIP instances for which no incumbent solution is found by any contending method are also excluded. Methods with a larger value of NFIRSTS are better.

$N I N C$ is the number of MIP instances for which a given method did not find an incumbent solution. MIP instances that are solved by at least 1 contending method are excluded. MIP instances for which no incumbent solution is found by any contending method are also excluded. Methods with a smaller value of $N I N C$ are better.

\subsection{MIP Instances}

As is mentioned in Section 6.3, in order to evaluate the performance of branch and bound methods, a sufficiently large set of MIP instances should be solved using these methods. The set of MIP instances used in the experiments can have a large impact on the results. The set of MIP instances used in the experiments to determine the best node selection configuration is chosen such that MIP instances with a wide variety of MIP formulation characteristics are included because there are no restrictions placed on the new node selection methods proposed in Chapter 5 with respect to MIP formulation characteristics.

The experiments presented in this thesis use all 116 MIP instances from MIPLIB (MIPLIB 2003 [5], MIPLIB 3.0 [11], and MIPLIB 2.0 [10]) and all 156 MIP instances from CORAL [24], for a total of 272 MIP instances. The MIP instances taken from these sources are problems from industry, theoretical problems, or problems submitted 
Table 6.1: The minimum, average, and maximum values of the given formulation characteristics.

\begin{tabular}{|l|l|l|l|}
\hline & Min & Avg & Max \\
\hline Cols & 9 & 10251.27 & 236376 \\
Real & 0 & 2906.45 & 218806 \\
Int & 0 & 491.05 & 67732 \\
Bin & 0 & 6853.77 & 236376 \\
Rows & 4 & 7897.77 & 318335 \\
Ineq & 0 & 6640.47 & 318334 \\
Eq & 0 & 1256.3 & 73559 \\
NZ & 54 & 104453.55 & 3946449 \\
\hline
\end{tabular}

to the NEOS server. These MIP instances are commonly used in testing branch and bound methods [3, 25]. A complete list of these MIP instances is shown in Table A.1 along with the formulation characteristics described below. Table 6.1 shows the minimum, average, and maximum values of these formulation characteristics.

Cols: The number of variables.

Real: The number of real variables.

Int: The number of non-binary integer variables.

Bin: The number of binary variables.

Rows: The number of constraints.

Ineq: The number of inequality constraints.

Eq: The number of equality constraints.

$N Z$ : The number of non-zero constraint and objective function coefficients. 


\subsection{Branch and Bound Parameters}

Three parameters that affect the performance of the branch and bound method are described below.

- The backtracking node selection method used (GLPK parameter: LPX_K_BTRACK ) .

- The branching variable selection method used (GLPK parameter: LPX_K_BRANCH ).

- The root node heuristics used (GLPK parameter: LPX_K_USECUTS ).

\subsubsection{Branching Variable Selection Parameter}

When performing the experiments to determine the best node selection configuration to use when solving MIPs, the branching variable selection method used should be the best available. To determine the best available branching variable selection method, the set of 116 MIP instances shown in Table A.2 was solved four times, each time using a different contending method with the same settings for all other parameters. The contending branching variable selection methods are described below.

BFIRST: Branch on the first candidate in the list of variables.

BLAST: Branch on the last candidate in the list of variables.

BDRTO: Branch on the candidate variable selected using the method by Driebeek [14] and Tomlin [38] described in Section 2.2.1.

BMFRAC: Branch on the candidate variable with the fractional value closest to 0.5 .

These methods are used because they are the only methods available in GLPK. A commonly used state of the art branching variable selection method that uses pseudocosts is not implemented in GLPK and is thus excluded from the list of contending methods. 
Table 6.2: Performance of contending branching variable selection methods. The best values in each of the FAIL, TOTTM and MRATE columns are in bold.

\begin{tabular}{|l|r|r|r|}
\hline Contender & FAIL & TOTTM & MRATE \\
\hline BFIRST & 8 & 33543.63 & 2.75 \\
BLAST & 11 & 46272.01 & 3.93 \\
BDRTO & $\mathbf{4}$ & $\mathbf{2 4 9 6 6 . 1 8}$ & $\mathbf{2 . 0 9}$ \\
BMFRAC & 8 & 41363.03 & 3.49 \\
\hline
\end{tabular}

The values of the GLPK parameters not involving branching variable selection are set as follows.

- The best-projection backtracking node selection method (Section 3.2.1) is used. This is the default method in GLPK (LPX_K_BTRACK $=2$ ).

- Do not use Gomory's mixed integer cuts at the root node. This is the default setting in GLPK (LPX_K_USECUTS $=0$ ).

- A time limit of 1 hour is used (LPX_K_TMLIM $=3600$ seconds).

The results of this experiment are summarized in Table 6.2. 64 MIP instances were solved by at least 1 contending method. The BDRTO method is clearly the best of the contending methods and therefore is used in all subsequent node selection experiments. Note that BDRTO is the default method in GLPK. The complete results of using BFIRST, BLAST, BDRTO and BMFRAC in this experiment are shown in Appendix B.1. 
Table 6.3: Performance of root node cuts vs no cuts. The best values in each of the FAIL, TOTTM and MRATE columns are in bold.

\begin{tabular}{|l|r|r|r|}
\hline Contender & FAIL & TOTTM & MRATE \\
\hline NOCUTS & 5 & 28567.19 & 5.85 \\
CUTS & $\mathbf{3}$ & $\mathbf{1 6 7 4 0 . 1 5}$ & $\mathbf{5 . 0 8}$ \\
\hline
\end{tabular}

\subsubsection{Root Node Cuts}

This experiment determined whether it is generally better to include or exclude root node cuts. The contending methods are (i) root node cuts, CUTS, and (ii) no cuts, NOCUTS .

The 116 MIP instances used for this experiment are shown in Table A.2. In this experiment, the values of the GLPK parameters not involving root node cuts are the same values as those used in the experiment described in Section 6.5.1.

Table 6.3 shows the performance of both contenders. 65 MIP instances were solved by at least 1 contending method. Using root node cuts is clearly better than not using cuts and therefore root node cuts are used in the contending node selection methods experiments. The complete results of using default GLPK with root node cuts are shown in Appendix B.2. 


\subsubsection{Node Selection Parameters}

The experiment that determined the best node selection configuration to use when solving MIPs is described in Chapter 7, as well as the contending node selection configurations used in this experiment. There are state of the art contending node selection methods that are not available in GLPK, so these methods were implemented and added to GLPK for this experiment. These methods are listed below.

- The best-estimate backtracking node selection method (Section 3.2.2).

- The best-projection aspiration backtrack triggering method (Sections 3.2.1 and $3.4)$.

- The best-estimate pseudo-cost aspiration backtrack triggering method (Sections 3.2.2 and 3.4).

- The hybrid best-estimate and best-first backtracking node selection method (Section 3.2.2).

- The method that triggers backtracking after every node is solved. 


\section{Chapter 7}

\section{Experimental Results}

The purpose of the experiments in this section is to test all configurations of backtracking node selection, backtrack triggering, and active node search threshold methods to determine which is the best configuration to use for solving MIP instances. All configurations are tested to assess how well each option for each component of a configuration performs in conjunction with various options for the other components.

The options for backtracking node selection method, backtrack triggering method and active node search threshold are described below.

- Backtracking node selection methods:

- State of the art methods that are available in GLPK.

DEPF: Depth-first (Section 3.1).

BREF: Breadth-first (Section 3.1).

DEBP: Default best-projection (Default GLPK, Section 3.2.1).

BESF: Best-first (Section 3.1).

- State of the art methods that were added to GLPK for this experiment.

BEES: Best-estimate (Section 3.2.2).

BFBE: BEES interleaved with BESF (Section 3.2.2).

- New methods that are proposed in this thesis. 
DIST: Distribution (Method proposed in Section 5.2).

MOBP: Modified best-projection (Method proposed in Section 5.1).

- Backtrack triggering methods:

- State of the art methods that are available in GLPK.

NONA: Non-aspiration backtacking: trigger backtracking only at leaf nodes (Default GLPK).

- State of the art methods that were added to GLPK for this experiment.

ALLT: Perform backtracking node selection after every node solution.

DBPA: Default best-projection aspiration (Section 3.4).

PCAS: Pseudo-cost (best-estimate) aspiration (Section 3.4).

- New methods that are proposed in this thesis.

LEXA: Linear feasibility depth extrapolation aspiration (Proposed in Section 5.3).

MPAS: Modified best-projection aspiration (Proposed in Section 5.1).

- Active node search threshold (Proposed in Section 5.4) options:

NOAN: Do not use ANST (Default GLPK).

ANST: Use ANST.

Each state of the art method that is not listed above is excluded from this experiment because (i) there are no documented empirical studies in which the excluded method outperforms all of the above methods, (ii) the principal behavior of the excluded method relies on user defined parameters, or (iii) the details required to properly implement the excluded method are not documented.

One example of a node selection configuration is DEBP-NONA-NOAN. In this configuration best-projection backtracking node selection is used whenever a leaf node is reached, and the ANST method is not used. This is the default configuration in 
GLPK. All of the competitor configurations in this experiment are listed in Table 7.1. This list excludes any configuration in which depth-first backtracking is combined with any backtrack triggering method other than NONA because depth-first backtracking will choose a child of the most recently solved node if the most recently solved node is not a leaf. Also excluded from this list is any configuration in which ANST is combined with either depth-first or breadth-first node selection since depth-first is the simple node selection method used in ANST and since breadth-first is usually no more costly than depth-first.

The list of competitor configurations is quite large; there are 79 configurations. Due to the impractical amount of time required to evaluate the performance of every configuration on a sufficiently large set of MIP instances, this experiment is carried out in two stages. The purpose of the first stage is to reduce the list of competitor configurations to a small subset of the most promising configurations. The set of MIP instances used in stage 1 is composed such that the performance of all of the competitor configurations can be evaluated within a reasonable amount of time, i.e. these are the models that tend to get solved within a shorter timeframe. The details and results of stage 1 of this experiment are presented in Section 7.1. The purpose of the second stage is to evaluate the performance of the most promising configurations determined in stage 1 on a larger set of MIP instances. The details and results of stage 2 of this experiment are presented in Section 7.2.

For both stages of this experiment, if the amount of memory used when solving a MIP instance exceeds 1.5 gigabytes then the depth-first backtracking node selection method without an aspiration backtrack triggering method is used until the memory usage drops below 1 gigabytes. This method is commonly used to avoid using the extremely slow swap space of memory [4]. This threshold was never exceeded in these experiments. 
Table 7.1: List of all competitor node selection configurations. The configurations that are available in the original GLPK solver are in boldface.

\begin{tabular}{|l|l|l|}
\hline BEES-ALLT-ANST & BFBE-DBPA-NOAN & DEPF-NONA-NOAN \\
BEES-ALLT-NOAN & BFBE-LEXA-ANST & DIST-ALLT-ANST \\
BEES-DBPA-ANST & BFBE-LEXA-NOAN & DIST-ALLT-NOAN \\
BEES-DBPA-NOAN & BFBE-MPAS-ANST & DIST-DBPA-ANST \\
BEES-LEXA-ANST & BFBE-MPAS-NOAN & DIST-DBPA-NOAN \\
BEES-LEXA-NOAN & BFBE-NONA-ANST & DIST-LEXA-ANST \\
BEES-MPAS-ANST & BFBE-NONA-NOAN & DIST-LEXA-NOAN \\
BEES-MPAS-NOAN & BFBE-PCAS-ANST & DIST-MPAS-ANST \\
BEES-NONA-ANST & BFBE-PCAS-NOAN & DIST-MPAS-NOAN \\
BEES-NONA-NOAN & BREF-ALLT-NOAN & DIST-NONA-ANST \\
BEES-PCAS-ANST & BREF-DBPA-NOAN & DIST-NONA-NOAN \\
BEES-PCAS-NOAN & BREF-LEXA-NOAN & DIST-PCAS-ANST \\
BESF-ALLT-ANST & BREF-MPAS-NOAN & DIST-PCAS-NOAN \\
BESF-ALLT-NOAN & BREF-NONA-NOAN & MOBP-ALLT-ANST \\
BESF-DBPA-ANST & BREF-PCAS-NOAN & MOBP-ALLT-NOAN \\
BESF-DBPA-NOAN & DEBP-ALLT-ANST & MOBP-DBPA-ANST \\
BESF-LEXA-ANST & DEBP-ALLT-NOAN & MOBP-DBPA-NOAN \\
BESF-LEXA-NOAN & DEBP-DBPA-ANST & MOBP-LEXA-ANST \\
BESF-MPAS-ANST & DEBP-DBPA-NOAN & MOBP-LEXA-NOAN \\
BESF-MPAS-NOAN & DEBP-LEXA-ANST & MOBP-MPAS-ANST \\
BESF-NONA-ANST & DEBP-LEXA-NOAN & MOBP-MPAS-NOAN \\
BESF-NONA-NOAN & DEBP-MPAS-ANST & MOBP-NONA-ANST \\
BESF-PCAS-ANST & DEBP-MPAS-NOAN & MOBP-NONA-NOAN \\
BESF-PCAS-NOAN & DEBP-NONA-ANST & MOBP-PCAS-ANST \\
BFBE-ALLT-ANST & DEBP-NONA-NOAN & MOBP-PCAS-NOAN \\
BFBE-ALLT-NOAN & DEBP-PCAS-ANST & \\
BFBE-DBPA-ANST & DEBP-PCAS-NOAN & \\
\hline
\end{tabular}




\subsection{Identification of Promising Configurations}

The purpose of this experiment is to test all configurations of backtracking node selection, backtrack triggering, and active node search threshold methods on a small set of MIP instances in order to determine which are the most promising configurations to test on a larger set of MIP instances.

The time limit for this experiment is 30 minutes per MIP solution. The MIP instances used in this experiment are those in Table A.1 that were solved within 30 minutes using the default GLPK node selection configuration, DEBP-NONA-NOAN. There are 79 of these MIP instances and they are shown in Table A.3. Choosing the set of MIP instances in this manner biases the results to favor DEBP-NONANOAN by removing any MIP instances that are not solved within the time limit using DEBP-NONA-NOAN even though these MIP instances may be solved within the time limit using a different configuration. This experiment required nearly 3 weeks of computation time to complete.

In order to determine how each competitor configuration ranks compared to the others, a method is needed that incorporates the three performance measures, TOTTM, MRATE and FAIL, described in Section 6.3 into a single measure. Towards this end, the following definitions are used to rank each competitor configuration.

TR: Time rank is the rank of a competitor configuration according to TOTTM.

$R R$ : Ratio rank is the rank of a competitor configuration according to MRATE.

$S R$ : Sum rank is the measure used to determine the overall rank of each competitor configuration. SR is computed using Equation 7.1. Configurations with smaller values of $\mathrm{SR}$ are better.

$R$ : The rank of each competitor configuration according to SR.

$$
S R=F A I L+T R+R R
$$


Equation 7.1 is used to rank the competitor configurations because it is desirable for a competitor configuration to have the smallest value of $F A I L, T R$, and $R R$. Addition is used to prevent one of these measures from dominating the others. Table 7.5 shows that the largest difference either between $T R$ and $R$, or between $R R$ and $R$, is 11 for BFBE-PCAS-NOAN which has $R=43, T R=52$, and $R R=32$. The average of this difference over all configurations is 3.2. FAIL is added to $S R$ instead of the ranking according to $F A I L$ because (i) $F A I L$ is integer valued, and (ii) by adding the $F A I L$ rank to $S R$, the difference between the values of $F A I L$ for two configurations is lost if this difference is greater than the difference in their ranks. In this experiment, the ranking of each configuration according to $F A I L$ is equal to $F A I L+1$ for all but the worst 3 configurations, therefore either method of computing $S R$ would give similar results.

Table 7.2 shows $M R A T E$ and $R R$ as well as the variance $M V A R$ in $r_{M I}$ (see Page 67 for definition) for each configuration. $R R$ is 19 for the best state of the art configuration, and its value of $M R A T E$ that is $16 \%$ worse than that of the smallest value of MRATE. This table shows that the range in $M V A R$ is quite large, the smallest value being 0.52 and the largest being 250679 , and that the smaller values of $M V A R$ tend to correspond to smaller values of MRATE. Note that the smallest $M V A R$ corresponds to the smallest $M R A T E$, and that both of these are associated with DIST-ALLT-NOAN, indicating that this is a very high quality method. Figure 7.1 shows a plot of MRATE, and the 10th and 90th percentiles for $r_{M I}$ for each configuration. This plot also shows that smaller values of $M R A T E$ tend to correspond to smaller values of both percentiles, especially the 90th percentile.

Table 7.2: Performance of each configuration in Table 7.1 with respect to MRATE. Also shown is the variance of $r_{M I}$ over all MIP instances for each configuration. The configurations that are available in the original GLPK solver are in boldface.

\begin{tabular}{|c|c|c|c|}
\hline RR & Configuration & MRATE & MVAR \\
\hline 1 & DIST-ALLT-NOAN & 1.28927 & 0.52 \\
\hline \multicolumn{4}{|c|}{ Continued on next page } \\
\hline
\end{tabular}


Table 7.2 - continued from previous page

\begin{tabular}{|c|c|c|c|}
\hline RR & Configuration & MRATE & MVAR \\
\hline 2 & MOBP-MPAS-ANST & 1.35222 & 50.37 \\
3 & MOBP-ALLT-NOAN & 1.35982 & 24.63 \\
4 & MOBP-MPAS-NOAN & 1.36108 & 50.88 \\
5 & MOBP-ALLT-ANST & 1.36519 & 32.72 \\
6 & MOBP-PCAS-ANST & 1.37487 & 50.21 \\
7 & MOBP-LEXA-ANST & 1.38146 & 61.11 \\
8 & MOBP-PCAS-NOAN & 1.38447 & 49.42 \\
9 & MOBP-DBPA-ANST & 1.38864 & 63.11 \\
10 & DIST-MPAS-ANST & 1.39187 & 113.8 \\
11 & MOBP-LEXA-NOAN & 1.41064 & 60.94 \\
12 & MOBP-DBPA-NOAN & 1.41183 & 62.67 \\
13 & MOBP-NONA-ANST & 1.41603 & 64.79 \\
14 & MOBP-NONA-NOAN & 1.42075 & 62.46 \\
15 & BFBE-ALLT-ANST & 1.43168 & 0.97 \\
16 & BFBE-MPAS-ANST & 1.45506 & 99.28 \\
17 & DIST-MPAS-NOAN & 1.46461 & 105.76 \\
18 & DIST-DBPA-NOAN & 1.47475 & 69.85 \\
19 & BFBE-ALLT-NOAN & 1.53420 & 1.53 \\
20 & BFBE-PCAS-ANST & 1.55149 & 108.2 \\
21 & DIST-DBPA-ANST & 1.55960 & 78.2 \\
22 & DIST-LEXA-NOAN & 1.56728 & 76.09 \\
23 & BFBE-DBPA-ANST & 1.56836 & 23.77 \\
24 & BFBE-LEXA-ANST & 1.59054 & 102.7 \\
25 & DIST-ALLT-ANST & 1.59373 & 170.65 \\
26 & DIST-PCAS-NOAN & 1.60639 & 195.85 \\
27 & DIST-NONA-ANST & 1.61207 & 74.7 \\
28 & BFBE-NONA-ANST & 1.61824 & 66.24 \\
29 & BEES-PCAS-ANST & 1.62567 & 112.87 \\
30 & DEBP-DBPA-NOAN & 1.63204 & 85.86 \\
31 & DIST-PCAS-ANST & 1.64611 & 201.61 \\
32 & BEES-MPAS-ANST & 1.64937 & 117.82 \\
33 & DEBP-DBPA-ANST & 1.65889 & 75 \\
34 & DEBP-PCAS-ANST & 1.67976 & 41.47 \\
35 & BFBE-MPAS-NOAN & 1.68251 & 380.18 \\
36 & DEBP-ALLT-ANST & 1.68649 & 13.53 \\
37 & BFBE-LEXA-NOAN & 1.69066 & 313.12 \\
38 & DEBP-LEXA-NOAN & 1.70648 & 90.84 \\
39 & DIST-NONA-NOAN & 1.70819 & 76.69 \\
40 & DIST-LEXA-ANST & 1.71448 & 99.53 \\
41 & BFBE-NONA-NOAN & 1.72498 & 169.42 \\
42 & DEBP-NONA-NOAN & 1.73127 & 92.05 \\
43 & BFBE-PCAS-NOAN & 1.73483 & 910 \\
44 & BEES-DBPA-ANST & 1.74104 & 116.75 \\
45 & BEES-LEXA-ANST & 1.75071 & 116.44 \\
46 & DEBP-NONA-ANST & 1.75084 & 75.54 \\
\hline & & & \\
& & & \\
& &
\end{tabular}


Table 7.2 - continued from previous page

\begin{tabular}{|c|c|c|c|}
\hline RR & Configuration & MRATE & MVAR \\
\hline 47 & BFBE-DBPA-NOAN & 1.75157 & 190.93 \\
48 & BEES-ALLT-ANST & 1.75423 & 155.33 \\
49 & DEBP-PCAS-NOAN & 1.75796 & 156 \\
50 & BEES-NONA-ANST & 1.76326 & 115.47 \\
51 & DEBP-LEXA-ANST & 1.76442 & 117.23 \\
52 & BEES-PCAS-NOAN & 1.76983 & 112.92 \\
53 & BEES-MPAS-NOAN & 1.77120 & 115.09 \\
54 & BESF-PCAS-ANST & 1.88164 & 86.18 \\
55 & DEBP-MPAS-NOAN & 1.89579 & 215.62 \\
56 & BESF-LEXA-ANST & 1.89786 & 79.79 \\
57 & DEBP-MPAS-ANST & 1.89901 & 387.24 \\
58 & DEBP-ALLT-NOAN & 1.91466 & 2609.09 \\
59 & BEES-DBPA-NOAN & 1.91629 & 322.56 \\
60 & BEES-LEXA-NOAN & 1.91848 & 315.19 \\
61 & BESF-LEXA-NOAN & 1.92596 & 170.11 \\
62 & BEES-ALLT-NOAN & 1.92709 & 328.18 \\
63 & BESF-DBPA-ANST & 1.95607 & 193.56 \\
64 & BESF-NONA-NOAN & 1.96045 & 170.92 \\
65 & BESF-PCAS-NOAN & 1.96116 & 162.79 \\
66 & BESF-NONA-ANST & 1.96341 & 85.02 \\
67 & BESF-DBPA-NOAN & 1.96601 & 186.24 \\
68 & BESF-MPAS-ANST & 1.99262 & 370.14 \\
69 & BESF-MPAS-NOAN & 2.04399 & 165.5 \\
70 & BEES-NONA-NOAN & 2.06815 & 354.12 \\
71 & BREF-LEXA-NOAN & 2.07558 & 551.76 \\
72 & BREF-DBPA-NOAN & 2.09071 & 566.65 \\
73 & BREF-NONA-NOAN & 2.10032 & 566.16 \\
74 & BESF-ALLT-ANST & 2.18260 & 310.81 \\
75 & BESF-ALLT-NOAN & 2.33030 & 557.86 \\
76 & BREF-PCAS-NOAN & 2.39173 & 540.84 \\
77 & DEPF-NONA-NOAN & 2.71091 & 250679.22 \\
78 & BREF-MPAS-NOAN & 3.00261 & 3670.75 \\
79 & BREF-ALLT-NOAN & 4.44186 & 3921.76 \\
\hline \hline
\end{tabular}

Table 7.4 shows the ranking of each configuration according to TOTTM. TR is 21 for the best state of the art configuration, and its value of TOTTM is $11.7 \%$ worse than that of the smallest value of TOTTM.

Table 7.5 shows the performance of each competitor configuration along with their ranks. Standard node selection methods such as depth-first (DEPF-NONA-NOAN), best-first (BESF-NONA-NOAN), and breadth-first (BREF-NONA-NOAN) ranked 


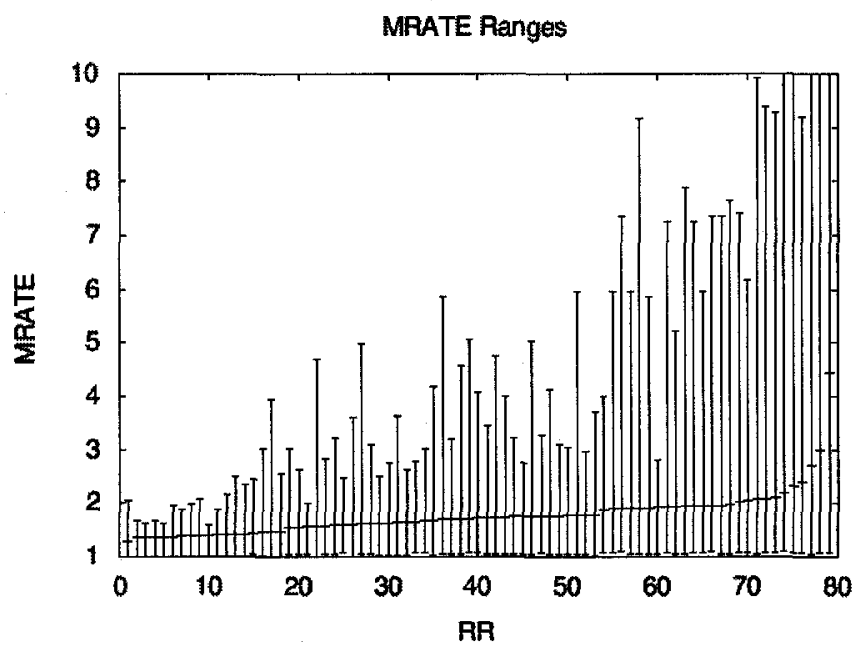

Figure 7.1: A plot showing MRATE, $r_{p 10}$, and $r_{p 90}$ for each configuration. $r_{p 10}$ and $r_{p 90}$ are the 10th and 90th percentiles, respectively, of $r_{M I}$ (see Page 67 for definition) over all MIP instances for a given configuration. Each $\mathrm{x}$-axis value of $R R$ uniquely corresponds to a configuration as is shown in Table 7.2.

76, 64, and 71 respectively. The value of FAIL for DEBP-NONA-NOAN is 0 because the set of MIP instances used in this experiment excludes any MIP instance that was not solved by DEBP-NONA-NOAN within the time limit. The top 21 ranked competitor configurations involve at least one method out of those newly proposed in Sections 5.1 through 5.4. The top ranked competitor configuration, MOBP-MPASANST, consists only of methods proposed in Sections 5.1 and 5.4. Each of the new methods proposed in Sections 5.1 through 5.4 are used at least once in the top 7 ranked competitor configurations. For this reason, these top 7 ranked configurations will be tested in the final node selection experiment described in Section 7.2. The best state of the art configuration, DEBP-DBPA-NOAN, is ranked 22 whereas the default GLPK configuration, DEBP-NONA-NOAN, is ranked 39. These two configurations are also tested in the final node selection experiment to provide a comparison to the best state of the art and to the baseline GLPK.

Analysis of variance (ANOVA) tests on $r_{M I}$ show that there is statistical significance in the differences between the best ranked state of the art configuration 
Table 7.3: The p-values of the analysis of variance (ANOVA) tests of $M R A T E$ between the best ranked state of the art configuration DEBP-DBPA-NOAN and each of the top seven ranked configurations. Smaller p-values mean that the difference between the values of $M R A T E$ for considered methods is more significant.

\begin{tabular}{|l|c|}
\hline DEBP-DBPA-NOAN vs. & p-value \\
\hline DIST-ALLT-NOAN & 0.05 \\
DIST-MPAS-ANST & 0.11 \\
MOBP-LEXA-ANST & 0.1 \\
MOBP-PCAS-NOAN & 0.12 \\
MOBP-PCAS-ANST & 0.1 \\
MOBP-MPAS-NOAN & 0.06 \\
MOBP-MPAS-ANST & 0.05 \\
\hline
\end{tabular}

DEBP-DBPA-NOAN and that of each of the top 7 ranked configurations with respect to MRATE. The p-values of these tests are shown in Table 7.3. ANOVA tests also show that there is very little statistical significance in the differences among the top 7 methods with respect to MRATE.

To determine the overall effect of using ANST versus not using it, the arithmetic means of each of the performance measures ( $\overline{T O T T M}, \overline{M R A T E}$ and $\overline{F A I L})$ are computed over all configurations with ANST and compared to the corresponding means taken over all configurations with NOAN. All configurations with BREF and DEPF are excluded from the means since neither of these methods is combined with ANST in this experiment. Table 7.6 shows that on average, ANST outperforms NOAN for all three of these measures. With respect to $\overline{F A I L}$, ANST improves performance by about $22.5 \%$. With respect to $\overline{T O T T M}$, ANST improves performance by about $5.1 \%$. With respect to $\overline{M R A T E}$, ANST improves performance by about $1.7 \%$.

Table 7.7 shows the values of $\overline{T O T T M}, \overline{M R A T E}$ and $\overline{F A I L}$ over all of the configurations, separating them according to backtrack triggering method. Figures 7.2, 7.3 , and 7.4 show that the performance with respect to solving MIPs quickly of any 
Table 7.4: Performance of each configuration in Table 7.1 with respect to TOTTM. The configurations that are available in the original GLPK solver are in boldface.

\begin{tabular}{|c|c|c|c|c|c|}
\hline TR & Configuration & TOTTM & TR & Configuration & TOTTM \\
\hline 1 & MOBP-PCAS-ANST & 16812.65 & 41 & BFBE-MPAS-NOAN & 21122.74 \\
\hline 2 & MOBP-PCAS-NOAN & 16861.29 & 42 & BEES-PCAS-ANST & 21775.88 \\
\hline 3 & MOBP-MPAS-ANST & 17115.24 & 43 & BFBE-NONA-NOAN & 21827.77 \\
\hline 4 & MOBP-MPAS-NOAN & 17393.7 & 44 & BFBE-ALLT-NOAN & 22000.64 \\
\hline 5 & BFBE-MPAS-ANST & 17669.74 & 45 & BEES-ALLT-ANST & 22031.86 \\
\hline 6 & DIST-MPAS-ANST & 17695.79 & 46 & BEES-MPAS-ANST & 22169.74 \\
\hline 7 & DIST-ALLT-NOAN & 17776.13 & 47 & BFBE-DBPA-NOAN & 22257.84 \\
\hline 8 & MOBP-LEXA-ANST & 17906.25 & 48 & BEES-DBPA-ANST & 22365.05 \\
\hline 9 & MOBP-NONA-NOAN & 17970.21 & 49 & BEES-LEXA-ANST & 22514.53 \\
\hline 10 & MOBP-ALLT-ANST & 18045.45 & 50 & BEES-NONA-ANST & 22520.3 \\
\hline 11 & MOBP-DBPA-ANST & 18075.98 & 51 & DEBP-MPAS-ANST & 22643.89 \\
\hline 12 & MOBP-DBPA-NOAN & 18143.23 & 52 & BFBE-PCAS-NOAN & 22976.51 \\
\hline 13 & MOBP-LEXA-NOAN & 18183.25 & 53 & DEBP-ALLT-NOAN & 23087.6 \\
\hline 14 & MOBP-ALLT-NOAN & 18201.74 & 54 & DEBP-MPAS-NOAN & 23710.79 \\
\hline 15 & MOBP-NONA-ANST & 18279.89 & 55 & BESF-PCAS-ANST & 23855.92 \\
\hline 16 & DIST-NONA-ANST & 18290.62 & 56 & BESF-LEXA-ANST & 24022.76 \\
\hline 17 & DIST-DBPA-ANST & 18455.03 & 57 & BESF-LEXA-NOAN & 24096.97 \\
\hline 18 & DIST-MPAS-NOAN & 18577.81 & 58 & BESF-NONA-ANST & 24156.89 \\
\hline 19 & BFBE-LEXA-ANST & 18621.14 & 59 & BEES-MPAS-NOAN & 24303.55 \\
\hline 20 & DIST-DBPA-NOAN & 18986.03 & 60 & BESF-DBPA-ANST & 24345.53 \\
\hline 21 & DEBP-DBPA-NOAN & 19042.51 & 61 & BEES-LEXA-NOAN & 24393.86 \\
\hline 22 & DIST-LEXA-NOAN & 19091.69 & 62 & BESF-NONA-NOAN & 24472.94 \\
\hline 23 & DIST-LEXA-ANST & 19439 & 63 & BEES-DBPA-NOAN & 24534.95 \\
\hline 24 & DEBP-DBPA-ANST & 19455.25 & 64 & BESF-PCAS-NOAN & 24559.31 \\
\hline 25 & BFBE-DBPA-ANST & 19548.95 & 65 & BESF-DBPA-NOAN & 24595.95 \\
\hline 26 & DEBP-ALLT-ANST & 19553.35 & 66 & BEES-PCAS-NOAN & 24649.17 \\
\hline 27 & BFBE-NONA-ANST & 19619.03 & 67 & BESF-MPAS-ANST & 24657.48 \\
\hline 28 & DIST-NONA-NOAN & 19869.09 & 68 & BEES-ALLT-NOAN & 25029.99 \\
\hline 29 & DEBP-NONA-ANST & 19870.05 & 69 & BEES-NONA-NOAN & 25343.76 \\
\hline 30 & DIST-ALLT-ANST & 19963.48 & 70 & BESF-MPAS-NOAN & 25414.45 \\
\hline 31 & BFBE-ALLT-ANST & 19967.51 & 71 & BREF-NONA-NOAN & 25816.53 \\
\hline 32 & BFBE-PCAS-ANST & 19992.11 & 72 & BREF-LEXA-NOAN & 25855.29 \\
\hline 33 & DIST-PCAS-ANST & 20190.27 & 73 & BREF-DBPA-NOAN & 26079.39 \\
\hline 34 & DEBP-LEXA-NOAN & 20240.77 & 74 & BESF-ALLT-ANST & 27829 \\
\hline 35 & DEBP-PCAS-ANST & 20390.92 & 75 & BESF-ALLT-NOAN & 28713.73 \\
\hline 36 & DEBP-NONA-NOAN & 20584.63 & 76 & DEPF-NONA-NOAN & 30570.64 \\
\hline 37 & DIST-PCAS-NOAN & 20650.23 & 77 & BREF-PCAS-NOAN & 32525.43 \\
\hline 38 & DEBP-LEXA-ANST & 20832.68 & 78 & BREF-MPAS-NOAN & 36409.78 \\
\hline 39 & DEBP-PCAS-NOAN & 20900.44 & 79 & BREF-ALLT-NOAN & 45973.67 \\
\hline 40 & BFBE-LEXA-NOAN & 21077.97 & & & \\
\hline
\end{tabular}


Table 7.5: Performance of each configuration in Table 7.1 and their rankings. The configurations that are available in the original GLPK solver are in boldface. The smallest values of $F A I L, T R$, and $R R$ are also in boldface.

\begin{tabular}{|c|c|c|c|c|c|c|c|c|c|}
\hline$\overline{\mathbf{R}}$ & Configuration & FAIL & TR & $\overline{\mathrm{RR}}$ & $\mathrm{R}$ & Configuration & FAIL & TR & $\overline{\mathrm{RR}}$ \\
\hline 1 & MOBP-MPAS-ANST & 1 & 3 & 2 & $\overline{41}$ & DEBP-PCAS-ANST & 3 & 35 & 48 \\
\hline 2 & MOBP-MPAS-NOAN & 1 & 4 & 3 & 42 & DEBP-LEXA-ANST & 3 & 38 & 46 \\
\hline 3 & MOBP-PCAS-ANST & 1 & 1 & 7 & 43 & BFBE-PCAS-NOAN & 5 & 52 & 32 \\
\hline 4 & DIST-ALLT-NOAN & 2 & 7 & 1 & 44 & BFBE-DBPA-NOAN & 3 & 47 & 40 \\
\hline 5 & DIST-MPAS-ANST & 1 & 6 & 4 & 45 & BEES-ALLT-ANST & 2 & 45 & 45 \\
\hline 5 & MOBP-PCAS-NOAN & 1 & 2 & 8 & 45 & BEES-MPAS-ANST & 3 & 46 & 43 \\
\hline 7 & MOBP-LEXA-ANST & 2 & 8 & 6 & 45 & BEES-PCAS-ANST & 3 & 42 & 47 \\
\hline 8 & MOBP-DBPA-ANST & 2 & 11 & 5 & 45 & DEBP-PCAS-NOAN & 3 & 39 & 50 \\
\hline 9 & BFBE-MPAS-ANST & 1 & 5 & 16 & 49 & BEES-DBPA-ANST & 3 & 48 & 42 \\
\hline 10 & MOBP-DBPA-NOAN & 2 & 12 & 9 & 50 & BEES-NONA-ANST & 3 & 50 & 49 \\
\hline 10 & MOBP-NONA-NOAN & 2 & 9 & 12 & 51 & BEES-LEXA-ANST & 4 & 49 & 51 \\
\hline 12 & MOBP-LEXA-NOAN & 2 & 13 & 10 & 52 & DEBP-MPAS-ANST & 3 & 51 & 52 \\
\hline 13 & MOBP-ALLT-ANST & 2 & 10 & 14 & 53 & DEBP-ALLT-NOAN & 4 & 53 & 53 \\
\hline 14 & MOBP-NONA-ANST & 2 & 15 & 13 & 54 & DEBP-MPAS-NOAN & 3 & 54 & 55 \\
\hline 15 & DIST-MPAS-NOAN & 2 & 18 & 11 & 55 & BESF-PCAS-ANST & 5 & 55 & 57 \\
\hline 15 & MOBP-ALLT-NOAN & 2 & 14 & 15 & 56 & BESF-LEXA-NOAN & 6 & 57 & 56 \\
\hline 17 & BFBE-LEXA-ANST & 1 & 19 & 19 & 57 & BESF-LEXA-ANST & 5 & 56 & 59 \\
\hline 17 & DIST-DBPA-NOAN & 2 & 20 & 17 & 58 & BEES-DBPA-NOAN & 5 & 63 & 54 \\
\hline 17 & DIST-NONA-ANST & 1 & 16 & 22 & 59 & BEES-MPAS-NOAN & 5 & 59 & 61 \\
\hline 20 & DIST-DBPA-ANST & 1 & 17 & 26 & 60 & BESF-DBPA-ANST & 6 & 60 & 60 \\
\hline 21 & DIST-LEXA-NOAN & 2 & 22 & 21 & 60 & BESF-NONA-ANST & 6 & 58 & 62 \\
\hline 22 & DEBP-DBPA-NOAN & $\mathbf{0}$ & 21 & 27 & 62 & BESF-DBPA-NOAN & 6 & 65 & 58 \\
\hline 23 & BFBE-ALLT-ANST & 2 & 31 & 18 & 63 & BEES-LEXA-NOAN & 5 & 61 & 66 \\
\hline 24 & BFBE-PCAS-ANST & 2 & 32 & 20 & 64 & BESF-NONA-NOAN & 6 & 62 & 65 \\
\hline 25 & BFBE-DBPA-ANST & 1 & 25 & 30 & 64 & BESF-PCAS-NOAN & 6 & 64 & 63 \\
\hline 25 & BFBE-NONA-ANST & 1 & 27 & 28 & 66 & BESF-MPAS-ANST & 6 & 67 & 64 \\
\hline 27 & DEBP-DBPA-ANST & 1 & 24 & 35 & 67 & BEES-PCAS-NOAN & 4 & 66 & 69 \\
\hline 28 & DIST-LEXA-ANST & 1 & 23 & 37 & 68 & BEES-ALLT-NOAN & 4 & 68 & 68 \\
\hline 29 & DEBP-ALLT-ANST & 2 & 26 & 34 & 69 & BESF-MPAS-NOAN & 6 & 70 & 67 \\
\hline 30 & DIST-NONA-NOAN & 2 & 28 & 33 & 70 & BEES-NONA-NOAN & 5 & 69 & 73 \\
\hline 30 & DIST-PCAS-NOAN & 3 & 37 & 23 & 71 & BREF-LEXA-NOAN & 6 & 72 & 70 \\
\hline 32 & DIST-PCAS-ANST & 2 & 33 & 31 & 71 & BREF-NONA-NOAN & 6 & 71 & 71 \\
\hline 33 & BFBE-LEXA-NOAN & 2 & 40 & 25 & 73 & BREF-DBPA-NOAN & 6 & 73 & 72 \\
\hline 34 & BFBE-MPAS-NOAN & 3 & 41 & 24 & 74 & BESF-ALLT-ANST & 8 & 74 & 74 \\
\hline 34 & DIST-ALLT-ANST & 2 & 30 & 36 & 75 & BESF-ALLT-NOAN & 8 & 75 & 75 \\
\hline 36 & DEBP-NONA-ANST & 1 & 29 & 41 & 76 & DEPF-NONA-NOAN & 7 & 76 & 76 \\
\hline 37 & DEBP-LEXA-NOAN & 3 & 34 & 38 & 77 & BREF-PCAS-NOAN & 10 & 77 & 77 \\
\hline 38 & BFBE-ALLT-NOAN & 3 & 44 & 29 & 78 & BREF-MPAS-NOAN & 13 & 78 & 78 \\
\hline 39 & DEBP-NONA-NOAN & $\mathbf{0}$ & 36 & 44 & 79 & BREF-ALLT-NOAN & 19 & 79 & 79 \\
\hline 40 & BFBE-NONA-NOAN & 2 & 43 & 39 & & & & & \\
\hline
\end{tabular}


Table 7.6: Average performance of configurations with NOAN vs ANST. Configurations with BREF and DEPF are excluded. The best values of FAIL, TOTTM, and $M R A T E$ are in boldface.

\begin{tabular}{|l|l|l|l|}
\hline & $\overline{F A I L}$ & $\overline{T O T T M}$ & $\overline{M R A T E}$ \\
\hline NOAN & 3.33 & 21684.53 & 1.72 \\
ANST & $\mathbf{2 . 5 8}$ & $\mathbf{2 0 5 7 4 . 4 2}$ & $\mathbf{1 . 6 9}$ \\
\hline
\end{tabular}

Table 7.7: Average performance of each backtrack triggering method. DEPF-NONANOAN is excluded. The best values of FAIL, TOTTM, and MRATE are in boldface.

\begin{tabular}{|l|l|l|l|}
\hline Aspiration & $\overline{F A I L}$ & $\overline{T O T T M}$ & $\overline{M R A T E}$ \\
\hline DBPA & 2.92 & $\mathbf{2 1 2 2 1 . 9 8}$ & $\mathbf{1 . 7}$ \\
LEXA & 3.23 & 21252.01 & 1.73 \\
NONA & $\mathbf{2 . 8 5}$ & 21432.44 & 1.76 \\
PCAS & 3.69 & 22010.78 & 1.8 \\
MPAS & 3.69 & 22221.9 & 1.76 \\
ALLT & 4.62 & 23705.7 & 1.94 \\
\hline
\end{tabular}

given backtrack triggering method varies greatly depending on the choice of backtracking node selection method. For example, although MOBP-MPAS-ANST is the best ranked configuration, Table 7.7 shows that, on the average, MPAS is inferior to DBPA. Similarly, MOBP-LEXA-ANST ranks better than the highest ranked configuration with DBPA but LEXA is inferior to DBPA on average. Table 7.8 shows that the LEXA backtrack triggering method outperforms the state of the art backtrack triggering methods when either the BREF or BFBE backtracking node selection method is used. It also shows that the MPAS backtrack triggering method outperforms the state of the art backtrack triggering methods when either the BREF, DIST or MOBP node selection method is used.

Figure 7.5 shows that the average, minimum, and maximum rankings for MOBP and DIST are each less than the respective values for all of the state of the art 


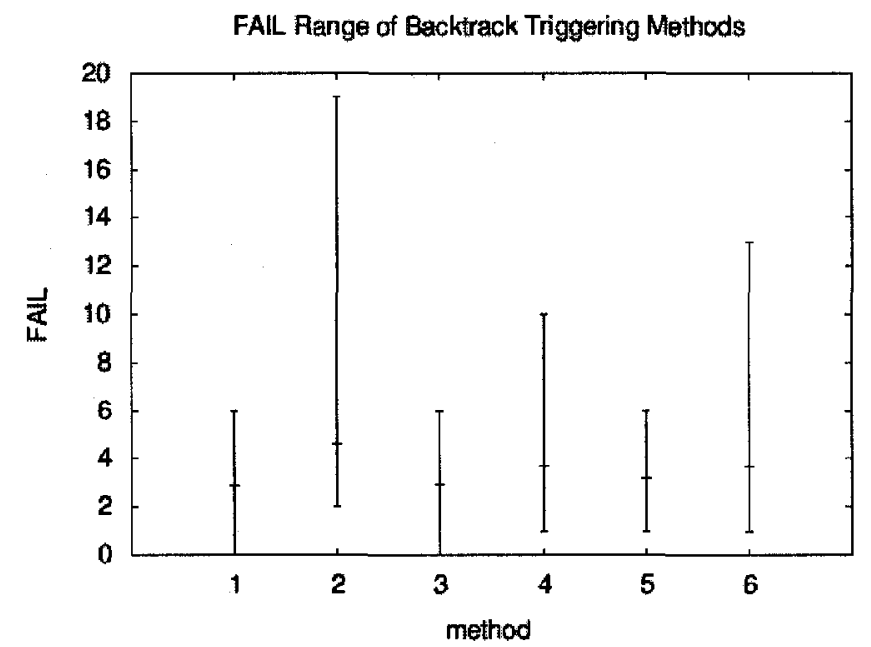

Figure 7.2: A plot showing the average, minimum, and maximum value of $F A I L$ for each of the following backtrack triggering methods: method $1=$ NONA, method 2 $=$ ALLT, method $3=\mathrm{DBPA}$, method $4=$ PCAS, method $5=$ LEXA, method $6=$ MPAS.

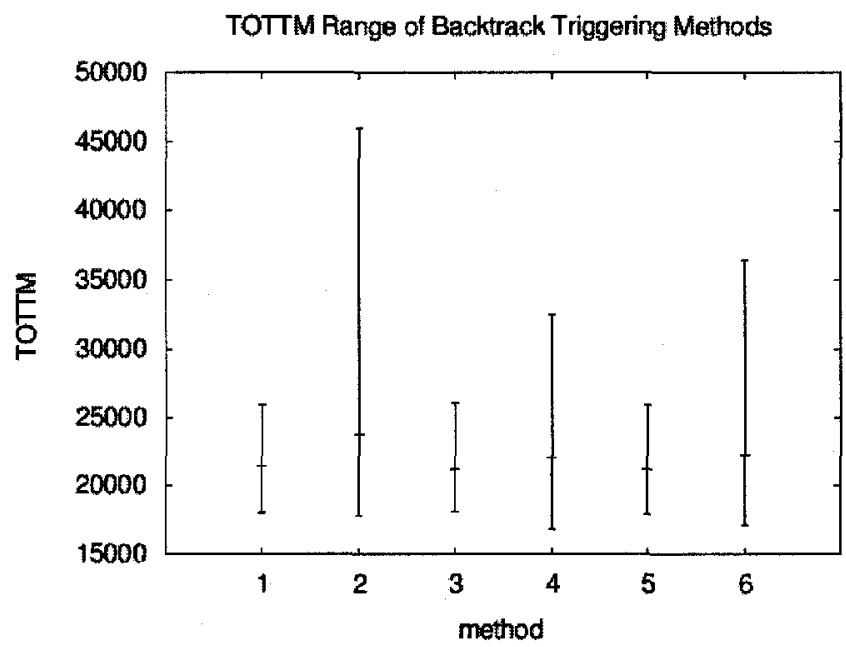

Figure 7.3: A plot showing the average, minimum, and maximum value of TOTTM for each of the following backtrack triggering methods: method $1=$ NONA, method $2=$ ALLT, method $3=$ DBPA, method $4=$ PCAS, method $5=$ LEXA, method $6=$ MPAS. 


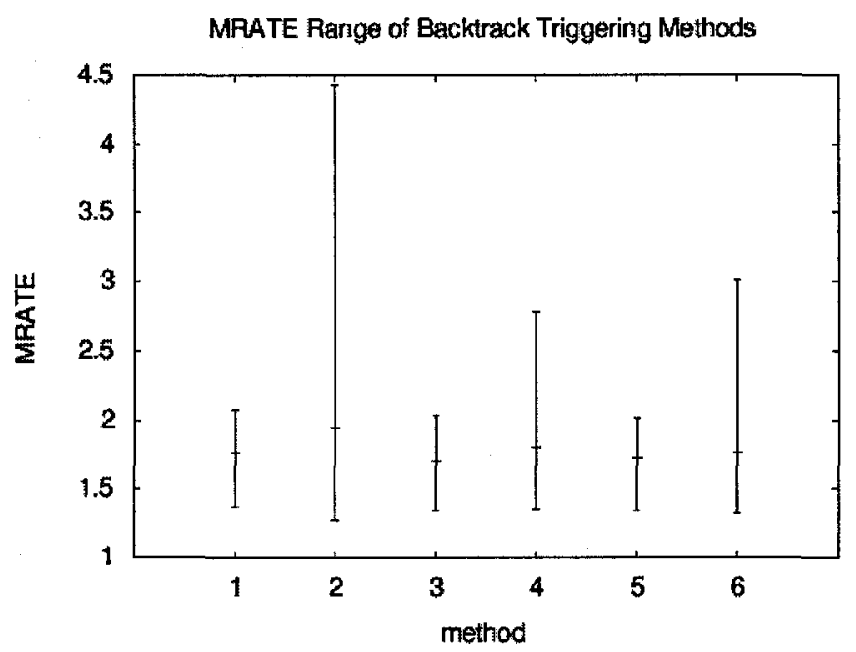

Figure 7.4: A plot showing the average, minimum, and maximum value of $M R A T E$ for each of the following backtrack triggering methods: method $1=$ NONA, method $2=$ ALLT, method $3=$ DBPA, method $4=$ PCAS, method $5=$ LEXA, method $6=$ MPAS.

backtracking node selection methods. Figures 7.6, 7.7, and 7.8 show that the best backtracking node selection method has the smallest range of FAIL, TOTTM, and $M R A T E$. The ranges of FAIL, TOTTM, and MRATE for the backtracking node selection methods tends to be smaller than for backtrack triggering methods.

The complete results of this experiment are given in Appendix B.3. 
Table 7.8: Rank of configurations with the same backtracking node selection option.

\begin{tabular}{|c|c|c|c|c|c|}
\hline $\mathrm{R}$ & Method 1 & $\mathbf{R}$ & Method 2 & $\mathbf{R}$ & Method 3 \\
\hline 1 & MOBP-MPAS-ANST & 4 & DIST-ALLT-NOAN & 9 & BFBE-MPAS-ANST \\
\hline 2 & MOBP-MPAS-NOAN & 5 & DIST-MPAS-ANST & 17 & BFBE-LEXA-ANST \\
\hline 3 & MOBP-PCAS-ANST & 15 & DIST-MPAS-NOAN & 23 & BFBE-ALLT-ANST \\
\hline 5 & MOBP-PCAS-NOAN & 17 & DIST-DBPA-NOAN & 24 & BFBE-PCAS-ANST \\
\hline 7 & MOBP-LEXA-ANST & 17 & DIST-NONA-ANST & 25 & BFBE-DBPA-ANST \\
\hline 8 & MOBP-DBPA-ANST & 20 & DIST-DBPA-ANST & 25 & BFBE-NONA-ANST \\
\hline 10 & MOBP-DBPA-NOAN & 21 & DIST-LEXA-NOAN & 33 & BFBE-LEXA-NOAN \\
\hline 10 & MOBP-NONA-NOAN & 28 & DIST-LEXA-ANST & 34 & BFBE-MPAS-NOAN \\
\hline 12 & MOBP-LEXA-NOAN & 30 & DIST-NONA-NOAN & 38 & BFBE-ALLT-NOAN \\
\hline 13 & MOBP-ALLT-ANST & 30 & DIST-PCAS-NOAN & 40 & BFBE-NONA-NOAN \\
\hline 14 & MOBP-NONA-ANST & 32 & DIST-PCAS-ANST & 43 & BFBE-PCAS-NOAN \\
\hline 15 & MOBP-ALLT-NOAN & 34 & DIST-ALLT-ANST & 44 & BFBE-DBPA-NOAN \\
\hline$\overline{\mathrm{R}}$ & Method 4 & $\mathbf{R}$ & Method 5 & $\mathrm{R}$ & Method 6 \\
\hline 22 & DEBP-DBPA-NOAN & 45 & BEES-ALLT-ANST & 55 & BESF-PCAS-ANST \\
\hline 27 & DEBP-DBPA-ANST & 45 & BEES-MPAS-ANST & 56 & BESF-LEXA-NOAN \\
\hline 29 & DEBP-ALLT-ANST & 45 & BEES-PCAS-ANST & 57 & BESF-LEXA-ANST \\
\hline 36 & DEBP-NONA-ANST & 49 & BEES-DBPA-ANST & 60 & BESF-DBPA-ANST \\
\hline 37 & DEBP-LEXA-NOAN & 50 & BEES-NONA-ANST & 60 & BESF-NONA-ANST \\
\hline 39 & DEBP-NONA-NOAN & 51 & BEES-LEXA-ANST & 62 & BESF-DBPA-NOAN \\
\hline 41 & DEBP-PCAS-ANST & 58 & BEES-DBPA-NOAN & 64 & BESF-NONA-NOAN \\
\hline 42 & DEBP-LEXA-ANST & 59 & BEES-MPAS-NOAN & 64 & BESF-PCAS-NOAN \\
\hline 45 & DEBP-PCAS-NOAN & 63 & BEES-LEXA-NOAN & 66 & BESF-MPAS-ANST \\
\hline 52 & DEBP-MPAS-ANST & 67 & BEES-PCAS-NOAN & 69 & BESF-MPAS-NOAN \\
\hline 53 & DEBP-ALLT-NOAN & 68 & BEES-ALLT-NOAN & 74 & BESF-ALLT-ANST \\
\hline 54 & DEBP-MPAS-NOAN & 70 & BEES-NONA-NOAN & 75 & BESF-ALLT-NOAN \\
\hline $\mathbf{R}$ & Method 7 & & & & \\
\hline$\overline{71}$ & BREF-LEXA-NOAN & & & & \\
\hline 71 & BREF-NONA-NOAN & & & & \\
\hline 73 & BREF-DBPA-NOAN & & & & \\
\hline 77 & BREF-PCAS-NOAN & & & & \\
\hline 78 & BREF-MPAS-NOAN & & & & \\
\hline 79 & BREF-ALLT-NOAN & & & & \\
\hline
\end{tabular}




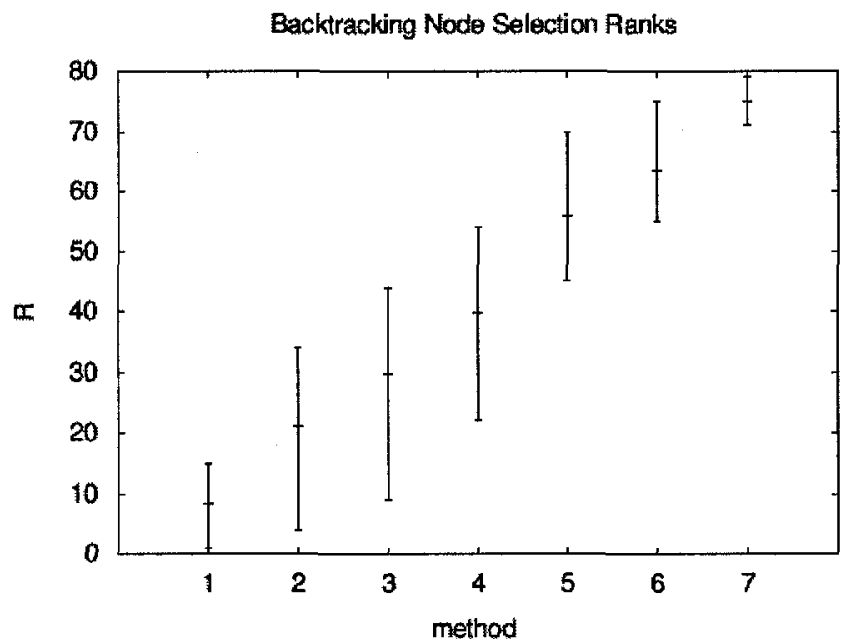

Figure 7.5: A plot showing the average, minimum, and maximum ranking for each of the backtracking node selection methods shown in Table 7.8; method 1: MOBP, method 2: DIST, method 3: BFBE, method 4: DEBP, method 5: BEES, method 6: BESF, method 7: BREF.

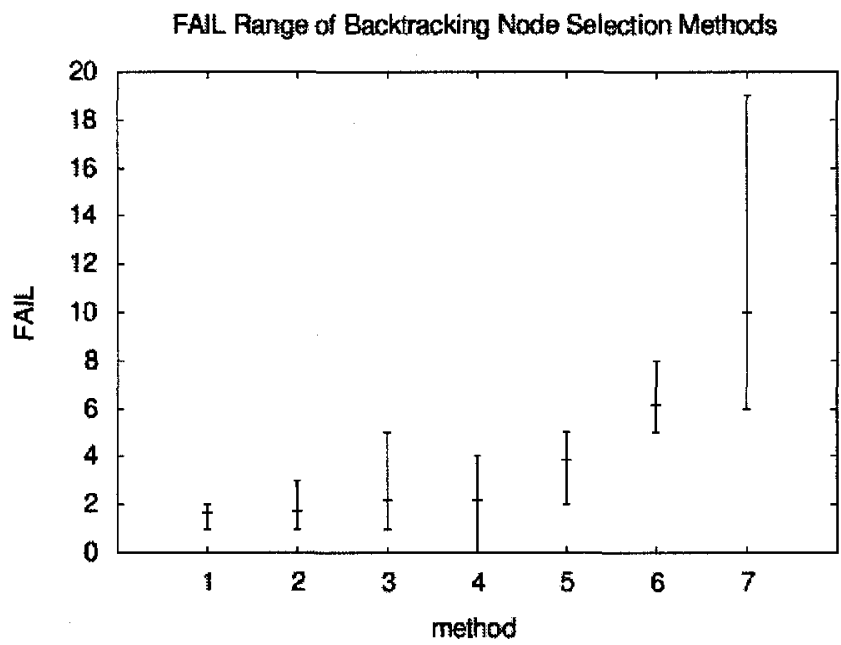

Figure 7.6: A plot showing the average, minimum, and maximum value of $F A I L$ for each of the backtracking node selection methods shown in Table 7.8; method 1: MOBP, method 2: DIST, method 3: BFBE, method 4: DEBP, method 5: BEES, method 6: BESF, method 7: BREF. 


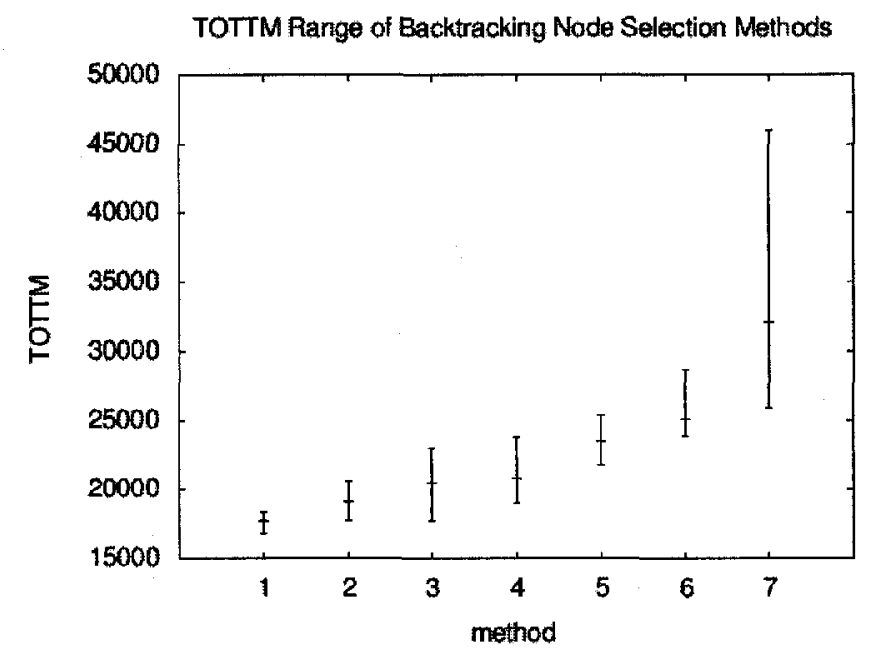

Figure 7.7: A plot showing the average, minimum, and maximum value of TOTTM for each of the backtracking node selection methods shown in Table 7.8; method 1: MOBP, method 2: DIST, method 3: BFBE, method 4: DEBP, method 5: BEES, method 6: BESF, method 7: BREF.

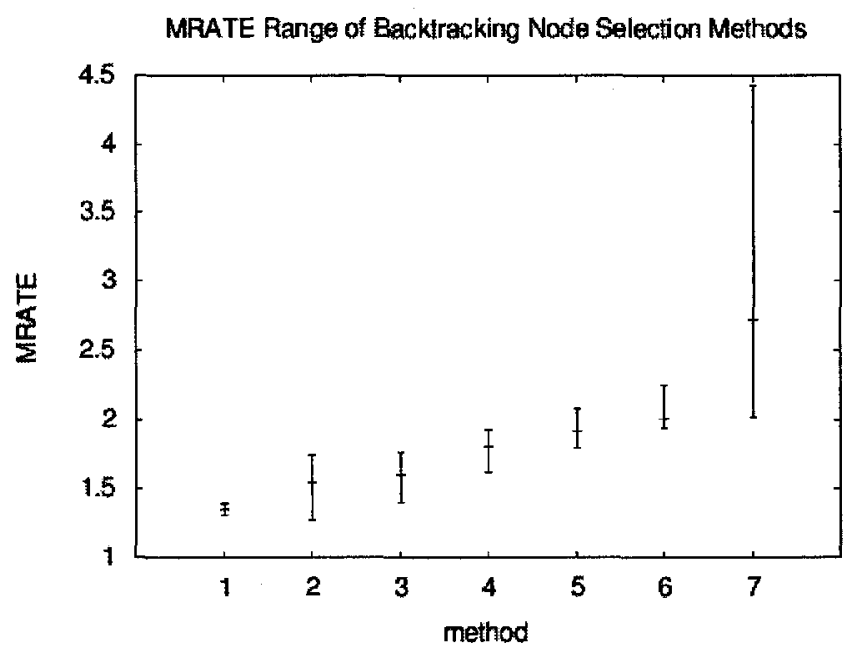

Figure 7.8: A plot showing the average, minimum, and maximum value of $M R A T E$ for each of the backtracking node selection methods shown in Table 7.8; method 1: MOBP, method 2: DIST, method 3: BFBE, method 4: DEBP, method 5: BEES, method 6: BESF, method 7: BREF. 
Table 7.9: List of promising node selection configurations for the stage 2 experiment along with their rank $\mathrm{R}$ determined in Section 7.1 and why they are included in this experiment.

\begin{tabular}{|l|l|l|}
\hline \multicolumn{1}{|c|}{$\mathrm{R}$} & \multicolumn{1}{|c|}{ Configuration } & \multicolumn{1}{c|}{ Description } \\
\hline 39 & DEBP-NONA-NOAN & Default GLPK: included as a baseline for GLPK \\
22 & DEBP-DBPA-NOAN & Top ranked state of the art configuration \\
1 & MOBP-MPAS-ANST & Top ranked proposed configuration \\
2 & MOBP-MPAS-NOAN & Second ranked proposed configuration \\
3 & MOBP-PCAS-ANST & Third ranked proposed configuration \\
4 & DIST-ALLT-NOAN & Fourth ranked proposed configuration \\
5 & DIST-MPAS-ANST & Fifth ranked proposed configuration \\
5 & MOBP-PCAS-NOAN & Fifth ranked proposed configuration \\
7 & MOBP-LEXA-ANST & Highest ranked proposed configuration with LEXA \\
\hline
\end{tabular}

\subsection{Testing of Promising Configurations}

The purpose of this experiment is to perform a more thorough test to determine which of the most promising configurations found in Section 7.1 is best for solving MIPs. Towards this end, both the time limit for this experiment and the number of MIP instances are larger than those used in Section 7.1.

The competitor configurations for this experiment are the top 7 ranked configurations from Section 7.1 as well as the top ranked state of the art and GLPK baseline configurations. Seven of the top ranked configurations are used because each of the new methods proposed in this thesis is used in at least one of these configurations. These configurations are listed in Table 7.9.

The set of MIP instances used in this experiment is listed in Table A.1. This list includes the MIP instances from the experiment in Section 7.1. The time limit for each MIP solution in this experiment is 1 hour. This experiment required over 9 weeks of computation time to complete.

- 272 MIP instances are used in this experiment.

- 109 MIP instances were solved to optimality using at least 1 of the competitor configurations. These instances are listed in Table A.4. 
- 130 MIP instances were not solved to optimality using any configuration, but at least one configuration found a feasible solution within the time limit. These MIP instances are listed in Table A.5.

- 33 MIP instances were not solved and no MIP feasible solution was found using any configuration. These MIP instances are listed in Table A.6.

Table 7.10 shows the performance of each competitor configuration. All but one of the configurations proposed in this thesis have fewer unsolved MIPs than the state of the art configurations. The configurations with the fewest unsolved MIPs are DIST-MPAS-ANST, MOBP-LEXA-ANST, MOBP-PCAS-ANST and MOBP-MPASANST. These configurations all use ANST whereas all of the others do not, which indicates that using ANST increases the chances of solving difficult MIPs within a stated time limit.

Over all MIP instances, TOTTM for the worst proposed configuration, MOBPPCAS-NOAN, is $7.8 \%$ less than the best state of the art configuration, DEBPDBPA-NOAN. In the case of the best proposed configuration, MOBP-MPAS-ANST, TOTTM is $20.7 \%$ less than DEBP-DBPA-NOAN. With respect to MRATE, the worst proposed configuration, MOBP-PCAS-NOAN, is $18.7 \%$ better than the best state of the art configuration, DEBP-DBPA-NOAN. The best proposed configuration, MOBP-MPAS-ANST, has a value of MRATE that is $24.2 \%$ better than DEBP-DBPA-NOAN. The TOTTM for the second best proposed method, DISTMPAS-ANST, is $0.001 \%$ worse than MOBP-MPAS-ANST and the MRATE for DIST-MPAS-ANST is $3.1 \%$ worse than MOBP-MPAS-ANST which indicates that the performance of DIST-MPAS-ANST for solving MIPs is very similar to MOBPMPAS-ANST.

To investigate the effect of any bias introduced by including the results from the first experiment in the second experiment, we recalculated the results with those models removed. The bias is negligible. Over all MIP instances that were not used in the experiment in Section 7.1, TOTTM for the worst proposed configuration, MOBP-PCAS-NOAN, is $6.9 \%$ less than the best state of the art configuration, 
DEBP-DBPA-NOAN. In the case of the best proposed configuration, MOBP-MPASANST, TOTTM is $22 \%$ less than DEBP-DBPA-NOAN. With respect to MRATE, the worst proposed configuration, MOBP-PCAS-NOAN, is $23.5 \%$ better than the best state of the art configuration, DEBP-DBPA-NOAN. The best proposed configuration, MOBP-MPAS-ANST, has a value of MRATE that is $35.2 \%$ better than DEBP-DBPA-NOAN.

ANOVA tests on $r_{M I}$ show that there is statistical significance in the differences between DEBP-DBPA-NOAN and most of the proposed configurations with respect to MRATE. The p-values of these tests are shown in Table 7.11. The MOBP-PCASNOAN configuration is not significantly different from DEBP-DBPA-NOAN, but it is also the worst ranked proposed configuration. 


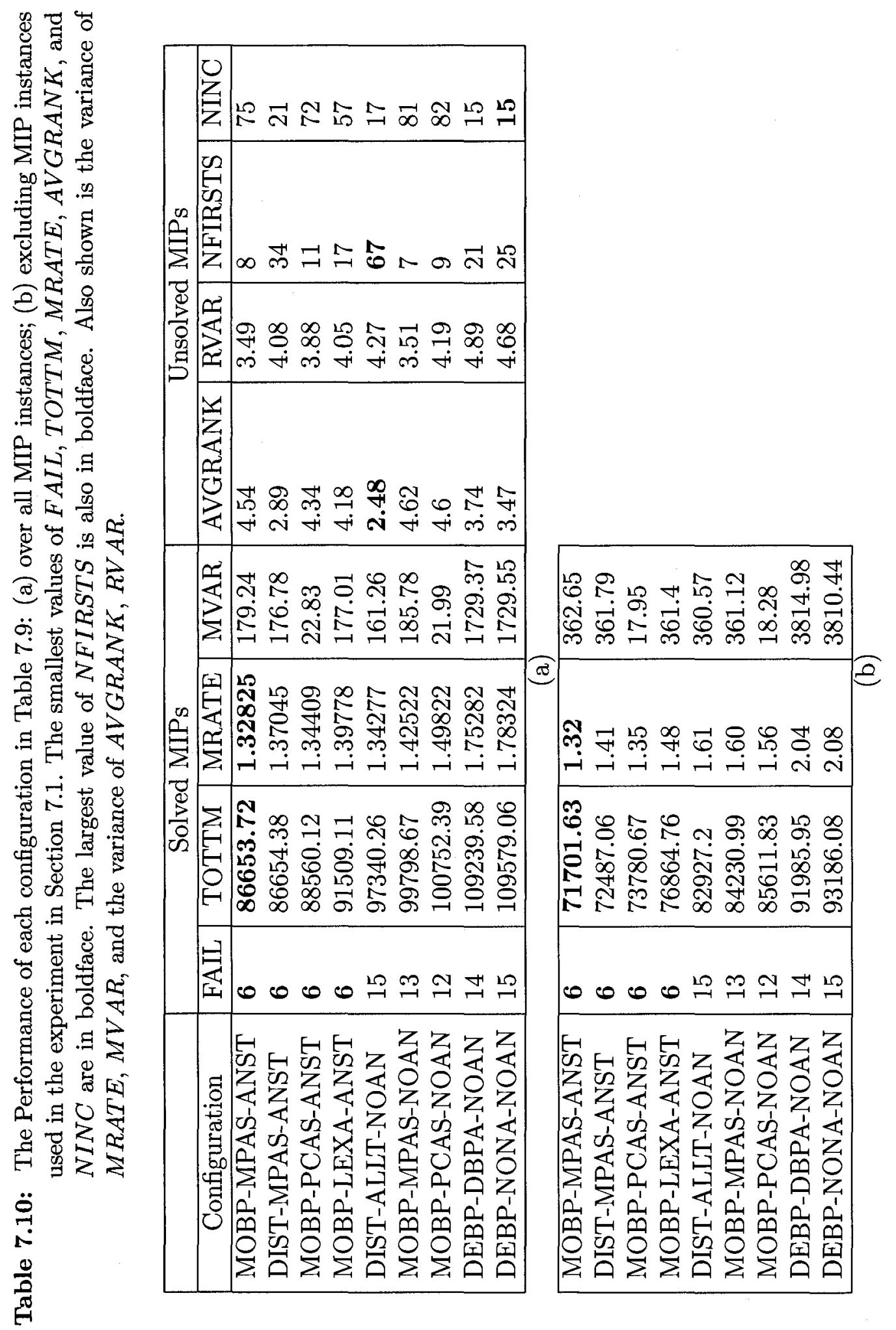


Table 7.11: The p-values of the ANOVA tests of $M R A T E$ between the best state of the art configuration and each of the proposed configurations.

\begin{tabular}{|l|c|}
\hline DEBP-DBPA-NOAN vs. & p-value \\
\hline DIST-ALLT-NOAN & 0.07 \\
DIST-MPAS-ANST & 0.03 \\
MOBP-LEXA-ANST & 0.04 \\
MOBP-PCAS-NOAN & 0.27 \\
MOBP-PCAS-ANST & 0.02 \\
MOBP-MPAS-NOAN & 0.06 \\
MOBP-MPAS-ANST & 0.01 \\
\hline
\end{tabular}

Figure 7.9 shows $M R A T E, r_{p 10}$, and $r_{p 90}$ for the configurations in Table 7.10. The difference between $r_{p 90}$ and $r_{p 10}$ is smallest for the MOBP-MPAS-ANST, DISTMPAS-ANST, and MOBP-PCAS-ANST configurations which correspond to the three smallest values of TOTTM.

Figure 7.10 shows the performance profiles, described in Section 6.3, of the state of the art configurations: DEBP-NONA-NOAN and DEBP-DBPA-NOAN, and the two best proposed configurations with respect to TOTTM: MOBP-MPAS-ANST and DIST-MPAS-ANST. From this plot, it can be seen that for any given value of $t_{M I} / t_{M I B}$ defined in Section 6.3, the fraction of MIP instances with a ratio less than this value for MOBP-MPAS-ANST and DIST-MPAS-ANST is greater than that for DEBP-NONA-ANST and DEBP-DBPA-ANST. For example, $t_{M I} / t_{M I B}$ for MOBP-MPAS-ANST is less than 2 for about $85 \%$ of the MIP instances; for DISTMPAS-ANST, this ratio is less than 2 for about $83 \%$ of MIP instances; for DEBPDBPA-NOAN, this ratio is less than 2 for about $71 \%$ of MIP instances; for DEBPNONA-NOAN, this ratio is less than 2 for about $69 \%$ of MIP instances. Over all of the MIP instances not solved within 10 seconds for every contending method: MOBPMPAS-ANST performed best for solving $11 \%$ of these instances, DIST-MPAS-ANST performed best for solving $22 \%$ of these instances, DEBP-DBPA-NOAN performed best for solving $7 \%$ of these instances, and DEBP-NONA-NOAN performed best for 


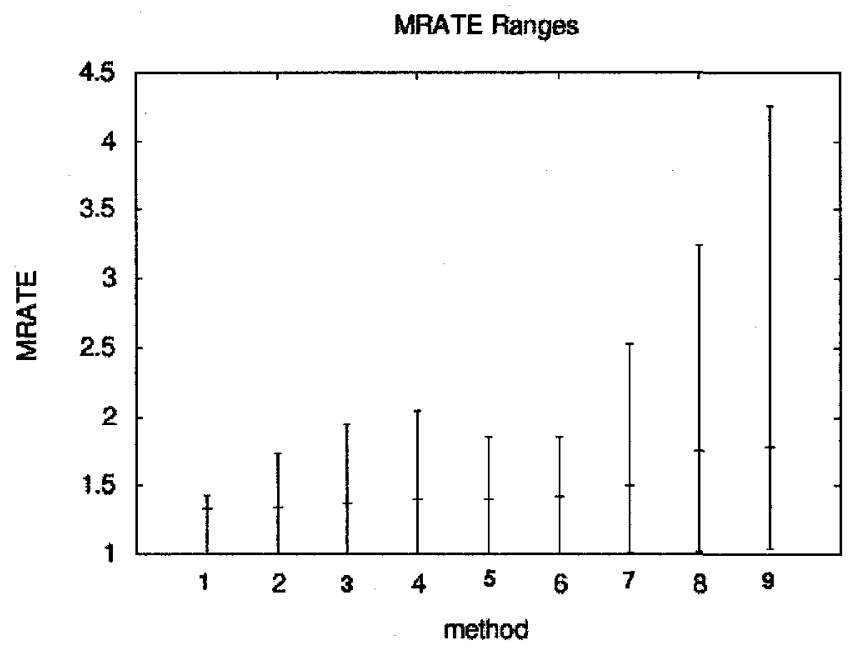

Figure 7.9: A plot showing MRATE, $r_{p 10}$, and $r_{p 90}$ for the following configurations: method $1=$ MOBP-MPAS-ANST, method $2=$ MOBP-PCAS-ANST, method $3=$ DIST-MPAS-ANST, method $4=$ MOBP-LEXA-ANST, method 5: DISTALLT-NOAN, method 6: MOBP-MPAS-NOAN, method 7: MOBP-PCASNOAN, method 8: DEBP-DBPA-NOAN, method 9: DEBP-NONA-NOAN.

solving $0 \%$ of these instances. Over all of the MIP instances not solved within 10 seconds for every contending method: MOBP-MPAS-ANST and DIST-MPAS-ANST solved $92 \%$ of these instances, DEBP-DBPA-NOAN solved $81 \%$ of these instances, and DEBP-NONA-NOAN solved $79 \%$ of these instances.

130 MIP instances were not solved to optimality within the one hour time limit, but at least one configuration was able to find a feasible solution. These instances are listed in Table A.5. With respect to AVGRANK and NFIRSTS, DIST-ALLTNOAN is the best configuration, the second best being DIST-MPAS-ANST. These results suggest that the DIST backtracking node selection method performs better than the other methods for either finding good MIP feasible solutions to the more difficult MIP instances or reducing the optimality gap of these MIP instances.

The best configurations with respect to NINC are DEBP-DBPA-NOAN and 


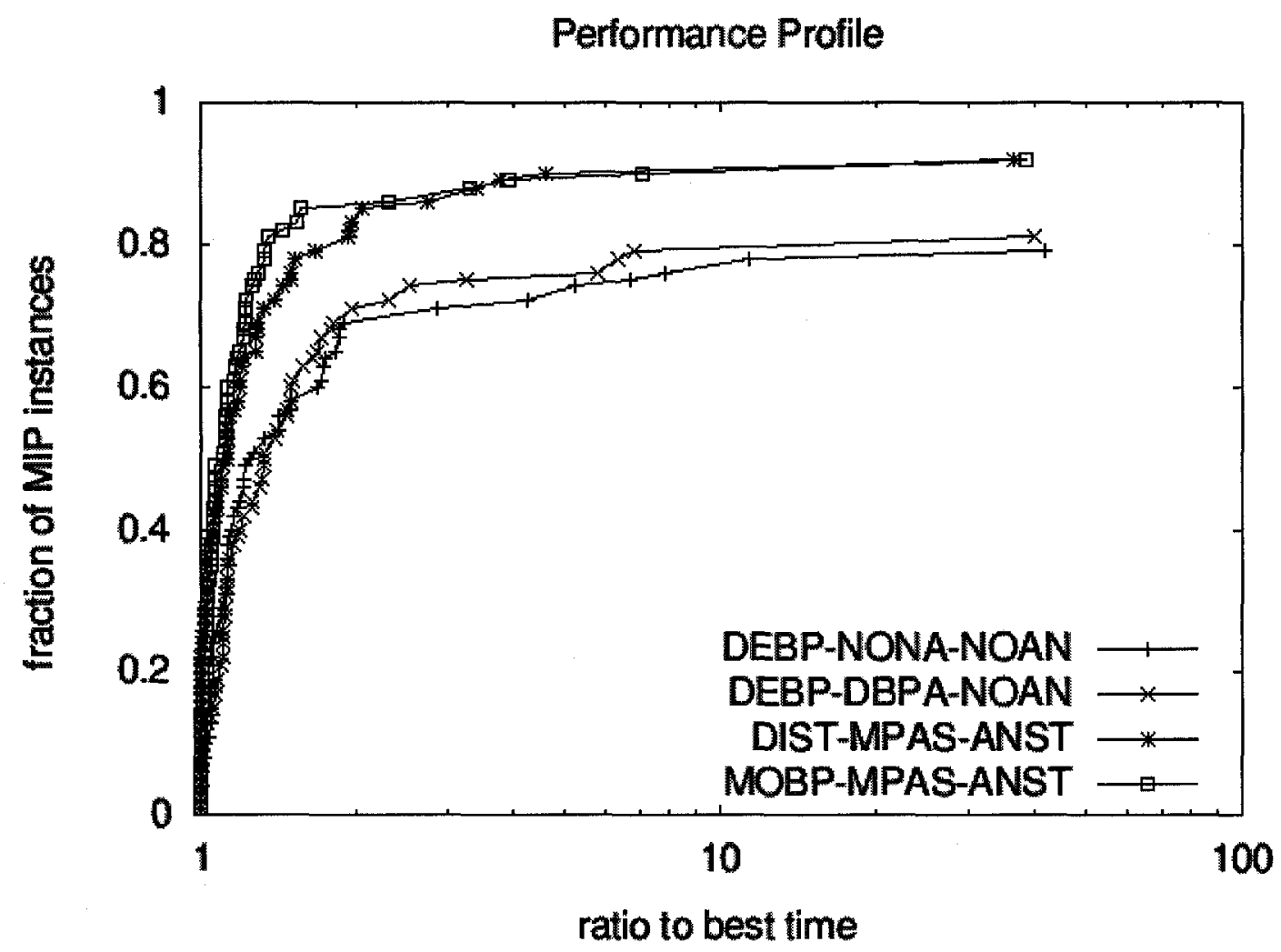

Figure 7.10: The performance profile comparing the two best proposed configurations with the two state of the art methods. Each point in the plot represents the fraction of MIP instances for which the ratio of time to solve them using the given configuration to best time to solve them over all the configurations in Table 7.9 is less than or equal to its value at that point. 
DEBP-NONA-NOAN because both of these configurations use the most-feasible backtracking node selection method without an aspiration value until an incumbent solution is found. The goal of the most-feasible backtracking node selection method is to quickly find a MIP feasible solution.

The results of this experiment suggest that DIST-MPAS-ANST is the best configuration for solving MIPs. It has the smallest number of unsolved MIPs (the same as 3 other configurations) and it is the only proposed configuration that outperforms the state of the art configurations with respect to all but one of the metrics: FAIL, TOTTM, MRATE, AVGRANK, and NFIRSTS. DIST-MPAS-ANST is only marginally worse than the only configuration, MOBP-MPAS-ANST, that performs better with respect to TOTTM. Similarly, DIST-MPAS-ANST is marginally worse than the best two configurations, MOBP-MPAS-ANST and MOBP-PCASANST with respect to MRATE. Finally, DIST-MPAS-ANST performs better than both MOBP-MPAS-ANST and MOBP-PCAS-ANST with respect to AVGRANK and NFIRSTS.

The complete details and results of this experiment are given in Appendix B.4.

\subsection{Problem-specific Node Selection}

Different MIP solver configurations may perform exceptionally well on particular classes of MIP models. A preliminary analysis was undertaken to determine whether some classes of MIP models are particularly well solved by the new solver configurations. The MOBP-MPAS-ANST and DIST-ALLT-NOAN configurations perform exceptionally well on Fixed Charge Transportation Problems (FCTP). There may well be other special cases that are handled well by some of the other new configurations, but this has been left for future research. The results for the FCTP follow below.

The goal of a FCTP [32] is to determine what routes should be used to transport goods from supply locations to demand locations while minimizing 
- the total of the costs that is associated with the amount of flow on each route, plus

- the total of the fixed costs associated with using each route.

Table 7.12 shows that MOPB-MPAS-ANST performs as well as or better than either state of the art configuration with respect to the amount of time required to solve the FCTPs. MOBP-MPAS-ANST reduced the time to solve three of the FCTPS by at least 15 minutes each, in one case reducing the time by over 45 minutes. Two of these FCTPs were not solved within the time limit using either state of the art configuration.

Table 7.13 shows that DIST-ALLT-NOAN performs as well as or better than either state of the art configuration with respect to the best incumbent solution found for all but one of the unsolved FCTPs. The best incumbent solutions found when using DIST-ALLT-NOAN are, on average, $27 \%$ less than the best incumbent solution found using either state of the art methods.

These results suggest that if the goal is to solve an FCTP to optimality, then it is probably best to use the MOBP-MPAS-ANST node selection configuration. If the goal is to find a good feasible solution to an FCTP given a limited amount of time, then it is probably best to use the DIST-ALLT-NOAN node selection configuration. 


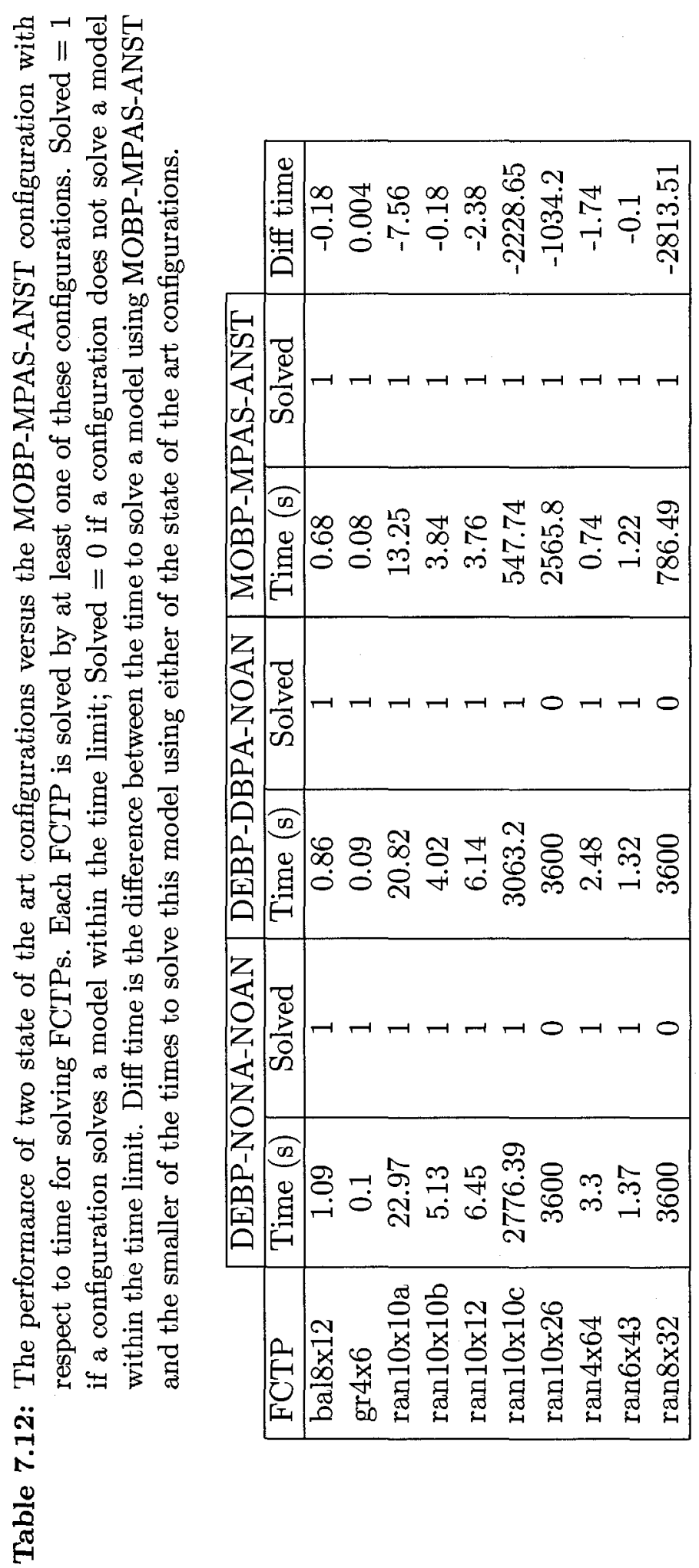



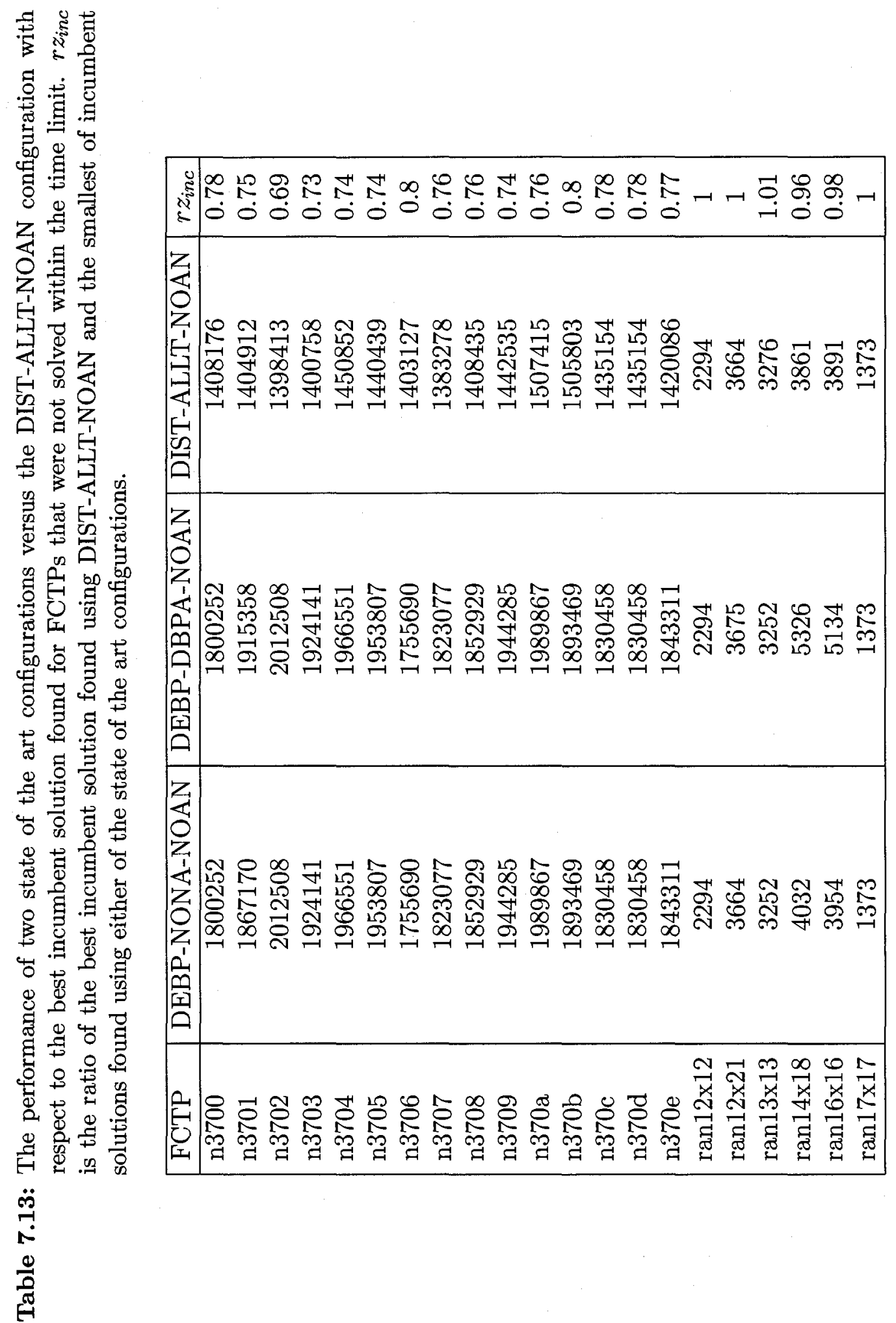


\section{Chapter 8}

\section{Conclusions}

1. The goal stated in Chapter 4 has been achieved: as shown in Chapters 5 and 7 , node selection methods that reduce the amount of effort required to solve MIPs have been developed.

2. The correlation between the objective values and numbers of candidate variables, as well as between the depths and numbers of candidate variables of nodes in the tree, can be exploited by backtracking node selection and backtrack triggering methods to reduce the amount of effort required to solve MIPs. This is shown in Sections 5.1, 5.3, 7.1 and 7.2.

3. For backtracking node selection, balancing the goals of MIP feasibility and MIP optimality using distributions of the objective values and the numbers of candidate variables of nodes in the tree reduces the amount of effort required to solve MIPs. This is shown in Sections 5.2, 7.1 and 7.2.

4. The amount of effort required to carry out a complex backtracking node selection algorithm can be large enough compared to the other branch and bound operations that it requires less effort to solve a MIP using a simpler node selection algorithm, as shown in Section 5.4.

5. The backtracking node selection method has much more influence on the amount 
of effort required to solve MIPs than the backtrack triggering method. This is shown in Section 7.1.

\subsection{Contributions}

1. Developed novel backtracking node selection heuristics that improve the performance of the branch and bound method for solving MIPs.

2. Developed novel methods of estimating the optimal objective value that, when used as an aspiration value to trigger backtracking, improve the performance of the branch and bound method for solving MIPs.

3. Introduced the concept of using probability distributions for the purpose of ordering nodes for selection during backtracking to improve the performance of solving MIPs.

4. Improved the best-projection backtrack triggering and backtracking node selection method by developing a method of computing projections without using an incumbent solution.

5. Introduced the concept of using the correlation between the objective values and numbers of candidate variables for the purpose of triggering backtracking and ordering nodes for selection during backtracking to improve the performance of solving MIPs.

6. Introduced the concept of the cost versus benefit analysis of backtracking node selection methods for the purpose of ordering node selection to improve the performance of solving MIPs.

7. Introduced the concept of using the correlation between the numbers of candidate variables and depths of nodes to estimate the depth of MIP feasible solutions. 
8. Introduced the concept of predicting the optimal objective value using the estimated depth of a MIP optimal node.

9. Performed an extensive computational study comparing 79 configurations of backtracking node selection methods, backtrack triggering methods, and active node search thresholds.

\subsection{Future Research}

1. The modified best-projection method presented in Section 5.1 may be improved by adaptively selecting the points $\left(z_{i}, c_{i}\right)$ to be used to estimate the degradation in objective value per unit MIP infeasibility. The change in the gradient of $z^{\text {min }}(c)$ may be useful towards this end.

2. The distribution method presented in Section 5.2 may be improved by using distribution functions that are better suited for modelling the objective values and the numbers of candidate variables of nodes in the tree. These distribution functions may incorporate the correlation of these values. Other node characteristics, such as depth, may also be used.

3. The distribution method may be improved by combining correlation information on the paths from the root node to each active node with global information such as the averages and variances of the objective values, numbers of candidate variables, and depths of nodes.

4. The parameters of the distribution functions used in the distribution method are computed using the assumption that the objective value of a node is independent of the objective value of any other node. This is not true for nodes that are ancestors or descendants of each other so it may be better to use only nodes that are not ancestors or descendants of each other in the computation of the parameters of the distribution functions. 
5. The ANST value may be improved if it is computed for each MIP instance based on some features of that instance and its branch and bound tree. Features that may be useful are the number of variables, the number of constraints, the number of active nodes, the optimality gap, the number of times backtracking has been performed, the number of MIP feasible nodes, and the number LP infeasible nodes. It may be possible to set the threshold value adaptively as the branch and bound method proceeds based on these features.

6. In Section 5.4 two methods of storing the active nodes in a branch and bound tree are discussed: the last active node created is placed at the end of the list; or each node is placed in the list by priority according to the node selection criteria. An empirical study comparing these methods has not been found in the literature. Such a comparative study should be conducted to determine which method is better.

7. The modified best-projection aspiration algorithm presented in Section 5.1.1 updates the aspiration value by searching through all of the active nodes after each node has been solved. If the number of active nodes becomes large then it may be better to reduce the frequency at which the aspiration value is updated.

8. The feasibility depth extrapolation aspiration algorithm presented in Section 5.3.1 estimates the depth of an optimal node as the smallest extrapolated depth of a MIP feasible node over all solved nodes. Therefore this estimate may become much too small without any way of increasing it. It may be better to increase this estimate using information such as the depths of all active nodes, or to use a different method of choosing the MIP optimal depth from all of the extrapolated depths of MIP feasible nodes. 


\section{References}

[1] ABACUS A Branch-And-CUt System Version 3.0 Users Guide and Reference Manual, 2007.

[2] T. Achterberg, T. Koch, and A. Martin. Branching rules revisited. Operations Research Letters, 33:42-54, 2005.

[3] Tobias Achterberg. Constraint Integer Programming. PhD thesis, Technische Universität Berlin, 2007. http://opus.kobv.de/tuberlin/volltexte/2007/1611/.

[4] Tobias Achterberg. Scip 1.0.0. http://scip.zib.de/, 2007.

[5] Tobias Achterberg, Thorsten Koch, and Alexander Martin. MIPLIB 2003. Operations Research Letters, 34(4):1-12, 2006. See http://miplib.zib.de.

[6] A. Atamtürk and M. Savelsbergh. Integer-programming software systems. Research Report BCOL.03.01, IEOR, University of California at Berkeley, 2005.

[7] M. Bénichou, J.M. Gauthier, P. Girodet, G. Hentges, G. Ribière, and O. Vincent. Experiments in mixed-integer linear programming. Mathematical Programming, 1:76-94, 1971.

[8] Timo Berthold. Primal Heuristics for Mixed Integer Programs. PhD thesis, Fachbereich Mathematik der Technische Universität Berlin, 2006.

[9] R. E. Bixby, M. Fenelon, Z. Gu, E. Rothberg, and R. Wunderling. Mip: Theory and practice - closing the gap. In M. Powell and S. Scholtes, editors, Systems 
Modelling and Optimization: Methods, Theory, and Applications, pages 19-49. Kluwer Academic Publisher, 2000.

[10] Robert E. Bixby, Sebastian Ceria, Cassandra M. McZeal, and Martin W.P. Savelsbergh. Miplib 2.0 - mixed integer problem library. http://miplib.zib.de/miplib3/miplib_prev.html, 1996.

[11] Robert E. Bixby, Sebastian Ceria, Cassandra M. McZeal, and Martin W.P. Savelsbergh. Miplib 3.0 - mixed integer problem library. http://miplib.zib.de/miplib3/miplib.html, 1996.

[12] R. J. Dakin. A tree search algorithm for mixed programming problems. Computer Journal, 8:250-255, 1965.

[13] Elizabeth D. Dolan and Jorge J. More. Benchmarking optimization software with performance profiles. Mathematical Programming, 91(2):201 - 213, 2002.

[14] N. J. Driebeek. An algorithm for the solution of mixed-integer programming problems. Management Science, 12:576-587, 1966.

[15] J. J. H. Forrest, J. P. H. Hirst, and J. A. Tomlin. Practical solution of large scale mixed integer programming problems with umpire. Management Science, 20:736-773, 1974.

[16] John Forrest and Robin Lougee-Heimer. Coin-or branch and cut 2.0.0, 2005. https://projects.coin-or.org/Cbc.

[17] J. M. Gauthier and G. Ribière. Experiments in mixed-integer linear programming using pseudocosts. Mathematical Programming, 12:26-47, 1977.

[18] Lou Hafer. Bonsaig algorithms \& design v2.8. Technical report, School of Computer Science, Simon Fraser University, December 1999.

[19] J. P. H. Hirst. Features required in branch and bound algorithms for (0-1) mixed integer linear programming. Privately circulated manuscript, December 1969. 
[20] John K. Karlof. Integer Programming: Theory and Practice. CRC Press, 2006.

[21] Konstantinos Kostikas and Charalambos Fragakis. Genetic programming applied to mixed integer programming. In Maarten Keijzer, Una-May O'Reilly, Simon M. Lucas, Ernesto Costa, and Terence Soule, editors, Genetic Programming, 7th European Conference, EuroGP2004, Coimbra, Portugal, April 5-7, 2004, Proceedings, volume 3003 of Lecture Notes in Computer Science. Springer, 2004 .

[22] Konstantinos Kostikas and Charalambos Fragakis. Genetic programming for guiding branch and bound search, 2004.

[23] H. Lee, J.M. Pinto, I.E. Grossmann, and S. Park. Mixed-integer linear programming model for refinery short-term scheduling of crude oil unloading with inventory management. Industrial and Engineering Chemistry Research, 35(5):1630$1641,1996$.

[24] J. Linderoth. Coral - mixed integer programming instances. http://coral.ie.lehigh.edu/mip-instances/, 2006.

[25] J. T. Linderoth and T. K. Ralphs. Noncommercial software for mixedinteger linear programming. Technical report, Department of Industrial and Systems Engineering, Lehigh University, Bethlehem, PA, 2004. http://www.lehigh.edu/ tkr2/research/papers/MILP04.pdf.

[26] J. T. Linderoth and M. W. P. Savelsbergh. A computational study of search strategies for mixed integer programming. INFORMS Journal on Computing, 11(2):173-187, 1999.

[27] A. Makhorin. Glpk 4.9. http://www.gnu.org/software/glpk/glpk.html, 2006.

[28] A. Martin. Integer programs with block structure. Technical report, Habilitations-Schrift, Technische Universität Berlin, 1998. http://www.zib.de/Publications/abstracts/SC-99-03/. 
[29] G. Mitra. Investigation of some branch and bound strategies for the solution of mixed integer linear programs. Mathematical Programming, 4:155-170, 1973.

[30] H. Mittelmann. Mixed integer linear programming benchmark (free codes). http://plato.asu.edu/ftp/milpf.html, February 2008.

[31] Ralf Niemann and Peter Marwedel. An algorithm for hardware/software partitioning using mixed integer linear programming. Design Automation for Embedded Systems, 2(2):165-193, March 1997.

[32] Udatta S. Palekar, Mark H. Karwan, and Stanley Zionts. A branch-and-bound method for the fixed charge transportation problem. Management Science, 36(9):1092-1105, 1990.

[33] J. Patel and J. W. Chinneck. Active-constraint variable ordering for faster feasibility of mixed integer linear programs. Mathematical Programming Series A, $110: 445-474,2007$.

[34] T.K. Ralphs and M. Güzelsoy. SYMPHONY 5.1.7 User's Manual, 2007.

[35] T.K. Ralphs and L. Ladányi. COIN/BCP Users Manual, January 2001.

[36] Arthur Richards, Tom Schouwenaars, Jonathan P. How, and Eric Feron. Spacecraft trajectory planning with avoidance constraints using mixed-integer linear programming. Journal of Guidance, Control, and Dynamics, 25(4):755-764, 2002.

[37] Martin W.P. Savelsbergh and George L. Nemhauser. Functional description of minto, a mixed integer optimizer, Technical report, Georgia Institute of Technology School of Industrial and Systems Engineering, Atlanta, GA 30332-205 USA, March 1998.

[38] J. A. Tomlin. Branch and bound methods for integer and non-convex programming. In J. Abadie, editor, Integer and Nonlinear Programming, pages 437-450. North-Holland, Amsterdam, 1970. 


\section{Appendix A}

\section{MIP Instances}

The number of MIP instances used to evaluate the performance of a branch and bound method should be large enough to obtain a sufficiently accurate measure of performance while also being small enough so that all of these instances can be solved within a reasonable amount of time. Table A.1 shows the MIP instances used in the branch and bound experiments performed in this thesis along with some of the formulation characteristics that are described below.

Name: The name of a MIP instance.

Cols: The number of variables.

Real: The number of real variables.

Int: The number of non-binary integer variables.

Bin: The number of binary variables.

Rows: The number of constraints.

Ineq: The number of inequality constraints.

Eq: The number of equality constraints.

NZ: The number of non-zero constraint coefficients. 
Table A.1: List of MIP instances taken from MIPLIB 2003, MIPLIB 3.0, MIPLIB 2.0 and CORAL with primal LP and MIP formulation characteristics.

\begin{tabular}{|c|c|c|c|c|c|c|c|c|}
\hline Name & Cols & Real & Int & $\operatorname{Bin}$ & Rows & Ineq & Eq & $\mathrm{NZ}$ \\
\hline 22433 & 429 & 198 & 0 & 231 & 199 & 0 & 198 & 3409 \\
\hline 23588 & 368 & 137 & 0 & 231 & 138 & 0 & 137 & 3702 \\
\hline 10teams & 2025 & 225 & 0 & 1800 & 231 & 95 & 135 & 14175 \\
\hline a1c1s1 & 3648 & 3456 & 192 & 0 & 3313 & 2064 & 1248 & 11618 \\
\hline aflow30a & 842 & 421 & 0 & 421 & 480 & 421 & 58 & 2512 \\
\hline aflow $40 \mathrm{~b}$ & 2728 & 1364 & 0 & 1364 & 1443 & 1364 & 78 & 8147 \\
\hline air01 & 771 & 0 & 0 & 771 & 24 & 0 & 23 & 4986 \\
\hline air02 & 6774 & 0 & 0 & 6774 & 51 & 0 & 50 & 68329 \\
\hline air03 & 10757 & 0 & 0 & 10757 & 125 & 0 & 124 & 101785 \\
\hline air04 & 8904 & 0 & 0 & 8904 & 824 & 0 & 823 & 81869 \\
\hline air05 & 7195 & 0 & 0 & 7195 & 427 & 0 & 426 & 59316 \\
\hline air06 & 8627 & 0 & 0 & 8627 & 826 & 0 & 825 & 79433 \\
\hline aligninq & 1831 & 1 & 0 & 1830 & 341 & 339 & 1 & 15735 \\
\hline arki001 & 1388 & 850 & 123 & 415 & 1049 & 1028 & 20 & 20440 \\
\hline atlanta-ip & 48738 & 1965 & 106 & 46667 & 21733 & 14652 & 7080 & 288828 \\
\hline bal $8 \times 12$ & 192 & 96 & 0 & 96 & 117 & 96 & 20 & 576 \\
\hline bc & 1751 & 1268 & 0 & 483 & 1914 & 1128 & 785 & 276843 \\
\hline bc1 & 1751 & 1499 & 0 & 252 & 1914 & 1128 & 785 & 276843 \\
\hline bell3b & 133 & 62 & 32 & 39 & 124 & 123 & 0 & 441 \\
\hline bell4 & 117 & 53 & 30 & 34 & 106 & 105 & 0 & 385 \\
\hline bell5 & 104 & 46 & 28 & 30 & 92 & 91 & 0 & 340 \\
\hline bienst1 & 505 & 477 & 0 & 28 & 577 & 448 & 128 & 2185 \\
\hline bienst2 & 505 & 470 & 0 & 35 & 577 & 448 & 128 & 2185 \\
\hline binkar10_1 & 2298 & 2128 & 0 & 170 & 1027 & 11 & 1015 & 6238 \\
\hline bk 4 x3 & 24 & 12 & 0 & 12 & 20 & 12 & 7 & 72 \\
\hline blend2 & 353 & 89 & 33 & 231 & 275 & 185 & 89 & 1497 \\
\hline bm23 & 27 & 0 & 0 & 27 & 21 & 20 & 0 & 505 \\
\hline cap6000 & 6000 & 0 & 0 & 6000 & 2177 & 2053 & 123 & 54238 \\
\hline cracpb1 & 572 & 0 & 0 & 572 & 144 & 54 & 89 & 4730 \\
\hline d10200 & 2000 & 0 & 1267 & 733 & 948 & 747 & 200 & 59637 \\
\hline d20200 & 4000 & 0 & 819 & 3181 & 1503 & 1302 & 200 & 193389 \\
\hline dano3_3 & 13873 & 13804 & 0 & 69 & 3203 & 1978 & 1224 & 79656 \\
\hline dano3_4 & 13873 & 13781 & 0 & 92 & 3203 & 1978 & 1224 & 79656 \\
\hline dano3_5 & 13873 & 13758 & 0 & 115 & 3203 & 1978 & 1224 & 79656 \\
\hline dano3mip & 13873 & 13321 & 0 & 552 & 3203 & 1978 & 1224 & 79656 \\
\hline danoint & 521 & 465 & 0 & 56 & 665 & 520 & 144 & 3233 \\
\hline demulti & 548 & 473 & 0 & 75 & 291 & 212 & 78 & 1833 \\
\hline disctom & 10000 & 0 & 0 & 10000 & 400 & 0 & 399 & 40000 \\
\hline ds & 67732 & 0 & 67732 & 0 & 657 & 0 & 656 & 1091791 \\
\hline dsbmip & 1886 & 1694 & 32 & 160 & 1855 & 1462 & 392 & 9768 \\
\hline egout & 141 & 86 & 0 & 55 & 99 & 55 & 43 & 392 \\
\hline eilD76 & 1898 & 0 & 1898 & 0 & 76 & 0 & 75 & 21009 \\
\hline enigma & 100 & 0 & 0 & 100 & 22 & 0 & 21 & 298 \\
\hline fast 0507 & 63009 & 0 & 0 & 63009 & 508 & 507 & 0 & 472358 \\
\hline fiball & 34219 & 1 & 258 & 33960 & 3708 & 478 & 3229 & 104793 \\
\hline fiber & 1298 & 44 & 0 & 1254 & 364 & 0 & 363 & 4198 \\
\hline fixnet3 & 878 & 500 & 0 & 378 & 479 & 378 & 100 & 2631 \\
\hline fixnet4 & 878 & 500 & 0 & 378 & 479 & 378 & 100 & 2621 \\
\hline fixnet6 & 878 & 500 & 0 & 378 & 479 & 378 & 100 & 2550 \\
\hline flugpl & 18 & 7 & 11 & 0 & 19 & 12 & 6 & 64 \\
\hline gen. & 870 & 720 & 6 & 144 & 781 & 630 & 150 & 3174 \\
\hline germanrr & 10813 & 239 & 5286 & 5288 & 10780 & 10541 & 238 & 175548 \\
\hline gesa2 & 1224 & 816 & 168 & 240 & 1393 & 1344 & 48 & 6000 \\
\hline gesa2-o & 1224 & 504 & 336 & 384 & 1249 & 1128 & 120 & 4608 \\
\hline glass4 & 322 & 20 & 0 & 302 & 397 & 360 & 36 & 1834 \\
\hline
\end{tabular}


Table A.1 - continued from previous page

\begin{tabular}{|c|c|c|c|c|c|c|c|c|}
\hline Name & Cols & Real & Int & Bin & Rows & Ineq & $\mathrm{Eq}$ & $\mathrm{NZ}$ \\
\hline gr4x6 & 48 & 24 & 0 & 24 & 35 & 24 & 10 & 144 \\
\hline gt2 & 188 & 0 & 164 & 24 & 30 & 29 & 0 & 468 \\
\hline haprp & 1828 & 0 & 1828 & 0 & 1049 & 60 & 988 & 5363 \\
\hline harp2 & 2993 & 0 & 0 & 2993 & 113 & 39 & 73 & 8833 \\
\hline $\operatorname{irp}$ & 20315 & 0 & 0 & 20315 & 40 & 1 & 38 & 118569 \\
\hline khb05250 & 1350 & 1326 & 0 & 24 & 102 & 24 & 77 & 3973 \\
\hline 1152lav & 1989 & 0 & 0 & 1989 & 98 & 1 & 96 & 11911 \\
\hline leo1 & 6731 & 1 & 0 & 6730 & 594 & 592 & 1 & 131219 \\
\hline leo2 & 11100 & 1 & 0 & 11099 & 594 & 592 & 1 & 219960 \\
\hline liu & 1156 & 67 & 0 & 1089 & 2179 & 2178 & 0 & 10627 \\
\hline $\operatorname{lp} 41$ & 1086 & 0 & 0 & 1086 & 86 & 1 & 84 & 5763 \\
\hline $\operatorname{lnn}$ & 7253 & 4798 & 0 & 2455 & 8702 & 7692 & 1009 & 52643 \\
\hline lseu & 89 & 0 & 0 & 89 & 29 & 28 & 0 & 394 \\
\hline manna81 & 3321 & 0 & 3303 & 18 & 6481 & 6480 & 0 & 16281 \\
\hline markshare1 & 62 & 12 & 0 & 50 & 7 & 0 & 6 & 324 \\
\hline markshare2 & 74 & 14 & 0 & 60 & 8 & 0 & 7 & 448 \\
\hline $\operatorname{mas} 284$ & 151 & 1 & 0 & 150 & 69 & 68 & 0 & 9782 \\
\hline mas74 & 151 & 1 & 0 & 150 & 14 & 13 & 0 & 1857 \\
\hline $\operatorname{mas} 76$ & 151 & 1 & 0 & 150 & 13 & 12 & 0 & 1791 \\
\hline mcf2 & 521 & 465 & 0 & 56 & 665 & 520 & 144 & 3233 \\
\hline mcsched & 1747 & 2 & 14 & 1731 & 2108 & 1906 & 201 & 8089 \\
\hline misc01 & 83 & 1 & 0 & 82 & 55 & 37 & 17 & 746 \\
\hline $\operatorname{misc} 02$ & 59 & 1 & 0 & 58 & 40 & 25 & 14 & 414 \\
\hline misc03 & 160 & 1 & 0 & 159 & 97 & 69 & 27 & 2054 \\
\hline misc04 & 4897 & 4867 & 0 & 30 & 1726 & 1414 & 311 & 17253 \\
\hline $\operatorname{misc} 05$ & 136 & 62 & 0 & 74 & 301 & 271 & 29 & 2946 \\
\hline $\operatorname{misc} 06$ & 1808 & 1696 & 0 & 112 & 821 & 570 & 250 & 5860 \\
\hline $\operatorname{misc07}$ & 260 & 1 & 0 & 259 & 213 & 177 & 35 & 8620 \\
\hline mitre & 10724 & 0 & 0 & 10724 & 2055 & 1671 & 383 & 49028 \\
\hline mkc & 5325 & 2 & 0 & 5323 & 3412 & 3409 & 2 & 19984 \\
\hline $\mathrm{mkc1}$ & 5325 & 2238 & 0 & 3087 & 3412 & 3409 & 2 & 19984 \\
\hline $\bmod 008$ & 319 & 0 & 0 & 319 & 7 & 6 & 0 & 1562 \\
\hline $\bmod 010$ & 2655 & 0 & 0 & 2655 & 147 & 1 & 145 & 13858 \\
\hline $\bmod 011$ & 10958 & 10862 & 0 & 96 & 4481 & 113 & 4367 & 29839 \\
\hline $\bmod 013$ & 96 & 48 & $\mathbf{0}$ & 48 & 63 & 48 & 14 & 288 \\
\hline modglob & 422 & 324 & 0 & 98 & 292 & 196 & 95 & 1390 \\
\hline momentum1 & 5174 & 2825 & 0 & 2349 & 42681 & 42122 & 558 & 106005 \\
\hline momentum2 & 3732 & 1923 & 1 & 1808 & 24238 & 24017 & 220 & 353367 \\
\hline momentum3 & 13532 & 6933 & 1 & 6598 & 56823 & 55970 & 852 & 962826 \\
\hline msc98-ip & 21143 & 853 & 53 & 20237 & 15851 & 11549 & 4301 & 100358 \\
\hline mzzv11 & 10240 & 0 & 251 & 9989 & 9500 & 6127 & 3372 & 134808 \\
\hline $\operatorname{mzz} 42 z$ & 11717 & 0 & 235 & 11482 & 10461 & 5958 & 4502 & 151455 \\
\hline n3700 & 10000 & 5000 & 0 & 5000 & 5151 & 5000 & 150 & 30000 \\
\hline n3701 & 10000 & 5000 & 0 & 5000 & 5151 & 5000 & 150 & 30000 \\
\hline n3702 & 10000 & 5000 & 0 & 5000 & 5151 & 5000 & 150 & 30000 \\
\hline n3703 & 10000 & 5000 & 0 & 5000 & 5151 & 5000 & 150 & 29999 \\
\hline n3704 & 10000 & 5000 & 0 & 5000 & 5151 & 5000 & 150 & 30000 \\
\hline n3705 & 10000 & 5000 & 0 & 5000 & 5151 & 5000 & 150 & 30000 \\
\hline n3706 & 10000 & 5000 & 0 & 5000 & 5151 & 5000 & 150 & 30000 \\
\hline n3707 & 10000 & 5000 & 0 & 5000 & 5151 & 5000 & 150 & 30000 \\
\hline n3708 & 10000 & 5000 & 0 & 5000 & 5151 & 5000 & 150 & 30000 \\
\hline n3709 & 10000 & 5000 & 0 & 5000 & 5151 & 5000 & 150 & 30000 \\
\hline n370a & 10000 & 5000 & 0 & 5000 & 5151 & 5000 & 150 & 30000 \\
\hline n370b & 10000 & 5000 & 0 & 5000 & 5151 & 5000 & 150 & 30000 \\
\hline $\mathrm{n} 370 \mathrm{c}$ & 10000 & 5000 & 0 & 5000 & 5151 & 5000 & 150 & 30000 \\
\hline n370d & 10000 & 5000 & 0 & 5000 & 5151 & 5000 & 150 & 30000 \\
\hline n370e & 10000 & 5000 & 0 & 5000 & 5151 & 5000 & 150 & 30000 \\
\hline nag & 2884 & 1499 & 1385 & 0 & 5841 & 4465 & 1375 & 26500 \\
\hline
\end{tabular}


Table A.1 - continued from previous page

\begin{tabular}{|c|c|c|c|c|c|c|c|c|}
\hline Name & Cols & Real & Int & Bin & Rows & Ineq & Eq & NZ \\
\hline neos-430149 & 395 & 215 & 0 & 180 & 991 & 780 & 210 & 2910 \\
\hline neos- 476283 & 11915 & 6327 & 0 & 5588 & 10016 & 9951 & 64 & 3946449 \\
\hline neos- 480878 & 534 & 345 & 0 & 189 & 1322 & 1286 & 35 & 44826 \\
\hline neos- 494568 & 6889 & 153 & 0 & 6736 & 2216 & 2091 & 124 & 122257 \\
\hline neos-495307 & 9423 & 0 & 9423 & 0 & 4 & 3 & 0 & 37254 \\
\hline neos- 498623 & 9861 & 1361 & 0 & 8500 & 2048 & 1901 & 146 & 156991 \\
\hline neos-501453 & 165 & 18 & 147 & 0 & 41 & 26 & 14 & 685 \\
\hline neos-501474 & 206 & 59 & 147 & 0 & 266 & 251 & 14 & 2419 \\
\hline neos-503737 & 2850 & 350 & 0 & 2500 & 501 & 351 & 149 & 17200 \\
\hline neos-504674 & 844 & 694 & 0 & 150 & 1345 & 1094 & 250 & 3600 \\
\hline neos-504815 & 674 & 554 & 0 & 120 & 1068 & 867 & 200 & 2856 \\
\hline neos-506422 & 2527 & 2464 & 0 & 63 & 6812 & 6804 & 7 & 34083 \\
\hline neos-506428 & 42981 & 0 & 0 & 42981 & 129926 & 129831 & 94 & 343488 \\
\hline neos-512201 & 838 & 688 & 0 & 150 & 1338 & 1093 & 244 & 3568 \\
\hline neos-520729 & 91149 & 60441 & 0 & 30708 & 31179 & 40 & 31138 & 327996 \\
\hline neos-522351 & 1524 & 1284 & 0 & 240 & 1706 & 1609 & 96 & 5700 \\
\hline neos-525149 & 3640 & 40 & 0 & 3600 & 144121 & 144001 & 119 & 1522840 \\
\hline neos-530627 & 103 & 75 & 28 & 0 & 114 & 77 & 36 & 350 \\
\hline neos-538867 & 792 & 0 & 0 & 792 & 1171 & 1153 & 17 & 4554 \\
\hline neos-538916 & 864 & 0 & 0 & 864 & 1315 & 1297 & 17 & 4976 \\
\hline neos-544324 & 10080 & 0 & 0 & 10080 & 733 & 732 & 0 & 1767360 \\
\hline neos-547911 & 3528 & 0 & 0 & 3528 & 694 & 693 & 0 & 618576 \\
\hline neos-548047 & 2020 & 1 & 0 & 2019 & 3971 & 3970 & 0 & 26406 \\
\hline neos-548251 & 1922 & 992 & 0 & 930 & 2387 & 1456 & 930 & 5792 \\
\hline neos-551991 & 1730 & 1 & 0 & 1729 & 3333 & 3332 & 0 & 31632 \\
\hline neos-555001 & 3855 & 0 & 75 & 3780 & 3475 & 1270 & 2204 & 16657 \\
\hline neos-555298 & 4827 & 0 & 15 & 4812 & 2756 & 1336 & 1419 & 20153 \\
\hline neos-555343 & 3815 & 0 & 15 & 3800 & 3327 & 2599 & 727 & 16975 \\
\hline neos-555424 & 3815 & 0 & 15 & 3800 & 2677 & 2606 & 70 & 15675 \\
\hline neos-555694 & 4139 & 1320 & 0 & 2819 & 1949 & 1845 & 103 & 42443 \\
\hline neos-555771 & 4170 & 1338 & 0 & 2832 & 1979 & 1857 & 121 & 43255 \\
\hline neos-555884 & 3815 & 0 & 15 & 3800 & 4332 & 3086 & 1245 & 19075 \\
\hline neos-555927 & 1945 & 0 & 15 & 1930 & 1404 & 763 & 640 & 7973 \\
\hline neos-565672 & 190589 & 15228 & 0 & 175361 & 318335 & 318334 & 0 & 825044 \\
\hline neos-565815 & 1276 & 0 & 0 & 1276 & 15414 & 15372 & 41 & 124716 \\
\hline neos-570431 & 511 & 1 & 0 & 510 & 932 & 931 & 0 & 12042 \\
\hline neos-574665 & 740 & 492 & 64 & 184 & 3791 & 3335 & 455 & 16796 \\
\hline neos-578379 & 17010 & 0 & 0 & 17010 & 21703 & 10720 & 10982 & 101560 \\
\hline neos-582605 & 1265 & 865 & 0 & 400 & 1241 & 836 & 404 & 3755 \\
\hline neos-583731 & 1350 & 0 & 0 & 1350 & 1492 & 1471 & 20 & 5310 \\
\hline neos-584146 & 811 & 561 & 0 & 250 & 937 & 636 & 300 & 3056 \\
\hline neos- 584851 & 445 & 40 & 0 & 405 & 662 & 658 & 3 & 1734 \\
\hline neos-584866 & 3674 & 90 & 0 & 3584 & 9010 & 9001 & 8 & 21506 \\
\hline neos-585192 & 2597 & 1553 & 0 & 1044 & 2629 & 1081 & 1547 & 72401 \\
\hline neos-585467 & 2116 & 1270 & 0 & 846 & 2167 & 901 & 1265 & 50062 \\
\hline neos-593853 & 2400 & 1200 & 0 & 1200 & 1607 & 1407 & 199 & 7200 \\
\hline neos-595904 & 4508 & 3360 & 0 & 1148 & 2453 & 2300 & 152 & 26872 \\
\hline neos-595905 & 1200 & 888 & 0 & 312 & 705 & 628 & 76 & 6988 \\
\hline neos-595925 & 1276 & 840 & 0 & 436 & 957 & 872 & 84 & 7236 \\
\hline neos-598183 & 1696 & 1260 & 0 & 436 & 993 & 876 & 116 & 10084 \\
\hline neos-603073 & 1696 & 1260 & 0 & 436 & 993 & 876 & 116 & 10084 \\
\hline neos-611135 & 6400 & 0 & 0 & 6400 & 5278 & 5246 & 31 & 775700 \\
\hline neos-611838 & 9954 & 9900 & 0 & 54 & 1877 & 1132 & 744 & 46981 \\
\hline neos- 612125 & 9554 & 9504 & 0 & 50 & 1796 & 1051 & 744 & 45345 \\
\hline neos-612143 & 9832 & 9780 & 0 & 52 & 1843 & 1098 & 744 & 46475 \\
\hline neos-612162 & 9893 & 9840 & 0 & 53 & 1860 & 1115 & 744 & 46728 \\
\hline neos-619167 & 3452 & 3052 & 0 & 400 & 6801 & 6641 & 159 & 20021 \\
\hline neos-631164 & 1282 & 245 & 0 & 1037 & 407 & 194 & 212 & 3401 \\
\hline
\end{tabular}


Table A.1 - continued from previous page

\begin{tabular}{|c|c|c|c|c|c|c|c|c|}
\hline Name & Cols & Real & Int & $\mathrm{Bin}$ & Rows & Ineq & $\mathrm{Eq}$ & NZ \\
\hline neos-631517. & 1090 & 231 & 0 & 859 & 352 & 185 & 166 & 2974 \\
\hline neos- 631694 & 3725 & 0 & 0 & 3725 & 3997 & 3921 & 75 & 18573 \\
\hline neos-631709 & 45150 & 0 & 0 & 45150 & 46497 & 46346 & 150 & 225448 \\
\hline neos-631710 & 167056 & 0 & 0 & 167056 & 169577 & 169276 & 300 & 834722 \\
\hline neos-631784 & 22725 & 0 & 0 & 22725 & 23997 & 23921 & 75 & 113323 \\
\hline neos-632335 & 12719 & 0 & 0 & 12719 & 24865 & 12168 & 12696 & 84663 \\
\hline neos- 633273 & 11154 & 0 & 0 & 11154 & 21782 & 10649 & 11132 & 74074 \\
\hline neos-641591 & 18235 & 0 & 328 & 17907 & 1086 & 758 & 327 & 218290 \\
\hline neos-655508 & 13572 & 0 & 0 & 13572 & 13574 & 13457 & 116 & 53824 \\
\hline neos-662469 & 18235 & 0 & 328 & 17907 & 1086 & 758 & 327 & 218290 \\
\hline neos-686190 & 3660 & 0 & 60 & 3600 & 3665 & 3544 & 120 & 21625 \\
\hline neos-691058 & 3006 & 1251 & 0 & 1755 & 2668 & 2206 & 461 & 30912 \\
\hline neos- 691073 & 1935 & 180 & 0 & 1755 & 2668 & 2206 & 461 & 29841 \\
\hline neos-693347 & 1576 & 171 & 0 & 1405 & 3193 & 2861 & 331 & 113538 \\
\hline neos-702280 & 7199 & 0 & 0 & 7199 & 1601 & 1600 & 0 & 2429081 \\
\hline neos-709469 & 224 & 0 & 0 & 224 & 470 & 413 & 56 & 4656 \\
\hline neos-717614 & 3049 & 49 & 2916 & 84 & 892 & 296 & 595 & 13526 \\
\hline neos-738098 & 9093 & 147 & 0 & 8946 & 25850 & 25353 & 496 & 101507 \\
\hline neos-775946 & 4710 & 1593 & 0 & 3117 & 6603 & 6475 & 127 & 111313 \\
\hline neos-777800 & 6400 & 0 & 0 & 6400 & 480 & 478 & 1 & 38400 \\
\hline neos-780889 & 182700 & 0 & 0 & 182700 & 73911 & 351 & 73559 & 621460 \\
\hline neos-785899 & 1320 & 0 & 0 & 1320 & 1654 & 1653 & 0 & 18380 \\
\hline neos-785912 & 1380 & 0 & 0 & 1380 & 1715 & 1714 & 0 & 17870 \\
\hline neos-785914 & 1260 & 0 & 0 & 1260 & 1591 & 1590 & 0 & 16430 \\
\hline neos-787933 & 236376 & 0 & 0 & 236376 & 1898 & 1897 & 0 & 300084 \\
\hline neos-791021 & 9448 & 0 & 0 & 9448 & 3695 & 970 & 2724 & 29732 \\
\hline neos-796608 & 311 & 192 & 119 & 0 & 287 & 95 & 191 & 873 \\
\hline neos-799711 & 41998 & 41088 & 0 & 910 & 59219 & 35827 & 23391 & 147165 \\
\hline neos-799716 & 30619 & 29709 & 0 & 910 & 40419 & 17031 & 23387 & 99929 \\
\hline neos- 799838 & 20844 & 0 & 0 & 20844 & 5977 & 73 & 5903 & 69804 \\
\hline neos-801834 & 3220 & 0 & 0 & 3220 & 3301 & 3241 & 59 & 58420 \\
\hline neos-803219 & 640 & 580 & 0 & 60 & 902 & 431 & 470 & 3070 \\
\hline neos- 803220 & 630 & 570 & 0 & 60 & 892 & 421 & 470 & 3030 \\
\hline neos-806323 & 1060 & 950 & 0 & 110 & 1542 & 691 & 850 & 5740 \\
\hline neos-807454 & 1638 & 0 & 0 & 1638 & 1623 & 1342 & 280 & 35323 \\
\hline neos-807456 & 1635 & 0 & 0 & 1635 & 841 & 0 & 840 & 6540 \\
\hline neos-807639 & 1030 & 950 & 0 & 80 & 1542 & 691 & 850 & 5580 \\
\hline neos-807705 & 1030 & 950 & 0 & 80 & 1542 & 691 & 850 & 5580 \\
\hline neos-808072 & 1702 & 1 & 0 & 1701 & 1714 & 1468 & 245 & 38107 \\
\hline neos-808214 & 1308 & 0 & 0 & 1308 & 641 & 437 & 203 & 22583 \\
\hline neos-810286 & 2915 & 0 & 0 & 2915 & 2676 & 2296 & 379 & 71666 \\
\hline neos-810326 & 1702 & 1 & 0 & 1701 & 1750 & 1468 & 281 & 39843 \\
\hline neos1 & 2112 & 0 & 0 & 2112 & 5021 & 4921 & 99 & 21600 \\
\hline neos10 & 23489 & 0 & 5 & 23484 & 46794 & 46777 & 16 & 251230 \\
\hline neos11 & 1220 & 320 & 0 & 900 & 2707 & 2471 & 235 & 9540 \\
\hline neos12 & 3983 & 847 & 0 & 3136 & 8318 & 7743 & 574 & 26229 \\
\hline neos13 & 1827 & 12 & 0 & 1815 & 20853 & 20852 & 0 & 253854 \\
\hline neos14 & 792 & 656 & 0 & 136 & 553 & 313 & 239 & 2318 \\
\hline neos15 & 792 & 632 & 0 & 160 & 553 & 313 & 239 & 2318 \\
\hline neos16 & 377 & 0 & 41 & 336 & 1019 & 1009 & 9 & 2802 \\
\hline neos17 & 535 & 235 & 0 & 300 & 487 & 485 & 1 & 5416 \\
\hline neos18 & 3312 & 0 & 0 & 3312 & 11403 & 9008 & 2394 & 24694 \\
\hline neos19 & 103789 & 93789 & 0 & 10000 & 34083 & 91 & 33991 & 198442 \\
\hline neos2 & 2101 & 1061 & 0 & 1040 & 1104 & 1060 & 43 & 7330 \\
\hline neos20 & 1165 & 198 & 111 & 856 & 2447 & 1693 & 753 & 7433 \\
\hline neos3 & 2747 & 1387 & 0 & 1360 & 1443 & 1386 & 56 & 9585 \\
\hline neos 4 & 22884 & 5712 & 0 & 17172 & 38578 & 37441 & 1136 & 116040 \\
\hline neos5 & 63 & 10 & 0 & 53 & 64 & 63 & 0 & 2079 \\
\hline
\end{tabular}


Table A.1 - continued from previous page

\begin{tabular}{|c|c|c|c|c|c|c|c|c|}
\hline Name & Cols & Real & Int & Bin & Rows & Ineq & $\mathrm{Eq}$ & $\overline{N Z}$ \\
\hline neos6 & 8786 & 446 & 0 & 8340 & 1037 & 813 & $\overline{223}$ & 252169 \\
\hline neos7 & 1556 & 1102 & 30 & 424 & 1995 & 1525 & 469 & 5472 \\
\hline neos8 & 23228 & 0 & 4 & 23224 & 46325 & 46312 & 12 & 313212 \\
\hline neos9 & 81408 & 79309 & 0 & 2099 & 31601 & 23873 & 7727 & 264192 \\
\hline net12 & 14115 & 12512 & 0 & 1603 & 14022 & 13469 & 552 & 80423 \\
\hline newdano & 505 & 449 & 0 & 56 & 577 & 448 & 128 & 2185 \\
\hline noswot & 128 & 28 & 25 & 75 & 183 & 180 & 2 & 760 \\
\hline npmv07 & 220686 & 218806 & 0 & 1880 & 76343 & 52641 & 23701 & 859615 \\
\hline nsa & 388 & 352 & 0 & 36 & 1298 & 1261 & 36 & 4205 \\
\hline nsrand-ipx & 6621 & 1 & 0 & 6620 & 736 & 735 & 0 & 223262 \\
\hline nug08 & 1632 & 0 & 1632 & 0 & 913 & 1 & 911 & 8304 \\
\hline nw04 & 87482 & 0 & 0 & 87482 & 37 & 0 & 36 & 724148 \\
\hline opt1217 & 769 & 1 & 0 & 768 & 65 & 16 & 48 & 1543 \\
\hline p0033 & 33 & 0 & 0 & 33 & 17 & 16 & 0 & 131 \\
\hline p0040 & 40 & 0 & 0 & 40 & 24 & 23 & 0 & 150 \\
\hline p0201 & 201 & 0 & 0 & 201 & 134 & 133 & 0 & 2124 \\
\hline p0282 & 282 & 0 & 0 & 282 & 242 & 241 & 0 & 2248 \\
\hline p0291 & 291 & 0 & 0 & 291 & 253 & 252 & 0 & 2321 \\
\hline p0548 & 548 & 0 & 0 & 548 & 177 & 176 & 0 & 2127 \\
\hline p2756 & 2756 & 0 & 0 & 2756 & 756 & 755 & 0 & 11103 \\
\hline p6b & 462 & 0 & 0 & 462 & 5853 & 5852 & 0 & 12166 \\
\hline pg & 2700 & 2600 & 0 & 100 & 126 & 25 & 100 & 7800 \\
\hline pg5_34 & 2600 & 2500 & 0 & 100 & 226 & 225 & 0 & 10300 \\
\hline pipex & 48 & 0 & 0 & 48 & 26 & 9 & 16 & 240 \\
\hline pk1 & 86 & 31 & $\mathbf{0}$ & 55 & 46 & 30 & 15 & 916 \\
\hline pp08a & 240 & 176 & 0 & 64 & 137 & 72 & 64 & 656 \\
\hline pp08aCUTS & 240 & 176 & 0 & 64 & 247 & 182 & 64 & 1015 \\
\hline prod1 & 250 & 101 & 0 & 149 & 209 & 201 & 7 & 5351 \\
\hline prod2 & 301 & 101 & 0 & 200 & 212 & 201 & 10 & 10502 \\
\hline protfold & 1835 & 0 & 0 & 1835 & 2113 & 2075 & 37 & 23611 \\
\hline qap10 & 4150 & 0 & 4150 & 0 & 1821 & 0 & 1820 & 20810 \\
\hline qiu & 840 & 792 & 0 & 48 & 1193 & 1060 & 132 & 3744 \\
\hline qnet1 & 1541 & 124 & 129 & 1288 & 504 & 172 & 331 & 4746 \\
\hline qnet1_o & 1541 & 124 & 129 & 1288 & 457 & 125 & 331 & 4338 \\
\hline ramos3 & 2187 & 0 & 0 & 2187 & 2188 & 2187 & 0 & 34992 \\
\hline $\operatorname{ran} 10 \times 10 a$ & 200 & 100 & 0 & 100 & 121 & 100 & 20 & 600 \\
\hline $\operatorname{ran} 10 \times 10 b$ & 200 & 100 & 0 & 100 & 121 & 100 & 20 & 600 \\
\hline $\operatorname{ran} 10 \times 10 c$ & 200 & 100 & 0 & 100 & 121 & 100 & 20 & 600 \\
\hline $\operatorname{ran} 10 \times 12$ & 240 & 120 & 0 & 120 & 143 & 120 & 22 & 720 \\
\hline $\operatorname{ran} 10 \times 26$ & 520 & 260 & 0 & 260 & 297 & 260 & 36 & 1560 \\
\hline $\operatorname{ran} 12 \times 12$ & 288 & 144 & 0 & 144 & 169 & 144 & 24 & 864 \\
\hline $\operatorname{ran} 12 \times 21$ & 504 & 252 & 0 & 252 & 286 & 252 & 33 & 1512 \\
\hline $\operatorname{ran} 13 \times 13$ & 338 & 169 & 0 & 169 & 196 & 169 & 26 & 1014 \\
\hline $\operatorname{ran} 14 \times 18$ & 504 & 252 & 0 & 252 & 285 & 252 & 32 & 1512 \\
\hline $\operatorname{ran} 14 x 18 \_1$ & 504 & 252 & 0 & 252 & 285 & 252 & 32 & 1512 \\
\hline ran14x18.disj-8 & 504 & 252 & 0 & 252 & 448 & 415 & 32 & 10781 \\
\hline $\operatorname{ran} 16 \times 16$ & 512 & 256 & 0 & 256 & 289 & 256 & 32 & 1536 \\
\hline $\operatorname{ran} 17 \times 17$ & 578 & 289 & 0 & 289 & 324 & 289 & 34 & 1734 \\
\hline $\operatorname{ran} 4 \times 64$ & 512 & 256 & 0 & 256 & 325 & 256 & 68 & 1536 \\
\hline $\operatorname{ran} 6 \times 43$ & 516 & 258 & 0 & 258 & 308 & 258 & 49 & 1548 \\
\hline $\operatorname{ran} 8 \times 32$ & 512 & 256 & 0 & 256 & 297 & 256 & 40 & 1536 \\
\hline rd-rplusc-21 & 622 & 165 & 0 & 457 & 125900 & 125762 & 137 & 852386 \\
\hline rentacar & 9557 & 9502 & 0 & 55 & 6804 & 511 & 6292 & 42019 \\
\hline rgn & 180 & 80 & 0 & 100 & 25 & 4 & 20 & 540 \\
\hline rlp1 & 461 & 11 & 0 & 450 & 69 & 58 & 10 & 837 \\
\hline rlp2 & 451 & 1 & 0 & 450 & 69 & 58 & 10 & 5396 \\
\hline roll3000 & 1166 & 428 & 492 & 246 & 2296 & 2121 & 174 & 29387 \\
\hline rout & 556 & 241 & 15 & 300 & 292 & 260 & 31 & 2432 \\
\hline
\end{tabular}


Table A.1 - continued from previous page

\begin{tabular}{|l|r|r|r|r|r|r|r|r|}
\hline Name & Cols & Real & Int & Bin & Rows & Ineq & Eq & NZ \\
\hline roy & 149 & 99 & 0 & 50 & 163 & 153 & 9 & 501 \\
set1al & 712 & 472 & 0 & 240 & 493 & 252 & 240 & 1884 \\
set1ch & 712 & 472 & 0 & 240 & 493 & 252 & 240 & 1884 \\
set1cl & 712 & 472 & 0 & 240 & 493 & 252 & 240 & 1884 \\
seymour & 1372 & 0 & 0 & 1372 & 4945 & 4944 & 0 & 34921 \\
seymour.disj-10 & 1209 & 0 & 0 & 1209 & 5109 & 5108 & 0 & 65913 \\
sp97ar & 14101 & 0 & 14101 & 0 & 1762 & 1761 & 0 & 305069 \\
sp97ic & 1662 & 0 & 944 & 718 & 2087 & 2086 & 0 & 68294 \\
sp98ar & 5478 & 0 & 2682 & 2796 & 4681 & 4680 & 0 & 237234 \\
sp98ic & 2508 & 0 & 1369 & 1139 & 2312 & 2311 & 0 & 140561 \\
sp98ir & 1680 & 0 & 809 & 871 & 1532 & 1531 & 0 & 73384 \\
stein15 & 15 & 0 & 0 & 15 & 37 & 36 & 0 & 135 \\
stein27 & 27 & 0 & 0 & 27 & 119 & 118 & 0 & 405 \\
stein45 & 45 & 0 & 0 & 45 & 332 & 331 & 0 & 1079 \\
stein9 & 9 & 0 & 0 & 9 & 14 & 13 & 0 & 54 \\
stp3d & 204880 & 0 & 0 & 204880 & 159489 & 131403 & 28085 & 737776 \\
swath & 6805 & 81 & 0 & 6724 & 885 & 380 & 504 & 34966 \\
t1717 & 73885 & 0 & 0 & 73885 & 552 & 0 & 551 & 399574 \\
Test3 & 72215 & 39072 & 25969 & 7174 & 50681 & 50083 & 597 & 617907 \\
timtab1 & 397 & 226 & 107 & 64 & 172 & 0 & 171 & 957 \\
timtab2 & 675 & 381 & 181 & 113 & 295 & 0 & 294 & 1731 \\
tr12-30 & 1080 & 720 & 0 & 360 & 751 & 390 & 360 & 3228 \\
vpm1 & 378 & 210 & 0 & 168 & 235 & 192 & 42 & 917 \\
vpm2 & 378 & 210 & 0 & 168 & 235 & 192 & 42 & 1085 \\
\hline \hline
\end{tabular}


Table A.2: List of MIP instances taken from MIPLIB 2003, MIPLIB 3.0 and MIPLIB 2.0. These MIP instances are used in the experiments described in Sections 4.1.2, 6.5.1, and 6.5.2.

\begin{tabular}{|l|l|l|l|l|}
\hline 10teams & dcmulti & markshare1 & mzzv42z & rgn \\
a1c1s1 & disctom & markshare2 & net12 & roll3000 \\
aflow30a & ds & mas284 & noswot & rout \\
aflow40b & dsbmip & mas74 & nsrand-ipx & set1al \\
air01 & egout & mas76 & nw04 & set1ch \\
air02 & enigma & misc01 & opt1217 & set1cl \\
air03 & fast0507 & misc02 & p0033 & seymour \\
air04 & fiber & misc03 & p0040 & sp97ar \\
air05 & fixnet3 & misc04 & p0201 & stein15 \\
air06 & fixnet4 & misc05 & p0282 & stein27 \\
arki001 & fixnet6 & misc06 & p0291 & stein45 \\
atlanta-ip & flugp1 & misc07 & p0548 & stein9 \\
bal8x12 & gen & mitre & p2756 & stp3d \\
bell3b & gesa2 & mkc & pipex & swath \\
bell4 & gesa2-o & mod008 & pk1 & t1717 \\
bell5 & glass4 & mod010 & pp08a & timtab1 \\
bienst1 & gr4x6 & mod011 & pp08aCUTS & timtab2 \\
bk4x3 & harp2 & mod013 & protfold & tr12-30 \\
blend2 & khb05250 & modglob & qiu & vpm1 \\
bm23 & l152lav & momentum1 & ran10x10a & vpm2 \\
cap6000 & liu & momentum2 & ran10x10b & \\
cracpb1 & lp41 & momentum3 & ran10x12 & \\
dano3mip & lseu & msc98-ip & rd-rplusc-21 & \\
danoint & manna81 & mzzv11 & rentacar & \\
\hline
\end{tabular}


Table A.3: List of MIP instances in Table A.1 which are solved within 30 minutes for the experiment in Section 7.1 using the default GLPK node selection configuration, DEBP-NONA-NOAN.

\begin{tabular}{|l|l|l|l|l|}
\hline 22433 & enigma & misc04 & neos-807639 & ran10x10a \\
23588 & fiber & misc05 & neos-807705 & ran10x10b \\
10teams & fixnet4 & misc06 & neos-810286 & ran10x12 \\
air06 & fixnet6 & misc07 & nug08 & ran4x64 \\
bal8x12 & flugpl & mod008 & nw04 & ran6x43 \\
bell3b & gen & mod013 & p0033 & rentacar \\
bienst1 & gr4x6 & neos-506422 & p0201 & rgn \\
blend2 & gt2 & neos-584851 & p0282 & roy \\
bm23 & irp & neos-709469 & p0291 & set1al \\
dano3_3 & khb05250 & neos-777800 & p0548 & stein15 \\
dano3_4 & l152lav & neos-785899 & p2756 & stein27 \\
dano3_5 & lseu & neos-785914 & pipex & stein45 \\
dcmulti & mas284 & neos-801834 & pp08a & stein9 \\
dsbmip & misc01 & neos-803219 & qap10 & Test3 \\
egout & misc02 & neos-803220 & qnet1 & vpm1 \\
eilD76 & misc03 & neos-806323 & qnet1_o & \\
\hline
\end{tabular}


Table A.4: List of MIP instances in Table A.1 which are solved within 60 minutes for the experiment in Section 7.2 using at least one of the node selection configurations listed in Table 7.9.

\begin{tabular}{|l|l|l|l|l|}
\hline 22433 & fiber & neos-480878 & neos-803220 & qnet1_o \\
23588 & fixnet4 & neos-501453 & neos-806323 & ran10x10a \\
10teams & fixnet6 & neos-503737 & neos-807639 & ran10x10b \\
air06 & flugpl & neos-506422 & neos-807705 & ran10x10c \\
aligninq & gen & neos-512201 & neos-810286 & ran10x12 \\
bal8x12 & gr4x6 & neos-522351 & neos-810326 & ran10x26 \\
bc1 & gt2 & neos-530627 & neos11 & ran17x17 \\
bell3b & irp & neos-555694 & neos2 & ran4x64 \\
bell4 & khb05250 & neos-565815 & nug08 & ran6x43 \\
bienst1 & l152lav & neos-570431 & nw04 & ran8x32 \\
blend2 & lseu & neos-584851 & p0033 & rentacar \\
bm23 & mas284 & neos-612125 & p0201 & rgn \\
cap6000 & mas76 & neos-655508 & p0282 & rlp2 \\
dano3_3 & misc01 & neos-686190 & p0291 & roy \\
dano3_4 & misc02 & neos-709469 & p0548 & set1al \\
dano3_5 & misc03 & neos-777800 & p2756 & stein15 \\
dcmulti & misc04 & neos-780889 & pipex & stein27 \\
disctom & misc05 & neos-785899 & pk1 & stein45 \\
dsbmip & misc06 & neos-785914 & pp08a & stein9 \\
egout & misc07 & neos-796608 & prod1 & Test3 \\
eilD76 & mod008 & neos-801834 & qap10 & vpm1 \\
enigma & mod013 & neos-803219 & qnet1 & \\
\hline
\end{tabular}


Table A.5: List of MIP instances in Table A.1 which are not solved within 60 minutes for the experiment in Section 7.2 using any of the node selection configurations listed in Table 7.9. MIP instances for which no incumbent solution was found using any of these configurations are excluded.

\begin{tabular}{|l|l|l|l|l|}
\hline a1c1s1 & mod011 & neos-504815 & neos-631694 & nsrand-ipx \\
aflow30a & modglob & neos-520729 & neos-631784 & p6b \\
aflow40b & mzzv11 & neos-525149 & neos-641591 & pg \\
air04 & mzzv42z & neos-538867 & neos-662469 & pg5_34 \\
air05 & n3700 & neos-538916 & neos-691058 & pp08aCUTS \\
bc & n3701 & neos-544324 & neos-775946 & prod2 \\
bell5 & n3702 & neos-547911 & neos-787933 & protfold \\
bienst2 & n3703 & neos-548047 & neos-791021 & qiu \\
binkar10_1 & n3704 & neos-548251 & neos-799838 & ran12x12 \\
d10200 & n3705 & neos-551991 & neos-808072 & ran12x21 \\
d20200 & n3706 & neos-555298 & neos1 & ran13x13 \\
dano3mip & n3707 & neos-555424 & neos10 & ran14x18 \\
danoint & n3708 & neos-555771 & neos12 & ran14x18_1 \\
ds & n3709 & neos-555927 & neos13 & ran14x18.disj-8 \\
fast0507 & n370a & neos-574665 & neos14 & ran16x16 \\
germanrr & n370b & neos-582605 & neos15 & rlp1 \\
gesa2-o & n370c & neos-584146 & neos17 & roll3000 \\
haprp & n370d & neos-584866 & neos18 & rout \\
liu & n370e & neos-595905 & neos3 & set1ch \\
markshare1 & neos-430149 & neos-598183 & neos5 & seymour \\
markshare2 & neos-476283 & neos-603073 & neos6 & seymour.disj-10 \\
mas74 & neos-494568 & neos-611135 & neos7 & t1717 \\
mcf2 & neos-495307 & neos-611838 & neos9 & timtab1 \\
mcsched & neos-498623 & neos-612143 & newdano & timtab2 \\
mkc & neos-501474 & neos-612162 & noswot & tr12-30 \\
mkc1 & neos-504674 & neos-631164 & nsa & vpm2 \\
\hline
\end{tabular}


Table A.6: List of MIP instances in Table A.1 which are not solved and no incumbent solution was found within 60 minutes for the experiment in Section 7.2 using any of the node selection configurations listed in Table 7.9.

\begin{tabular}{|l|l|l|}
\hline atlanta-ip & neos-565672 & neos-807456 \\
fiball & neos-578379 & neos-808214 \\
gesa2 & neos-583731 & neos16 \\
momentum1 & neos-585467 & neos19 \\
momentum3 & neos-595904 & neos20 \\
msc98-ip & neos-595925 & neos4 \\
nag & neos-619167 & npmv07 \\
neos-506428 & neos-631709 & ramos3 \\
neos-555001 & neos-785912 & rd-rplusc-21 \\
neos-555343 & neos-799711 & sp98ic \\
neos-555884 & neos-807454 & stp3d \\
\hline
\end{tabular}




\section{Appendix B}

\section{Experimental Data}

\section{B.1 Branching Variable Selection Data}

This appendix shows the measurements described below for the branching variable selection methods experiment described in Section 6.5.1.

Name: The name of a MIP instance.

Time: The total time in seconds required to solve a MIP; -1 if a MIP is not solved due to solver error.

Iters: The total number of simplex iterations required to solve a MIP; -1 if a MIP is not solved due to solver error.

Nodes: The total number of branch and bound tree nodes solved; -1 if a MIP is not solved due to solver error.

Best: The last incumbent solution found; "not" if no incumbent solution is found; -1 if a MIP is not solved due to solver error.

Solved: Whether or not a MIP is solved: 0 for not solved, 1 for solved.

The tables of full results can be found at http://www.sce.carleton.ca/faculty /chinneck/students/Wojtaszek/branching_variable_selection_data.pdf. 


\section{B.2 Cuts Data}

This appendix shows the measurements described in Appendix B.1 for the root node cuts experiment described in Section 6.5.2.

The tables of full results can be found at http://www.sce.carleton.ca/faculty /chinneck/students/Wojtaszek/cuts_data.pdf.

\section{B.3 Identification of Promising Configurations Data}

This appendix shows the measurements described in Appendix B.1 for the MIP instances solved in the experiment comparing all of the configurations of backtracking node selection methods, backtrack triggering methods, and active node search thresholds, described in Section 7.1. Only small MIP instances are used due to the excessive amount of time that would be required if all MIP instances were used. The MIP instances used in this experiment are those taken from Table A.1 that are solved using default GLPK within 30 minutes excluding any instances that are MIP feasible at the root node. This set of MIP instances is shown in Table A.3.

The tables of full results can be found at http://www.sce.carleton.ca/faculty /chinneck/students/Wojtaszek/identification_promising_configurations_data.pdf.

\section{B.4 Testing of Promising Configurations Data}

This appendix shows the measurements for the MIP instances solved in the experiment described in Section 7.2 comparing the proposed configurations of backtracking and aspiration node selection methods with the state of the art. These measurements are described in Appendix B.1. Another measure used in this experiment is the proveable optimality gap (see Definition 2), Ogap, at the termination of the branch and bound method. The MIP instances used in this experiment are those taken from Table A.1 excluding any instances that are MIP feasible at the root node. 
The tables of full results can be found at http://www.sce.carleton.ca/faculty /chinneck/students/Wojtaszek/testing_promising_configurations_data.pdf.

\section{B.5 Proof of Concept: Perfect Aspiration Value Data}

This appendix shows the measurements described in Appendix B.1 for the proof of concept experiment described in Section 4.1.2.

The tables of full results can be found at http://www.sce.carleton.ca/faculty /chinneck/students/Wojtaszek/proof_of_concept_data.pdf. 